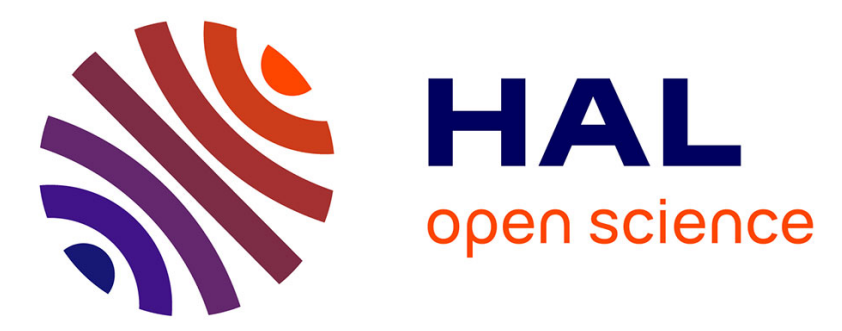

\title{
Le Mané Lud en images : interprétations de signes gravés sur les parois de la tombe à couloir néolithique de Locmariaquer (Morbihan) \\ Serge Cassen
}

\section{> To cite this version:}

Serge Cassen. Le Mané Lud en images: interprétations de signes gravés sur les parois de la tombe à couloir néolithique de Locmariaquer (Morbihan). Gallia Préhistoire - Archéologie de la France préhistorique, 2007, 49, pp.197-258. 10.3406/galip.2007.2455 . hal-02345835

\section{HAL Id: hal-02345835 \\ https://hal.science/hal-02345835}

Submitted on 16 Jan 2020

HAL is a multi-disciplinary open access archive for the deposit and dissemination of scientific research documents, whether they are published or not. The documents may come from teaching and research institutions in France or abroad, or from public or private research centers.
L'archive ouverte pluridisciplinaire HAL, est destinée au dépôt et à la diffusion de documents scientifiques de niveau recherche, publiés ou non, émanant des établissements d'enseignement et de recherche français ou étrangers, des laboratoires publics ou privés.

\section{(이) $\$$}

Distributed under a Creative Commons Attribution - NonCommercial - NoDerivatives| 4.0 


\title{
LE MANÉ LUD EN IMAGES
}

\author{
Interprétations de signes gravés sur les parois \\ de la tombe à couloir néolithique de Locmariaquer (Morbihan)
}

\author{
Serge CASSEN*
}

\begin{abstract}
Mots-clés. Stèle, archéologie des images, pouvoir des images.
Résumé. Le "grand tumulus " du Mané Lud est essentiellement connu pour sa tombe à couloir - d'adjonction secondaire -, qui en borne l'extrémité occidentale et qui contient un nombre significatif de gravures comptant parmi les plus célèbres du corpus européen. Dans un article antérieur, dix orthostates ont été décrits et illustrés par des photographies et des dessins. Seront ici rappelés la nouvelle acquisition technique des images, les modes de représentations des signes et des stèles, et la nature des tracés.

Le corpus des signes est ensuite décomposé et l'interprétation renouvelée. La Grande Déesse devient un cachalot soufflant, l'herminette est un homme aux bras en croix, la hache du bûcheron est une arme guerrière, la houlette du berger n'est qu'un boomerang, le cornu devient un oiseau volant, le peigne-graffiti est un bateau monté, liidole en écusson rassemble un monde en miniature.

Cet article plaide pour une archéologie des images, catégorie malmenée au sein de laquelle image perçue et image créée sont en perpétuelle concurrence mais aussi en continuelle confusion dans l'œuvre d'une archéologie commune de la Préhistoire, et dans ce qu'elle nomme art pariétal ou rupestre ou rock art.

Les gravures du Mané Lud sont les signes de la Miniature et de l'Immensité. Mais au-delà de ce rapport phénoménologique ici exposé, c'est toute la question des images du pouvoir qui est donnée à entendre, souvent limitée aux problèmes historiques sous-tendus par l'écriture. Et sill peut sembler vain de vouloir chercher dans l'écriture en tant que telle, ou dans cette suite de signes gravés, l'origine de toute hiérarchie ou de toute domination, on pourra néanmoins s'interroger sur le rôle des agencements de signes gravés " alignés » sur l'écran minéral, dans la transformation du savoir politique, dans la mise en place de nouveaux modes de pensée correspondant à de nouveaux modes de domination que reflète à nos yeux l'érection, en un temps si court, d'ouvrages parmi les plus gigantesques du $V$ millénaire européen. Car les premiers systèmes d'écriture, et même ces procédés graphiques plus anciens qui jalonnent l'histoire gravée d'un commencement, ont influencé à la fois l'organisation de la vie sociale et celle des systèmes de connaissance.

Voici la prouesse technique poussée par la raison symbolique : des centaines de tonnes de pierres transférées en bord d'océan, en limite de l'inconnaissable, pour servir de miroir mémorable au mythe, peut-être celui d'une origine, de la fondation d'un roi. Et quand bien même l'on ne peut pas réduire un message au moyen matériel de sa transmission, tout changement dans le système des communications - et la «culture " archéologique est aussi cet enchaînement d'actes de communication - a nécessairement en retour d'importants effets sur les contenus transmis.
\end{abstract}

Ceci est le Néolithique nouveau en sa révolution occidentale.

Keywords. Stele, archaeology of pictures, power of pictures.

Abstract. The "Grand Tumulus » of the Mané Lud is primarily known for its passage grave - of secondary addition - which borders its western extrimity and contains a significative number of engravings that are among the most well know in Europe. In a previous article, ten orthostates were described and illustrated with photos and drawings. In this paper, new techniques of recording the images are describe, along with the modes of representation of the signs and steles and the nature of the traces.

The corpus of sign is then decomposed and the interpretation renewed. The Great Goddess becomes a spooting whale; the adze a man with outstretched arms; the woodcutter's ax a weapon of war, the shepherd's crook only a boomerang; the horn sign a flying bird; the brush-graffiti a mounted boat; the escutcheon idol a world in miniature.

In this paper, we plead for an archaeology of pictures. A poorly manipulated category in which the percieved image and the created image are in perpetual competition but also in continual confusion in the works of prehistoric archaeology, and in that which it names parietal art or rock art.

* UMR 6566 du CNRS, Laboratoire de préhistoire et protohistoire de l'ouest de la France, Université de Nantes, BP 81227 , F-44312 Nantes Cedex 3. Courriel : serge.cassen@univ-nantes.fr 
The engravings of Mané Lud are signs of the Miniature and the Immensity. But beyond the phenomenological relation exposed, it is images of power that are in question here, which are often limited to historic problems underlain by writing. And though it may seem vain to search within writing itself, or in this suite of engraved signs, the origin of all hierarchy or domination, we can nonetheless question the role of the arrangements of engraved signs "aligned" on a mineral screen, in the transformation of political knowledge, and in the establishment of new manners of thinking, which correspond to new modes of domination, which reflect for us the rapid erection of some of the most gigantic in $5^{\text {th }}$ millennium Europe. Because these first writing systems, and even these most ancient graphic processes, which mark beginning of the history of engraving, influenced both the organization of the social life and that of the systems of knowledge. Here we see the technical feat pushed by symbolic reason, hundreds of tons of stones transferred to the edge of the ocean, to the limit of the unknowable, to serve as a memorable mirror to myth, perhaps that of an origin, of the foundation of a king. And even though we cannot reduce a message to its material means of transmission, any change in a system of communications - and archaeological "culture" is also a series of acts of communication - forcibly changes the meaning of the contents transmitted.

This is the new Neolithic in its western revolution.

Schlüsselwörter. Grabsäule, Archäologie der Bilder, Gewalt der Bilder.

Translation : Magen O'FARRELL

Zusammenfassung. Der „große Tumulus“ des Mané Lud verdankt seine Berühmtheit vor allem seinem - erst später hinzugefügten Ganggrab, das ihn im Westen begrenzt und eine große Anzahl von Gravuren aufweist, die zu den berühmtesten des europäischen Corpus zählen. In einem früheren Artikel wurden zehn Orthostaten beschrieben und fotografisch sowie zeichnerisch dargestellt. Hier werden nun die digitale Bilderfassung, die Darstellungsweisen der Zeichen und der Stelen und die Natur der Linien wiederaufgenommen.

Das Corpus der Zeichen wird anschließend analysiert und neu interpretiert. Die Große Göttin wird ein blasender Pottwal; die Dechsel ist ein Mann mit gekreuzten Armen, das Beil des Holzfällers eine Kriegswaffe, der Hirtenstab nur ein Bumerang, das hornförmige Zeichen ein fliegender Vogel, das Graffito bzw. der Kamm ein besetztes Boot und die „Muttergöttin“ stellt die Welt im Kleinen dar.

In diesem Artikel plädieren wir faktisch für eine Archäologie der Bilder, eine vernachlässigte Kategorie, bei der das wahrgenommene und das geschaffene Bild nicht nur in fortwährendem Wettbewerb miteinander stehen, sondern in der Archäologie der Vorgeschichte überhaupt und in dem was sie Fels- oder Höhlenmalerei oder rock art nennt, auch fortwährend miteinander verwechselt werden.

Die Gravuren des Mané Lud sind die Zeichen des Kleinen und des unermesslich Großen. Doch über den hier vorgelegten phänomenologischen Bericht hinaus stellt sich im weiteren Sinne die Frage nach der bildlichen Darstellung der Macht, die sich oft auf historische Probleme beschränkt, die mehr oder weniger auf der Schrift basieren. Und wenn es auch zwecklos erscheinen mag, in der Schrift als solcher oder in dieser Abfolge eingravierter Zeichen den Ursprung jeder Hierarchie oder jeder Herrschaft suchen zu wollen, so ist man doch berechtigt sich die Frage zu stellen, welche Rolle diese in Stein eingravierten „aneinander gereihten“ Zeichen gespielt haben mögen bei der Wandlung des politischen Bewusstseins, bei der Einführung neuer Denkweisen, die neuen Herrschaftsweisen entsprechen, die in unseren Augen das Erstellen in einer so kurzen Zeit von Monumenten reflektiert, die zu den gigantischsten des 5. Jahrtausends in Europa gehören. Denn die ersten Schriftsysteme und selbst diese noch älteren graphischen Darstellungsweisen, welche die eingravierte Geschichte eines Anfangs kennzeichnen, haben zugleich die Organisation des sozialen Lebens beeinflusst und die Erkenntnissysteme bereichert.

Dies ist die von symbolischen Motiven getriebene Glanzleistung der Technik: hunderte Tonnen von Felsgestein an die Meeresküsten zu transportieren, an die Grenzen des Unergründlichen, damit sie dem Mythos als unvergesslicher Spiegel dienen, vielleicht dem eines Ursprungs, der Gründung eines Königtums. Und selbst, wenn eine Nachricht nicht auf das Instrument ihrer Übermittlung reduziert werden darf, so wirkt sich doch jede Änderung im Kommunikationssystem - und die archäologische „Kultur“ ist ebenfalls Teil dieser Abfolge von Kommunikationshandlungen - zwangsläufig auf die übermittelten Inhalte aus.

Dies ist das neue Neolithikum in seiner westlichen Revolution.

Übersetzung: Isa ODENHARDT-DONVEZ

\section{L'idée}

«-Qu'est-ce qu'une idée?

- C'est une image qui se peint dans mon cerveau.

- Toutes vos pensées sont donc des images?

- Assurément ; car les idées les plus abstraites ne sont que les filles de tous les objets que j'ai aperçus. »

En un dialogue rarement démenti par notre actualité, ainsi se racontait Voltaire (1764, p. 223) bien avant que son
Dictionnaire philosophique ne soit corrigé, ici sous l'effet d'une dynamique de «l'image imaginée » que portera calmement G. Bachelard, là encore depuis cet autre pôle de réflexion développé par C. Lévi-Strauss, dans lequel règne le signe impérieux qui suit l'image et précède le concept.

Et l'on a beau vouloir suivre Voltaire quand il écrit que toutes les facultés du monde n'empêcheront jamais les philosophes de voir, en effet, que nous commençons par sentir, et que notre mémoire n'est qu'une sensation continuée, 
l'archéologie des images que nous mettons en œuvre nous obligera d'en venir tout d'abord à la capture réfléchie des données, puis à des formes de classements; classements ardus mais les plus bénéfiques à nos enquêtes, en nous gardant, peut-être, pour débuter, d'immédiates sensations nantaises...

Puis, une fois l'enregistrement achevé et le regard déshabitué, cette archéologie nous imposera alors de revenir sur cet irrésistible attrait, sur ces enchantements des formes qui fondent l'étonnant pouvoir des images. Une puissance d'évocation, certes, mais afin qu'émerge une évidence propre à soutenir notre conclusion : voici les images du pouvoir données à entendre sur les stèles morbihannaises, autre objet de nos études.

\section{Le cadre}

On s'accordera, avant cela, sur quelques termes.

L'étude iconologique essaie non seulement de dégager le contenu des images étudiées, mais également de décrire leur morphologie. Ces deux objectifs s'imposeront impérativement dans le travail effectué au sein de la tombe du Mané Lud.

Toute iconographie impliquant un système collectif de communication ou d'expression, nous admettrons agir ensuite au sein d'une série de conventions arbitraires employées par un groupe social aux fins de transmission d'informations et d'expression d'idées et de sentiments.

$\mathrm{Au}$ jeu du catalogage des courants, nous pouvons enfin admettre que nous agissons, en un mot, dans ce Mané Lud en images, au cœur du gros mot d'herméneutique, ensemble de nos connaissances et des techniques qui permettent dans notre discipline de faire parler ces signes gravés et de découvrir leur sens; simplement, plus tard, dans un prochain article: Le ManéLud en mouvement, on insistera sur notre participation à une sémiologie, c'est-à-dire à un ensemble de connaissances et de techniques qui permettent de distinguer où se trouvent les signes, de définir ce qui les institue comme signes, de connaître leurs liens et les lois de leur enchaînement.

Entre les deux courants contraires de l'archéologie contemporaine - d'une part cette exigence de validation empirique afin de neutraliser nos propres biais personnels ou culturels, d'autre part l'école postprocessuelle ralliée aux thèses de l'herméneutique touchant le caractère inévitable des conflits d'interprétation -, il est difficile de conclure que la première l'emporte sur la seconde dans les études relatives à l'art préhistorique ainsi que le prédit J.-C. Gardin, pourtant le meilleur analyste des théories générales et méthodes formelles dans l'interprétation de ces représentations (Gardin, 2000, p. 85), car se dessine un nouveau cadre du travail archéologique au sein des sciences cognitives, une autre voie dans laquelle nos pratiques interprétatives dépendront toujours des contraintes du paradigme logicoscientifique, mais, cette fois ci, probablement aux côtés de la poésie et de la musique...

Parmi les moyens techniques de signification (le langage, les gestes, la représentation plastique et graphique, la technologie et la musique si nous la croyons capable d'exprimer et de communiquer la pensée rationnelle), nous placerons ici le lecteur au cour d'un système de représentation plastique qui entend par ce dernier mot l'ensemble des techniques et des expressions représentatives non graphiques : la peinture, le bas-relief, la gravure, etc., et non la seule technique de la sculpture en rond et les seules expressions que permet cette technique (McLean, 1970, p. 59).

Le Mané Lud en sauvetage a déjà informé sur la technique d'enregistrement et le moyen de vous re-présenter ces formes (Cassen et al., 2005).

\section{Le sujet}

Le monde est notre miniature, écrivait Bachelard (1933, p. 306), car il est si loin, si calme, quand nous le prenons où il est, comme il est, dans le léger dessin de notre rêverie, au seuil de notre pensée. Pour en faire une représentation, pour mettre tous les objets de ces stèles néolithiques à l'échelle, à leur mesure, à leur place véritable, il nous faut briser l'image contemplée dans son unité et il faut ensuite retrouver en soi-même des raisons ou des souvenirs pour réunir et ordonner ce que notre analyse vient de briser. Dialogue de l'esprit et de la matière...

Quand il faudra agir et distinguer, ajoute le philosophe, saisir chaque objet dans sa forme et dans sa force, dans son individualité et son hostilité, notre rêverie se condensera en pensées séparées; l'univers se décomposera ; notre œil, séduit par la main, accommodera. Voici Le Mané Lud en images.

Mais après tant d'efforts séparés, quand il nous faudra classer les valeurs objectives, nous devrons à nouveau éloigner les objets, les réintégrer dans leur atmosphère première, les composer de mieux en mieux par une diminution systématique de l'échelle de représentation. "C'est comme miniature que le Monde peut rester composé sans tomber en morceaux » (id., p. 307). Voici l'annonce du Mané Lud en mouvement. 
Il y aurait donc deux manières de perdre l'univers sensible: ou bien ma rêverie objective s'évaporera tout entière en me laissant glisser dans le rêve proprement dit ; ou bien ma rêverie objective de chercheur se condensera en représentations, et l'univers sensible s'éparpillera en une pluralité d'objets.

L'exigence d'une recherche fondamentale et le gain archéologique attendu passent dès lors par ce passage de la rêverie oisive à la représentation, aux caractères visuels de la représentation, une représentation qui est par essence une systématisation plus vaste, entraînant non seulement la coopération des divers sens, mais encore l'aide de la mémoire et l'organisation de la raison.

\section{LES POUVOIRS DE L'IMAGE}

L'archéologie des images est à entreprendre, catégorie malmenée au sein de laquelle image perçue et image créée sont en perpétuelle concurrence mais aussi en continuelle confusion dans l'œuvre d'une archéologie commune de la Préhistoire, et dans ce qu'elle nomme art pariétal ou rupestre ou rock art.

Si l'on se place réellement en situation imagée, on observe en fait des images qui cherchent leur nom ; la désignation effective est parfois hésitante: alors la Chose (the Thing) existe avant le nom ${ }^{1}$.

Contrairement à l'assertion de Voltaire, G. Bachelard pense que les images ne sont pas des concepts. Elles ne s'isolent pas dans leur signification. Précisément, elles tendent à dépasser leur signification. Images et concepts se forment à ces deux pôles opposés de l'activité psychique que sont l'imagination et la raison (Bachelard, 1960, p. 46).

Dans la pensée scientifique, le concept fonctionne d'autant mieux qu'il est sevré de toute arrière-image. Dès lors, l'image ne peut être étudiée que par l'image, en rêvant les images telles qu'elles s'assemblent dans la rêverie.

Qui plus est, l'imagination n'étant rien d'autre que le sujet transporté dans les choses, les images portent inévitablement la marque du sujet ${ }^{2}$. Et plus faible est l'érudition, plus importante est l'imagination, plus directes sont les images. Et l'archéologue qui croit qu'écrire c'est chercher des images pour illustrer des pensées pose à tort la pensée abstraite avant la métaphore (Bachelard, 1943, p. 182).

1. Voir la déconstruction de The Thing dans la représentation d'un cachalot en Galice (Cassen, Vaquero, 2000).

2. «Et cette marque est si claire que finalement c'est par les images qu'on peut avoir le plus sûr diagnostic des tempéraments. » (Bachelard, 1948, p. 3).
On s'habitue aisément à convenir que c'est la perception des images qui détermine les processus de l'imagination: on verrait les choses d'abord, on les imaginerait ensuite, combinant, par l'imagination, des fragments du réel perçu, des souvenirs du réel vécu, mais on ne saurait atteindre le règne d'une imagination foncièrement créatrice, et tout pont nous reliant au passé serait de fait rompu puisque nous ne pourrions recréer l'image « réelle» d'antan. Mais G. Bachelard réfute cette doctrine et tente d'établir une thèse qui affirme le caractère primitif, le caractère psychiquement fondamental de l'imagination créatrice. Pour lui, l'image perçue et l'image créée sont deux instances psychiques très différentes et il faudrait un mot particulier pour désigner «l'image imaginée »(Bachelard, 1947, p. 3).

Il désigne alors par les rêveries de la volonté ces rêves d'action précise, méconnus parce que la rêverie est toujours considérée sous l'aspect d'une détente. Et de fait, il nous faut redoubler d'attention si nous voulons découvrir l'activité prospective des images, si nous voulons placer l'image en avant même de la perception, comme une aventure de la perception.

L'imagination et la volonté, qui pourraient, dans une vue élémentaire, passer pour antithétiques, sont étroitement solidaires. "On ne veut bien que ce qu'on imagine richement, ce qu'on couvre de beautés projetées » (id., p. 8).

Les images imaginées sont des sublimations des « archétypes » plutôt que des reproductions de la réalité. Aucune image n'est occasionnelle, c'est la perception qui est une occasion. Toute image rendue à sa réalité psychique a une racine profonde; sur l'invite de cette perception occasionnelle, l'imagination revient à ses images fondamentales pourvues, chacune, de leur dynamique propre.

Et pour retrouver les images premières, dans le monde de sensations et de signes où nous vivons et pensons, celles qui expliquent, ensemble, l'univers et l'homme, il faut, ditil, sur chaque objet réanimer de primitives ambivalences, grossir encore la monstruosité des surprises, il faut rapprocher, jusqu'à ce qu'ils se touchent, le mensonge et la vérité (id., p. 183). Voici notre plan de travail au Mané Lud.

Former des images vraiment mutuelles où s'échangent les valeurs imaginaires de la terre et de l'océan, du cristal irisé et de la coquille iridescente, voilà bien, comme on nous l'annonçait, une démarche qui va à l'envers du processus de conceptualisation. Le concept chemine de proche en proche, unissant des formes prudemment voisines ; l'imagination franchit d'extraordinaires différences.

Ajoutons enfin cette autre modulation, plus froide, celle transcrite par C. Lévi-Strauss, pour qui un intermé- 
diaire existe entre image et concept, en l'occurrence le signe, puisqu'on peut toujours le définir à propos de cette catégorie particulière que forment les signes linguistiques, comme un lien entre une image et un concept, qui, dans l'union ainsi réalisée, jouent respectivement les rôles de signifiant et de signifié.

Encore une fois, "l'image " ne peut pas être "idée " (Lévi-Strauss, 1962, p. 61), mais elle peut jouer le rôle de signe, ou, plus exactement, cohabiter avec l'idée dans un signe. L'image est figée, liée de façon univoque à l'acte de conscience qui l'accompagne; mais le signe, et l'image devenue signifiante, s'ils sont encore sans compréhension, c'est-à-dire sans rapports simultanés et théoriquement illimités avec d'autres êtres du même type - ce qui est le privilège du concept -, sont déjà permutables...

Mais cette voie intelligente nous resserre, nous pétrifie dans l'impuissance convenue si l'on se dit esclave du mythe accompagnateur, de paroles lointaines effectivement perdues; aussi nous faut-il revenir à l'archéologie des images, à la recherche des grandes images (Cassen, 1999). Celles qui ont à la fois une histoire et une préhistoire, toujours à la fois souvenir et légende. On ne vit jamais l'image en première instance: toute grande image a un fond onirique insondable et c'est sur ce fond onirique que notre passé personnel met des couleurs particulières. Aussi, c'est très loin dans le cours de la vie qu'on vénère vraiment une image en découvrant ses racines au-delà de l'histoire fixée dans la mémoire. "Dans le règne de l'imagination absolue, on est jeune très tard »(Bachelard, 1957, p. 47).

L'archéologie des images sera ici éclairée par l'image rapide, par l'image instantanée du poète, du peintre, du cinéaste, du photographe, du publiciste, de l'enfant. Quand bien même cette image sera conduite au point d'excès que ne veut point atteindre un esprit raisonnable, alors qu'un excès est pourtant et toujours au sommet d'une image vivante, vivante et non pas achevée et insignifiante.

\section{ENREGISTREMENT ET REPRÉSENTATION DES DONNÉES}

\section{LE TRAVAIL ENTREPRIS}

Le «grand tumulus» du Mané Lud est essentiellement connu pour sa tombe à couloir - d'adjonction secondaire -, qui en borne l'extrémité occidentale et qui contient un nombre significatif de gravures comptant parmi les plus célèbres du corpus européen. Dix orthostates ont été décrits et illustrés par des photographies et des dessins (Cassen et al., 2005). Plusieurs signes relevés se sont d'ailleurs révélés inédits, validant par leur découverte la méthode d'acquisition et de traitement numérique des images mise en ouvre ces dernières années.

Une majorité de gravures est désormais décrite et permet une réinterprétation des signes, ou associations de signes réunies sur un même support, mais également la lecture simultanée de ces orthostates compris comme des monuments à part entière, stèles fonctionnant à l'origine dans un tout autre contexte historique et spatial (fig. 1).

\section{LA RECHERCHE AU PASSÉ ET L'INTERPRÉTATION DES GRAVURES}

Les fouilles menées en 1863 et 1864 par R. Galles et A. Mauricet sont un point de départ reconnu ; une occasion en tout cas de mentionner les gravures et de proposer pour la première fois de voir dans les fameux «signes en $U$ » des jougs d'attelage pour les bovins (Galles, Mauricet, 1864).

L. Davy de Cussé livre en 1865 et 1866 le premier corpus digne de ce nom, ainsi qu'un plan du monument. La stèle de chevet et son énigmatique figure poussent l'auteur à avouer que, malgré le grand relief de la sculpture, la pierre n'a pu être relevée « à cause de l'incertitude des lignes confondues avec les fissures de la pierre ", ce qui est en partie vrai (Davy de Cussé, 1865-1866).

Avec son étude sur les Sculptures lapidaires et signes gravés des dolmens dans le Morbihan, G. de Closmadeuc publie en 1873 un document qui fera date : ses familles de signes, qui ne veulent présumer d'aucune fonction ou identification nominale, regroupent en particulier les «pectiniformes » (en forme de peigne) et les «jugiformes» (en forme de joug) particulièrement présents au Mané Lud.

A. de Mortillet, en 1894, propose de considérer les «pectiniformes » comme autant de dessins de barques; son raisonnement analogique l'ayant porté vers la Scandinavie. Il définit en conséquence, au Mané Lud, des bateaux à proue relevée portant des hommes. Mais il s'avance encore plus loin et conclut que les «jugiformes » sont euxmêmes de simples barques; là encore par analogie avec la Scandinavie, voire avec les pays du pourtour méditerranéen, privant momentanément la Préhistoire armoricaine du signe taurin ramené à ses soi-disant encornures, animal indispensable aux constructions des différentes opinions fondant le contexte agropastoral des sociétés néolithiques, un état socio-économique devant tout naturellement se refléter dans les systèmes de représentation. 
À son tour, G.-H. Luquet conteste l'idée du bateau et, s'il confond comme Mortillet le «jugiforme » et le «pectiniforme » en une seule classe fonctionnelle, c'est pour lui substituer sa théorie de la «ligne frontale »: c'est-à-dire l'ensemble des arcades sourcilières auquel s'adjoint parfois le nez, dans le droit-fil de son interprétation anthropomorphique du signe «scutiforme ". Dans une veine identique, le signe en crosse est, selon lui, issu de la même ligne frontale schématisée (Luquet, 1910). Enfin, et pour la première fois, l'auteur se lance dans la lecture de la pierre de chevet (stèle 1) qui devient la représentation d'un visage "où l'on peut voir, placé au-dessus des yeux, un "jugiforme" se décomposant en deux "pédiformes" " $(i d$.$) . Cette voie qui$ tend vers «l'anthropomorphisme dans l'art ", et singulièrement bornée par toute pierre dressée qui se respecte, se maintiendra désormais sur une ligne ininterrompue jusqu'aux écrits les plus récents touchant l'Armorique ou encore la péninsule Ibérique ${ }^{3}$.

Des fouilles reprennent en 1912 grâce aux activités incessantes de Z. Le Rouzic. Une publication de 1913 lui permet de revenir sur le sujet puisque la «table de dallage couchée » du Mané Lud est à ses yeux un «menhir-statue ».

3. Voir les nombreux travaux, au cours des années 1990, de J. Briard, J. L'Helgouac'h, C.-T. Le Roux, J.-P. Mohen, P. Beneteau, P.-L. Van Berg, N. Cauwe, C. Tilley que l'on trouvera cités en référence dans Cassen, 2000a ; cet anthropomorphisme appliqué et généralisé à la morphologie des pierres est tout aussi vivace en péninsule Ibérique (la plupart des nombreux écrits de P. Bueno Ramirez et R. de Balbín Behrmann, pour la même période et pour les années 2000a ; le point culminant est atteint au Portugal avec les menhirs pseudo-anthropomorphes de l'Alentejo : Varela Gomes, 1997b et à paraître). Le lecteur trouvera un bon parallèle dans le rapport d'Apollon aux pierres brutes qui a fasciné toute une série d'interprètes, convaincus qu'ils pouvaient à la fois atteindre l'étymologie primitive du dieu et saisir le passage de l'aniconique à l'anthropomorphisme (Détienne, 1998, p. 251). Les spécialistes de la cognition ont par ailleurs montré qu'il existe une tendance anthropomorphique relativement aux artefacts et aux représentations visuelles dans de très nombreuses cultures mais également dans notre propre perception visuelle : nous interprétons facilement n'importe quel indice visuel comme une figure humaine (Boyer, 2003, p. 204). Ce penchant pour l'anthropomorphisme serait une conséquence du mode de fonctionnement de notre système cognitif et nous imaginons des agents à forme humaine parce que la personne humaine est plus complexe que les autres types d'objets, et nos processus cognitifs retirent autant d'informations pertinentes que possible de l'environnement afin de produire le maximum d'inférences. Confrontés à des indices ambigus, écrit P. Boyer, nous « voyons » souvent des visages dans les nuages et dans les montagnes... Cette tendance explique aussi que nos dieux et nos esprits aient une forme humaine : même si nous essayons de les décrire autrement, ils sont créés à notre image. En son temps (1954), A. M. Hocart ne voyait dans cette tendance que la vanité grandissante de l'homme... En tout cas, si les dieux et les esprits ne sont pas toujours représentés sous une apparence humaine, ils sont toujours dotés d'un « intellect » humain, ce qui est bien plus spécifique (Boyer, 2003, p. 206).
Ce même chercheur s'associe en 1927 à St.-J. et M. Péquart pour publier le désormais célèbre Corpus des signes gravés des monuments mégalithiques du Morbihan, étude de référence qui, encore aujourd'hui, demeure un ouvrage consulté au même titre qu'un autre inventaire aussi essentiel édité en anglais par E. Shee-Twohig, près de cinquante ans plus tard (1981). Par comparaison avec ses prédécesseurs, le Corpus de 1927 offre, en effet, un meilleur rendu iconographique grâce à un indéniable progrès technique dans la visualisation des gravures (par calque calé et superposé au cliché), sans doute d'un fort coût éditorial mais qui permet de se faire une meilleure appréciation de l'interprétation des figures. Retenons, pour notre propos du moment, que l'acceptation du bateau leur paraît acquise à l'égard des motifs «pectiniformes » morbihannais, et que le signe en U est bien, lui aussi, une forme de transition vers l'embarcation où les passagers (traits verticaux) ont cette fois disparu.

Une mention spéciale doit être portée au crédit du support 21 qui présente des signes composés «en croix », trop proches des représentations humaines de la péninsule Ibérique présentées par $\mathrm{H}$. Breuil à la même époque pour laisser nos collègues indifférents : « les dessins du support en question sont des schémas du corps humain parvenus à un stade de stylisation très poussé » (Péquart et al., 1927, p. 15) ; interprétation qui sera entièrement abandonnée par tous les chercheurs œuvrant après les années 1930, sauf pour l'un d'entre eux (Carenou, 1935, p. 40). Un fait qui mérite d'être souligné puisque les deux exceptions se placent curieusement aux deux extrémités du discours en vigueur à cette époque, la première dans l'ordre du rationnel et du principe de raison, la seconde sous l'emprise d'un envahissement de l'imagination («la mystique dolménique» au service du « Dieu Blé », id., p. 7) à travers de mauvais enregistrements démonétisant aussitôt toute interprétation sur le sujet.

On se souviendra aussi que l'ouvrage magistral de J. Déchelette est le premier lieu d'apparition de l'expression «signe cornu ", précisément à propos des représentations du Mané Lud (1908-1928, p. 612). Cette publication restera la référence fondatrice indépassée pour nommer le signe, compris par analogie avec les représentations mycéniennes. Toute la tradition actuelle en faveur d'une telle reconnaissance trouve sa source dans l'influence souveraine de ce chercheur, et dans la reconduite indiscutée d'une interprétation passée au rang de vérité.

$\mathrm{Au}$ point qu'il faudra directement passer aux écrits des années 1970 pour voir le genre enfin renouvelé. Tout d'abord avec la fouille des tombes à couloir de Colpo (Morbihan) où les signes en U reprennent timidement place du côté de la 

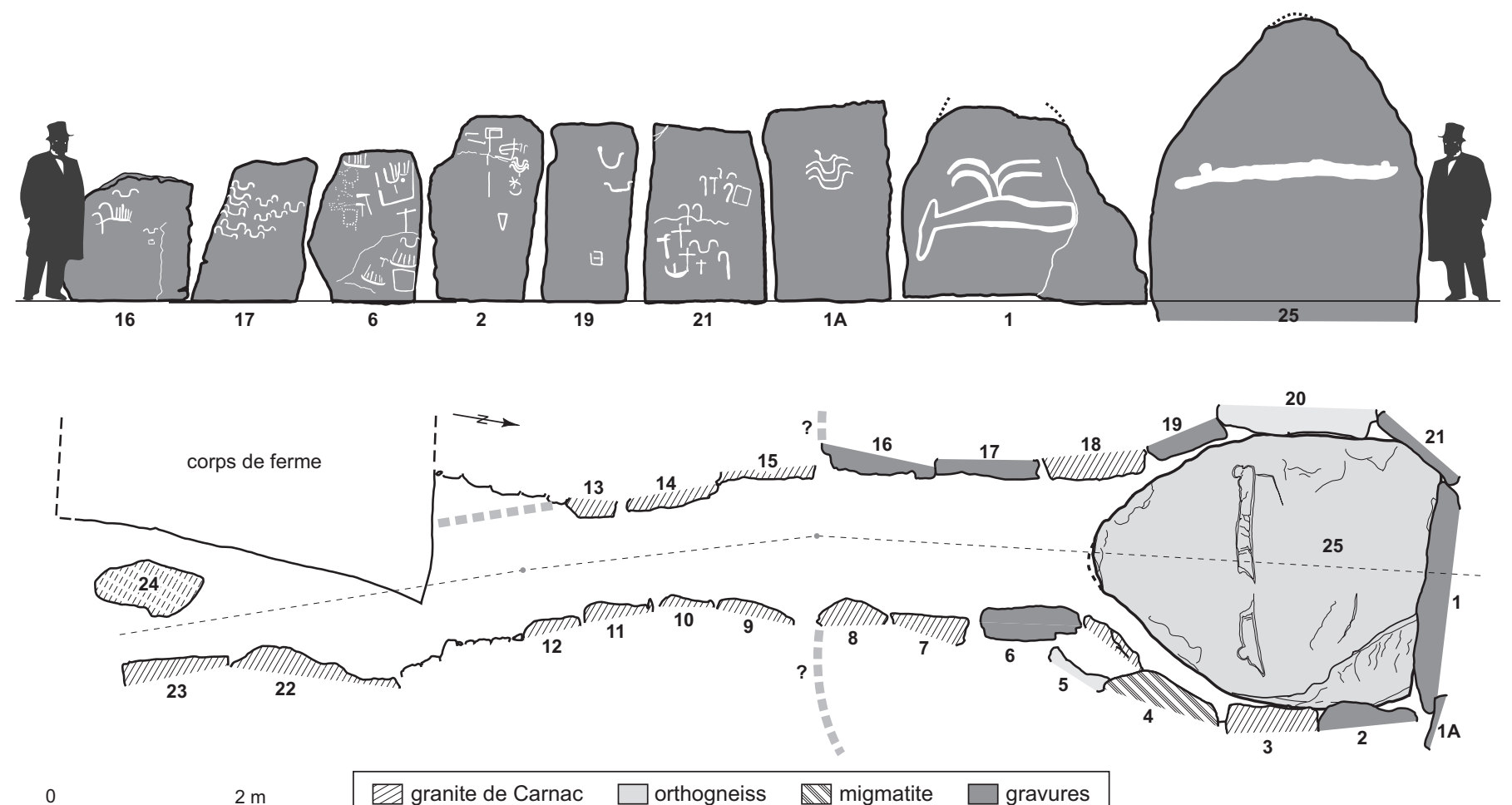

Fig. 1 - Plan de la tombe à couloir du Mané Lud à Locmariaquer, Morbihan (d'après Cassen et al., 2005), et extraction des stèles gravées suivant un ordre croissant arbitraire de leurs tailles.

représentation d'un «visage » (L'Helgouac'h, Lecornec, 1976, p. 394), dans une étonnante continuité avec l'article de Luquet de 1910. Puis c'est la parution de l'ouvrage de E. Shee-Twohig en 1981 qui permet enfin à la communauté scientifique de consulter l'ensemble des représentations, dites mégalithiques, dans un contexte européen et sur un mode graphique clair et homogène. Notre collègue ne prend néanmoins aucun parti et se cantonne à la proposition d'un catalogue purement descriptif.

L'auteur dissocie pourtant le yoke (signe en U « français ») du U motif simple ou à développements concentriques. Le $\mathrm{U}$ motif est en fait une sorte de demi-cercle ou « $\mathrm{U}$ », mais renversé la plupart du temps, qui s'apparente à l'image de «l'arc-en-ciel » tel que nous l'avons reconnu aussi bien dans la thématique céramique castellic que sur les stèles gravées. Quoi qu'il en soit, l'auteur, pour une fois, réfute l'acception fonctionnelle du premier mot (yoke = joug) et ne l'utilise qu'en tant que terme purement descriptif (Shee-Twohig,

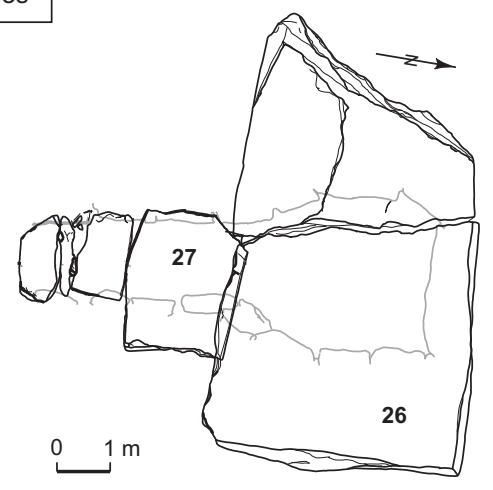

1980, p. 54). On note enfin que la classification proposée dissocie clairement le signe cross (croix) des deux autres signes « hache " et «crosse", ce qui n'est pas sans importance pour l'étude du Mané Lud, alors que les préhistoriens - à l'exception du Corpus des Péquart et Le Rouzic - confondront toujours croix et haches dans une même classe fonctionnelle.

Quant à B. Maisonneuve qui tente courageusement de substituer un vocabulaire neutre aux noms usuels soupçonnés d'être trop connotés par leurs fonctions présupposées, elle partage les gravures du Mané Lud entre "signal $\mathrm{A}$ », « $\mathrm{C}$ », « $\mathrm{D} », ~ « \mathrm{E}$ », rangeant la trop gênante stèle de chevet dans les «signaux singuliers » (Maisonneuve, 1983, p. 50). J. Briard réaffirme le nouveau symbolisme lié aux 
préoccupations des peuples d'agriculteurs et de pasteurs "qui marquent leur vénération pour l'animal élevé par la reproduction de signes cornus»(Briard, 1987, p. 11). Pour sa part, M. Guimbutas confond à nouveau le cornu/ jugiforme et le bateau/pectiniforme au sein d'une même famille de signes, reprenant l'ancienne fusion des termes en une seule et même catégorie de représentation, mais dont la fonction forcée est cette fois destinée, au Mané Lud tout comme au Petit Mont, à Arzon, au seul profit de la fameuse déesse néolithique, divinité régénératrice (Guimbutas, 1989, p. 247).

J. L'Helgouac'h revient sur le Mané Lud en 1994, insistant sur la silhouette humaine qu'il affirme reconnaître dans les contours d'une majorité de stèles, mais laisse encore une fois dans l'ombre la pierre de chevet. La dalle de plancher est ainsi la stèle anthropomorphe où se lisent épaulements et tête. Le spécimen en orthogneiss no 20 est dit «décapité " de son « excroissance sommitale » marquant la tête (L'Helgouac'h, 1994, p. 7). C'est encore la divinité en écusson qui est repérée au bas de la stèle $n^{\circ} 6$ (le quadrilatère), stèle elle-même anthropomorphe où les côtés sont les «épaulements» travaillés. Enfin, le signe cornu est, cette fois, non plus un visage mais la stylisation du symbole taurin.

Le même chercheur revenant sur le Mané Lud, discutant en 1998 de la navigation au Néolithique, n'adopte pas les interprétations antérieures (de Mortillet, puis Péquart et al.) qu'il considère comme «très audacieuses » : «l'identification de ces soi-disant bateaux, [...] reste encore plus sujet à l'imagination ou aux désirs des auteurs » (L'Helgouac'h, 1998, p. 156). Paradoxalement, pour le Néolithique final des tombes allongées à court vestibule («allées-couvertes »), J. L'Helgouac'h reprend l'idée de H. Breuil et M.-E. Boyle (1959, p. 132) selon laquelle la fameuse et énigmatique "palette» serait une rame, une rame qui pourrait avoir été encore mieux un « aviron de gouverne » (L'Helgouac'h, 1998, p. 159) en accord avec la mythologie antique du monde des morts...

Quoi qu'il en soit, ses jugements de valeur sur l'ensemble des signaux, quand ils ne sont pas issus des sites comme ceux de la Table des Marchands ou de Gavrinis «qui atteignent la perfection ", et en dehors de panneaux remarquables, comme celui de Pen Hap, qui montrent la maîtrise de l'exécutant, le poussent à réduire les pseudobateaux du Mané Lud à des "graffitis assez rustiques " par trop décevants (L'Helgouac'h, 1991, p. 544). On se souviendra que H. Breuil, en 1959, était encore plus radical et se posait en observateur en réaction sur son temps à la simple lecture de ces mêmes gravures du Mané Lud et de cet art en Bretagne, si «profondément dégénéré " 4 .

C.-T. Le Roux, en 2001, confirme les diagnostics en usage et se fait, avec J. Briard, le meilleur interprète de cet état d'acceptation généralisé du corniforme, représentant une divinité taurine, probable parèdre masculin de la « déesse à l'écusson », elle-même principale divinité, «vraisemblablement féminine et maîtresse de la fécondité, de la vie et de la mort» (Le Roux, 2001, p. 20). Quant au «grand signe énigmatique » qui se détache en relief sur la dalle de chevet de la chambre du Mané Lud, il faut y voir une possible «stèle en écusson » réemployée dans la construction du monument... Enfin, L. Laporte et C.-T. Le Roux renchérissent en 2004 sur l'absence totale de bateaux sur les parois du monument, et A. Tresset pense pouvoir reconnaître « des cornes de brebis, ou même de chèvre » dans les signes en U, ainsi qu'à travers la classique crosse sur stèle ou sur céramique Castellic (Tresset, 2005, p. 278), ce que J. Markale avait déjà proposé en 1987...

\section{NOUVELLE ACQUISITION DES IMAGES}

Les opérations techniques se sont ici partagées en trois ensembles de tâches :

- levés topographiques classiques par nivellement puis traitement numérique des surfaces et des volumes de la tombe à couloir (autres méthodes: levé tachéométrique par scanning au Mané Rutual ; levé par scanner laser 3D de surface au Mané Kerioned, voir Cassen, Merheb, 2004) ;

- relevés graphiques des orthostates au $1 / 10^{\mathrm{e}}$;

- prises de vues numériques des gravures et traitement infographique en laboratoire.

Les opérations spécifiques portant sur l'enregistrement des signes gravés se sont déroulées comme suit :

- les gravures sont éclairées en lumière artificielle directe mais aussi, parfois, indirecte, voire sous effet tamisé à l'aide d'un parapluie diffuseur ; une seule figure exige en moyenne au moins une vingtaine de clichés pris sous différentes incidences lumineuses;

- le dessin vectoriel appliqué sur cliché est obtenu grâce à un stylet couplé à une tablette graphique ; il suit en règle

4. «[...] aucune logique, aucune anatomie, n'a réglé l'exécution de ces dessins, il y a autant de distance de l'art réaliste des paléolithiques (sic) à l'art super-abstrait, vraiment futuriste et aussi profondément dégénéré, de la plupart des panneaux auxquels je me suis attaqué en Bretagne, qu'on peut voir entre les chefs-d'œuvre de la peinture classique et les aberrations modernes des futuristes les plus abstraits. » (Breuil, Boyle, 1959, p. 3). 
générale la ligne de plus forte surexposition, c'est-à-dire le bord de la gravure directement exposé à la lumière ;

- la synthèse des figures détermine une superposition de traits qui reflètent les deux bords opposés d'un trait en creux, coïncidant plus ou moins nettement selon l'état de conservation de la gravure et selon sa profondeur ;

- un contrôle sur l'état des surfaces, naturelles ou au contraire travaillées (voir l'écrasement des minéraux), est opéré à l'issue des premiers dessins, afin de décider de l'état anthropique ou non de tracés incertains; de nouveaux clichés sont généralement requis avant d'arrêter le choix des dessins retenus ;

- ce choix des tracés est effectué au laboratoire, entre toutes les options relevant du domaine des possibles, de sorte qu'une synthèse graphique est extraite du regroupement le plus significatif (le plus grand nombre de tracés concordants), en confrontant au surplus le résultat obtenu avec les relevés antérieurs ramenés à l'échelle voulue, et seulement à cette étape finale du travail de restitution.

Le redressement de l'image s'établit le plus souvent en aval du processus, quand le dessin vectoriel est superposé aux relevés antérieurs, ou, mieux, à l'estampage produit par nos soins - un estampage qui ne conserve néanmoins de ces tracés aussi altérés que ce que l'opérateur veut bien laisser paraître par la pression de la main dans les creux de la gravure - et aux mesures de points d'ancrage prises directement sur la pierre, points de stabilisation qui peuvent prendre l'aspect concret d'un référentiel géométrique (carré ou rectangle) matérialisé par des boulettes de pâte à modeler appliquées sur la stèle assimilée à un plan. Les erreurs, faibles en photographie numérique, ont été reconnues et spécifiées pour ce site qui impose des prises de vue sans recul.

\section{MODES DE REPRÉSENTATIONS DES SIGNES ET STÈLES}

Suivant l'étape de la synthèse graphique réunissant tous les tracés enregistrés, une proposition de tracé est effectuée pour chaque signe, et soumise au lecteur pour comparaison avec les interprétations données dans la littérature sur le sujet. Une gradation de niveaux de gris peut souligner le degré d'incertitude quand il y a hésitation sur le dessin ; les tracés uniques sont le plus souvent abandonnés.

À la différence du Mané Rutual et du Mané Kerioned où les surfaces des stèles et des orthostates ont fait l'objet de modèles numériques (MNT), les dalles du Mané Lud ont été assimilées à des faces planes sur lesquelles furent inscrites les gravures, seulement rehaussées d'accidents topographiques: arêtes, diaclases, météorisation, etc. Le processus informatique et géométrique d'extrusion a été décrit par ailleurs (Cassen et al., 2005).

\section{NATURE DES TRACÉS}

Les formes sont déterminées par des tracés en creux, sans qu'intervienne au Mané Lud le procédé du champlevé si caractéristique du Mané Rutual voisin ou du plafond de la Table des Marchands; seule la stèle $n^{0} 1$ se rapproche ici de cette technique d'exécution.

Une forme à contour fermé peut conserver cette réserve intérieure intacte, sans enlèvement de matière (stèle $\mathrm{n}^{\circ} 6$; lames de haches). Notre interprétation finale gommera cette distinction et rendra un même effet de remplissage de surface afin d'en mieux définir la figure.

Le tracé peut être continu (le classique trait gravé) ou discontinu, obtenu en ce cas par creusement, semblet-il rotatif, de petites cupules alignées suivant une forme quadrangulaire, curviligne ou rectiligne.

Généralement, le tracé dégagé par percussion posée, offre une section en $U$ de plus en plus ouverte et de moins en moins profonde au fil du temps et de l'érosion de la figure (Mens, 2002). Une exception doit cependant être notée : les traits verticaux des «embarcations " des stèles $n^{\text {os }} 6$ et 16 rendent un effet visuel dissemblable, sous éclairage rasant; ce sont probablement d'autres instruments qui ont présidé à leur réalisation, tracés qui tendent davantage vers une section en $\mathrm{V}$ et plaident en faveur d'une sorte d'incision, si tant est que l'on puisse exprimer une telle action dans le granite de Carnac. Des mesures de profondeur faites à l'aide d'un micromètre de métrologie pourraient valider l'observation visuelle et tactile. Mais la distinction est suffisamment remarquable pour qu'il soit nécessaire de la noter et de pouvoir l'interpréter le moment venu.

\section{LE CORPUS DE SIGNES : DÉCOMPOSITION ET RENOUVELLEMENT}

Si la liste des objets reconnus n'est pas longue au Mané Lud, celle des sujets est plus expansive. La liste des signes ici reproduite a pu être établie en nous appuyant tant sur les ouvrages classiques que sur les plus récentes publications parues sur la matière ${ }^{5}$.

5. À la liste bibliographique que nous proposions dans Cassen, 2000a et $b$, ajoutons quelques références plus récentes : Laporte, Le Roux, 2004 et Joussaume, 2003. 


\section{LA GRANDE DÉESSE OU LE « SIGNAL SINGULIER » : STÈLE $N^{\circ} 1$}

\section{UNE FIGURE DU SILENCE}

Dominant l'absence d'engagement de la part des meilleures signatures au sein de la discipline, une interprétation ("la Grande Divinité ») et une description normative (« la stèle en Écusson ») resteront à ce jour les seules propositions appliquées à la stèle $\mathrm{n}^{\mathrm{O}} 1$, dite de chevet.

De là vient cet espace vacant laissé à la merci des écrits spéculatifs à vocation ésotérique, attirant en Morbihan nombre de curieux sincères mais nostalgiques de brillantes civilisations éteintes et de surcroît détentrices d'un antique savoir perdu, grâce auxquels cette stèle perd son statut très envié de déesse pour devenir une carte marine décrivant, depuis l'Angleterre et l'Irlande, le chemin parcouru par ces habitants de la vieille Europe pour gagner l'Amérique du Nord (Jonge, Wakefield, 2002, p. 7-9).

\section{L'ENREGISTREMENT RENOUVELÉ}

C'est une stèle en granite dit de Carnac (roche claire légèrement feuilletée), plus large que haute dans son implantation actuelle. Le sommet, en partie porteur en tant qu'orthostate dans sa configuration actuelle, a subi de nombreux éclatements, soit au moment de la pose de l'immense dalle de couverture, soit par volonté humaine de calibrer l'arête devant supporter cette dalle.

La gravure n'occupe pas tout l'espace disponible sur la stèle. On peut dès lors supposer que ce décentrage vers la gauche est dû à une utilisation opportuniste du modelé de la surface. Une rupture de pente borde en effet la région droite selon une courbe orientée à la verticale, et l'extrémité de la gravure vient exactement mourir à cet emplacement en surplomb, épousant le point naturel d'inflexion sur la roche.

Soixante-dix photos et dessins (fichiers informatiques) constituent la base du travail sur cette stèle.

\section{LA FORME RECONNUE}

Voici une forme nouvelle, sans doute peu éloignée des restitutions de nos prédécesseurs qui en avaient reproduit les lignes directrices, mais suffisamment distante pour qu'un regard neuf puisse cette fois déclencher un autre processus de reconnaissance, à vrai dire facilité par le réalisme de la figure (fig. 2).
C'est en ce sens qu'une interrogation publique faisant appel à des réponses spontanées fut mise en œuvre en 2003, conformément à nos expériences antérieures menées autour de la Déesse-Mère et du Cornu (Cassen, 2000c et 2005). L'exercice fut tenté sur une promotion de 15 étudiants en DESS de l'université de Nantes («Les métiers de l'archéologie »), sans aucune connaissance préalable du problème, la figure leur étant présentée hors de tout contexte. Voici les réponses répertoriées avec leur accord ${ }^{6}: 12$ ont reconnu une baleine, 2 ont identifié un cachalot et 1 étudiant fut dans l'incapacité de rapporter la gravure à une figuration connue.

De cette unanimité sur le cétacé - qui est le point de vue que nous défendons à propos de la « hache-charrue » dans ce processus de déconstruction du corpus armoricain -, reprenons le droit fil de la reconnaissance.

Les unités graphiques identifiables peuvent être résumées ou décrites comme suit :

- la tête est quadrangulaire ;

- le souffle jaillit en «fontaine », à l'image des figurations familières de l'eau émergente (fig. 2) ;

- la queue est profilée selon une vue latérale, en perspective ;

- la tête et le tronc sont dans un égal rapport de proportions ;

auxquels s'ajoutent :

- la tête "carrée" dégagée par flexion du corps de l'animal ;

- la flexibilité de l'appendice caudal si remarquée en mer lors du plongeon vertical qui laisse alors aisément entrevoir cet affaissement de la queue en une barre perpendiculaire au tronc;

- l'emprise étonnante de la tête qui occupe dans la réalité le tiers du corps de la bête, confirment notre diagnostic premier (Cassen, Vaquero, 2000) : le cétacé n'est pas une baleine, mais bien un cachalot.

Confirmation également, mais inattendue, de la piste ibérique.

Remarquez la manière dont le «souffle » est rejeté vers l'avant, depuis un évent en position anatomique erronée mais suffisamment efficace à l'échelle de la représentation pour rendre compte d'un phénomène étonnant, visuel autant que sonore, qui domine l'animal émergeant au loin et sur lequel cette signature universelle est finalement centrée et abstraite.

6. Les fiches d'interrogation sont consignées dans le Cahier de laboratoire, à la disposition de nos collègues ; nous reviendrons plus loin sur cette enquête. 


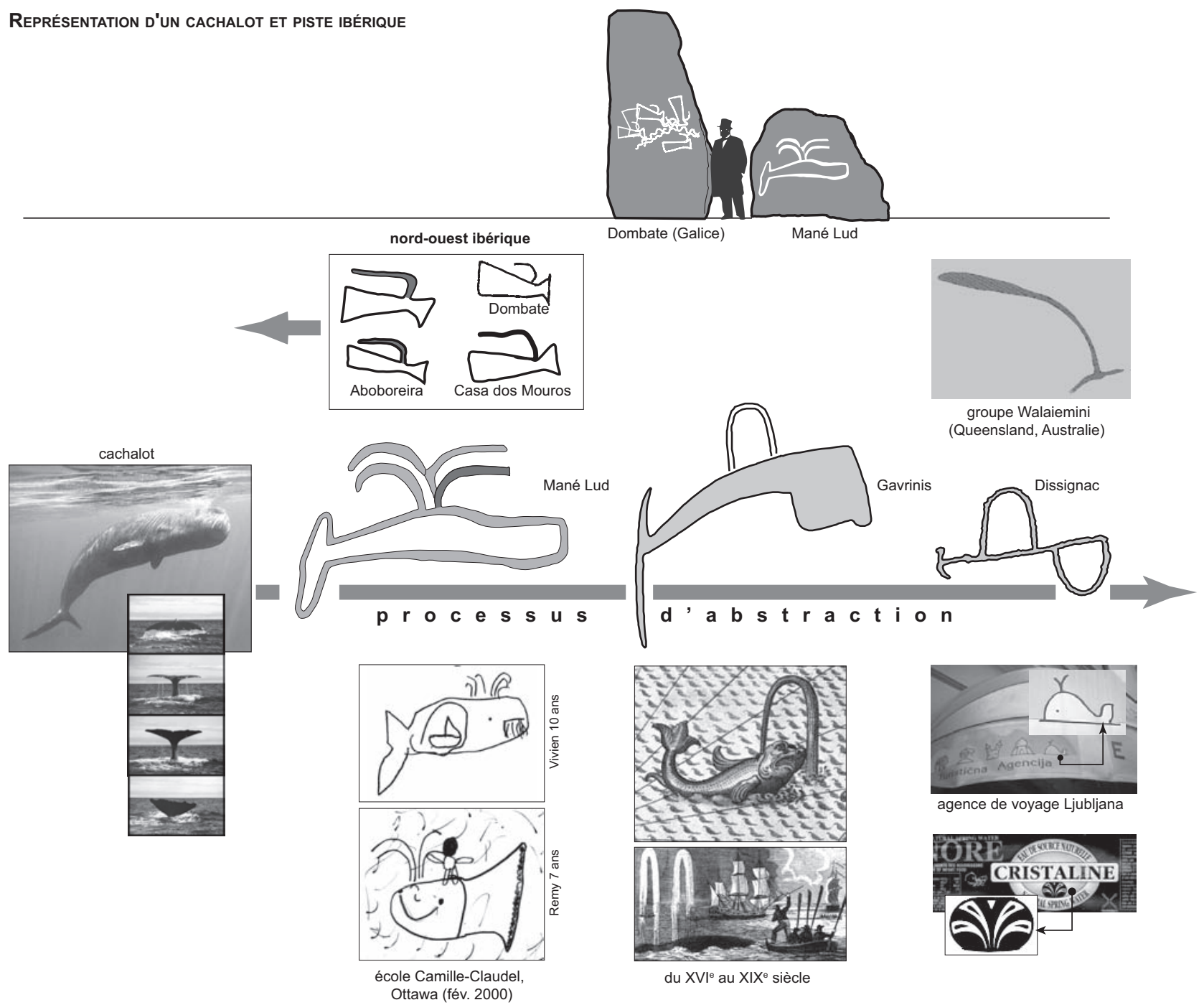

Fig. 2 - Étude graphique sur un processus d'abstraction : la représentation du cachalot en Bretagne et en Galice, convergences sur la forme du jet (d'après Cassen, Vaquero, 2000) ; dessins d'enfants de l'école Camille-Claudel à Ottawa, Canada (réunis par V. Benn-Cassen) ; agence de voyage à Ljubjana, Slovénie (cliché: S. Cassen, CNRS). La gravure de Dissignac à Saint-Nazaire, Loire-Atlantique, a été volontairement inversée, c'est l'unique représentation du corpus armoricain à présenter une tête à gauche.

C'est cette évacuation vers l'avant en une courbe terminée en deux traits rectilignes qui nous autorise à effectuer le rapprochement formel avec la «boucle » dégagée au-dessus de la Chose (the Thing) sur les stèles de Galice et du nord du Portugal (Cassen, Vaquero, 2000, fig. 174).

Une dynamique particulière du tracé, commune aux deux contextes géographiques, qui ne peut procéder d'une simple convergence iconophile mais qui relève au contraire, à la même époque, d'une même idée graphique sous-jacente, d'une même solution partagée/adoptée pour un problème singulier de représentation, confortant l'intuition première reliant l'ouest ibérique et le sud de l'Armorique.

\section{LA HACHE, L'HERMINETTE, LE GRUGIFORME}

Lame de hache isolée et lame emmanchée sont probablement, parmi les signes armoricains, ceux qui furent très tôt reconnus, c'est-à-dire désignés par des noms d'outils immédiatement identifiables par le sens commun et dont le statut instrumental demeurera incontesté jusqu'à nos jours. 


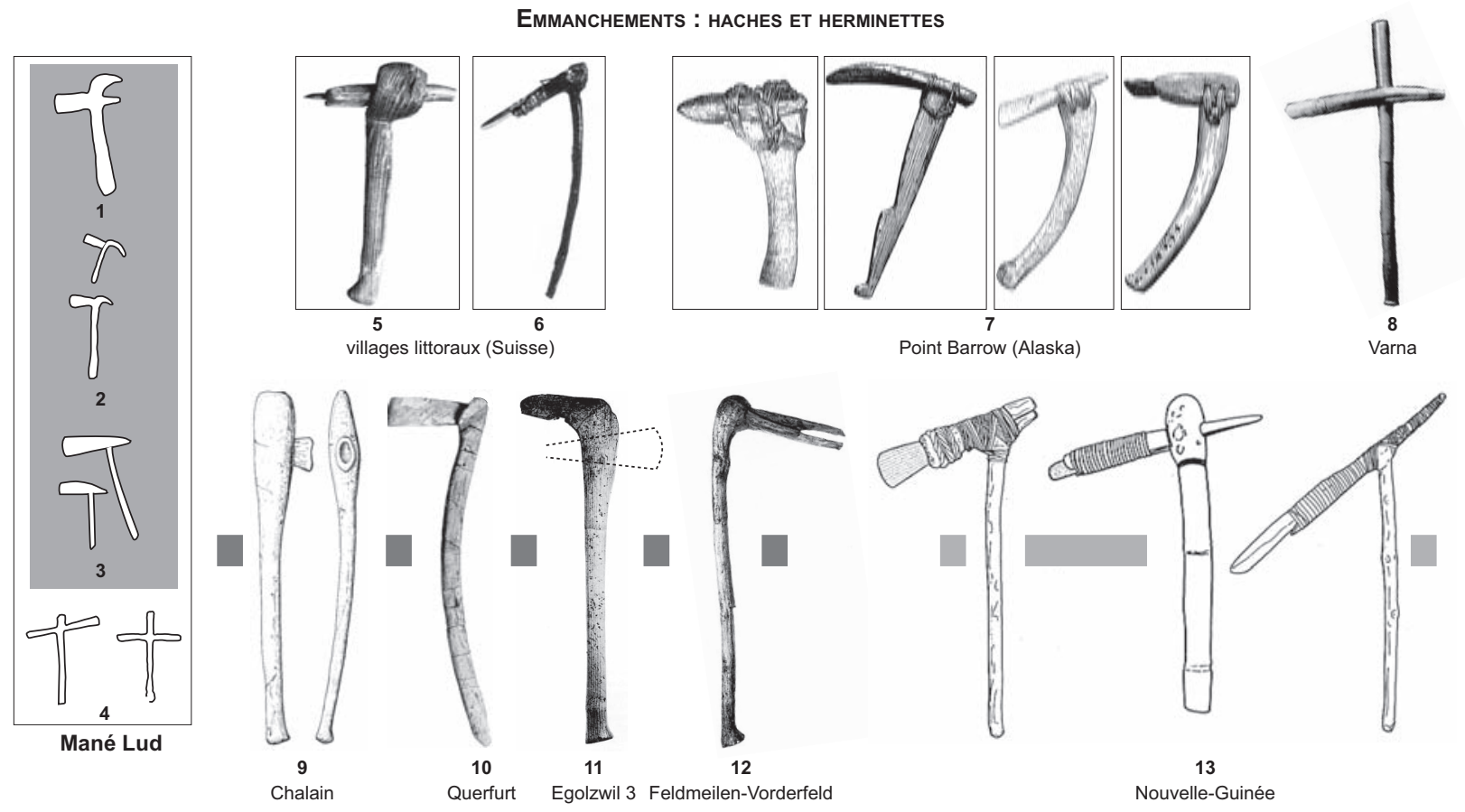

Fig. 3 - Recherche de formes archéologiques similaires aux gravures de haches et cruciformes/herminettes du Mané Lud: herminettes polies emmanchées des villages littoraux de Suisse, fouilles anciennes (d'après Höneisen, 1985); manches longs à mortaise et tête renflée de Chalain à Fontenu, Jura (d'après Baudais, 1985) ; hache directement emmanchée, sans dépassement du talon, de Querfurt à Langeneichstätt, Allemagne (d'après Sprockhoff, 1938) ; manche en crosse de Egolzwil 3, Suisse, et manche coudé avec pince de Feldmeilen-Vorderfeld, Suisse (d'après Höneisen, 1985) ; herminettes provenant de Point Barrow, Alaska (d'après Boas, 1883) ; "hache-sceptre ", pierre et or, tombe $n^{\circ} 4$ de Varna, Bulgarie (d'après Ivanov, 1989); Nouvelle-Guinée, de gauche à droite: hache à pince fixe, hache à tête pivotante et mortaise totale, herminette à pince fixe (d'après Pétrequin, Pétrequin, 1993).

Laissons la fonction symbolique à l'écart et limitonsnous, pour l'instant, à l'analyse des formes.

Trois types d'emmanchements sont décelables sur les stèles du Mané Lud (fig. 3) :

- l'emmanchement direct d'une lame de hache par mortaise creusée dans le bois terminé en crosse, le talon pointu dépassant à l'arrière du manche (fig. $3, \mathrm{n}^{\mathrm{o}} 1$ ) ;

- la fixation d'une lame de hache sur un manche également en forme de crosse, mais cette fois sans qu'aucun talon visible n'outrepasse celui-ci (fig. 3, no 2) ;

- la fixation d'une lame de hache à l'extrémité proximale du manche, sans dépassement de ce dernier ou d'une quelconque volute de crosse, le talon, là encore pointu, dépassant à nouveau la poignée (fig. 3, no 3) ;

- un quatrième type, ou variante simplifiée, est reconnu dans toute la littérature s'intéressant au sujet, mais, nous l'avons dit, à deux exceptions près (Péquart, Le Rouzic, 1927, p. 15 ; Carenou 1935, p. 40). Ce sont les signes que nous désignerons par le terme de cruciformes; généralement interprétés comme des herminettes, ils supposent que la lame, fonctionnant bien entendu de profil pour l'observateur latéral, est alors limitée à un trait perpendiculaire au manche, le talon étant d'une longueur égale à la partie antérieure (fig. 3, n ${ }^{\circ}$ ).

Interrogeons cette dernière interprétation.

\section{Ce signe cruciforme pourrait-il être cette abstraction d'une hache à emmanchement direct?}

Non, car le dessin de ce type précis d'instrument accompagne le cruciforme sur une même stèle (fig. $1, \mathrm{n}^{\circ} 6$ ) ; ici, le tranchant réaliste doit signifier la hache. Une simplification d'un même instrument qui serait juxtaposé à la même échelle et dans la même scène n'est pas défendable, car la technique de réalisation, la composition et le degré d'altération des gravures plaident résolument en faveur de leur contemporanéité. 


\section{Ce signe pourrait-il être alors une représentation d'une herminette à emmanchement direct ?}

Cette interprétation se heurte à quelques difficultés. On ne connaît pas d'exemples ethnographiques rapportant que la lame en pierre serait directement encastrée dans la mortaise creusée, dépassant le manche monoxyle massif d'une longueur identique à la partie «active "; une largeur de mortaise proportionnelle à de telles longueurs d'instruments exigerait, pour les maintenir sans casse, une épaisseur de bois conséquente, épaisseur d'ailleurs inconnue à ce jour sur les spécimens archéologiques.

En revanche, une lame de pierre comprimée transversalement dans une pièce intermédiaire (gaine) de bois ou d'andouiller de cerf propre à amortir les chocs, elle-même introduite dans un manche massif (emmanchement indirect), peut laisser au regard de l'observateur une vague ligne directrice qui pourrait être semblable au cruciforme en question puisque cette pièce intermédiaire est en mesure de dépasser largement la poignée (fig. 3, $\left.\mathrm{n}^{\mathrm{0}} 5\right)$; mais on voit combien cette partie, dans laquelle s'expriment sur l'outil les contraintes majeures, doit être renforcée par un fort noyau de bois qui n'est jamais restitué par la gravure.

Une abstraction poussée sur l'arme-outil laisserait seule la possibilité d'un tel dessin où manche et lame seraient projetés en plan par deux traits se croisant. Mais encore une fois, pourquoi l'herminette à emmanchement indirect serait-elle simplifiée à l'extrême alors que la hache vraie, sur le même support, ne le serait pas?

Par ailleurs, l'archéologie ouest-européenne ne connaît pas de lames d'herminette prévues pour un emmanchement direct où, à l'image de certains spécimens de haches, un anneau non poli serait réservé, laissant apparaître son piquetage pour une meilleure adhérence dans la mortaise du fût monoxyle.

Nous ne connaissons pas non plus d'instruments, haches ou herminettes, qui produiraient une telle symétrie partagée autour de la poignée, une symétrie d'instrument qui ne serait pas un gage d'équilibre puisque trop de poids de la lame en pierre porterait le centre de gravité vers l'arrière.

Un cas exceptionnel pourrait cependant se superposer en partie au dessin du Mané Lud. Les haches-sceptres des tombes $\mathrm{n}^{\text {os }} 4$ et 43 de Varna (Bulgarie) conviendraient, en effet, en tant que modèle de comparaison (fig. 3), avec leur lame polie étroite et allongée, aux bords parallèles (Ivanof, 1989, p. 119 et p. 147) ; la perforation décentrée nous éloigne cependant du cruciforme latin s'il fallait représenter l'arme. Par ailleurs, ces instruments - non fonctionnels, faut-il le préciser, sans compter le dépassement distal exagéré du manche droit rendant la frappe entièrement inadaptée - sont inconnus du corpus des dizaines de milliers d'objets polis de la France du Nord-Ouest, et quand bien même nous rappellerions une ligne graphique similaire avec la grande herminette double (non achevée, en cours de perforation) de la Terre des Pots à Ozillac (Charente-Maritime), un objet lithique dit de prestige dans les tombes du Rubané, probablement objet d'un transfert à longue distance aux débuts du Ve millénaire entre le Bassin parisien et le Poitou-Charentes (Cassen, 2000g, p. 741). Faute de la moindre correspondance régionale et de la stricte identité de forme, ces repères doivent donc être abandonnés.

Les motifs cruciformes du Mané Lud ne sont pas des représentations de haches ou d'herminettes, et cette remise en cause du schéma établi doit valoir pour l'ensemble du corpus armoricain.

En conséquence, toute question à leur sujet doit être différemment posée.

Il faut tout d'abord admettre que le terme cruciforme est bien adapté à l'évocation de la figure. Mais que serait une forme de croix?

La croix, dans la perception immédiate et le nominalisme de ce début de $\mathrm{XXI}^{\mathrm{e}} \mathrm{s}$., renvoie à la religion chrétienne, et particulièrement, pour la forme du Mané Lud, à la croix dite latine de l'Église de Rome.

Cette croix fut le support de la crucifixion de Jésus; elle prend la forme du corps humain, jambes jointes, bras écartés, c'est la projection d'une ligne directrice.

Le signe du Mané Lud est en forme de croix, mais la croix est «adaptée » au corps humain, c'est un objet qui symbolise la Passion, qui identifie enfin une religion et non plus exclusivement le souvenir d'un héros mort.

Cette croix nécessite cependant un développement spécifique en tant que signe galvaudé, un développement rendu indispensable par le fait de son extraordinaire diffusion sur la planète, accompagnant le christianisme et son processus évangélisateur encore vivace aujourd'hui sur tous les continents. Le succès de cette symbolique planétaire est tel que tout cruciforme mal calé dans le domaine archéologique est souvent assigné à une date récente par association de pensée, le corpus gravé armoricain n'y faisant pas exception.

Voici pourtant mention de quelques contextes qui devraient tempérer cette relation unilatérale. 


\section{Motif et thème cruciformes dans l'Europe protohistorique, et puis ailleurs...}

Sans s'écarter d'emblée de notre univers habituel de référence, et pour débuter par un domaine stable et peu contesté - celui de la poterie -, on notera que la figure exactement cruciforme est présente (fig. 4) :

- dans les registres décoratifs du Rubané haut-rhinois et du Rubané récent du Bassin parisien, placée en motif secondaire : les Graviers 6 à Passy dans l'Yonne (Carré, 1996) ; les Réaudins à Balloy en Seine-et-Marne (Mordant, 1991) ;

- écho probable de motifs similaires inventoriés aussi bien en Hollande (à Sittard, voir Modderman, 1985) qu'en Allemagne où la série céramique de Plaidt se distingue par plusieurs signes en croix, dont la forme latine (Berhens, 1973) ;

- tandis que le trop singulier motif cruciforme aux extrémités ramifiées devrait à nos yeux pouvoir se comparer au dessin similaire observé dans le Cardial catalan (pour Cova Sarsa, voir Marti Oliver, 1990) où, cette fois, sans frein ni complexe, c'est l'homme que l'on identifie. Il ne s'agit pourtant pas de la croix «latine» présente au Mané Lud, mais de la croix grecque, ou «alésée » selon la terminologie de l'héraldique médiévale, à branches symétriques, équilatérales; une comparaison brève avec les gravures de Renegade Canyon aux USA (fig. 7) renforce pourtant la valeur heuristique de l'analogie, car ici le registre ethnographique régional confirme bien la teneur de l'être représenté (Grant, 1983).

- En fait, dans ce même registre formel, la somme véritable et significative des occurrences néolithiques est établie dans la zone balkano-carpathique où une tradition de marquage du fond des récipients, bien identifiée dans la production de la culture Boian-Giulesti de la plaine Braila, s'attache également aux cultures Turdas,
Vinca B, Gradesnica, Karanovo IV-V et Precucuteni I-II, soit une datation dans la première moitié du Ve millénaire pour la plus grande complexité des combinaisons propres aux étapes Vinca et Turdas (Sirbu, Pandrea, 2003). Étonnamment, et malgré l'identité manifeste avec le signe anthropomorphe classique que révèle le catalogue des cruciformes sur un site comme celui de Turdas-Lunca en Roumanie, aucune interprétation franche dans ce sens n'a été établie jusqu'ici.

- Achevons cette rapide enquête sur l'Europe centrale et orientale en indiquant la présence de ce même motif incisé, peint ou modelé, au sein de la thématique décorative des récipients des cultures Herpaly (Raczky dir., 1987), Cucuteni (Mantu, 1998), Baalberg (Zápótocky, 1991) et Vucedol (Sandars, 1985) au long de deux millénaires d'histoire, sans qu'une interprétation claire, là encore, ne s'établisse dans la littérature (voir Haarmann, 2005 pour un tableau synthétique).

- Encore plus faible en tant qu'image démonstrative, la quadripartition de la structure du décor des écuelles et des coupes-à-socle circulaires chasséennes peut être néanmoins versée au dossier, pour mémoire.

- Mais, plus généralement, le thème cruciforme inscrit sur poterie, sans être abondant, est cependant reproduit dans l'Europe entière, du Portugal au Danemark, et en particulier sur les couvercles circulaires de la culture Trichterbecher (TRB) (Ebbesen, 1975).

- Quant au Néolithique proche-oriental, il donne maints exemples de cette quadripartition des récipients par le décor, et de Samara en Irak à Sesklo en Grèce, on ne manqua pas d'établir des cartes de distribution pour telle ou telle composition décorative interne sur assiette ou écuelle prouvant sa large diffusion par l'Anatolie (Settegast, 1986).

Fig. 4 - Représentation schématique du corps humain, les signes cruciformes : pictogramme nord-américain (d'après Kulundi, 1957) ; Innunnguaq des Inuit, Nuvanut canadien (d'après Hallendy, 2001) ; cerfs-volants Maori (d'après Elsdon 1925) ; piquet anthropomorphe, Alaska (d'après Boas, 1883) ; poupées de sommeil, Guatemala (cliché: S. Cassen, CNRS) ; gravures de bateaux montés à Gobustan, Azerbaïdjan (d'après Djafarzade, 1973), sur la rivière Olekma, Russie (d'après Kotchmar, 1995), et à Bardal, nord Trøndelag, Norvège (d'après Gjessing, 1932) ; anthropomorphes à Pera di Crus, Valchiusella, Italie (d'après Seglie et al., 1991), Peñas de Cabrera, Casabermeja, Espagne (d'après Cantalejo et al., 2006), et Molino Manzanez, Espagne (http://rupestre.net; photo: H. Collado Giraldo) ; motifs gravés sur fonds de récipients céramiques à Turdas-Lunca, Roumanie (d'après Sirbu, Pandrea, 2003); antropomorphes sur céramiques à Sounjentck, Caucase (d'après Korenebskii, 2001), et Imiris Gora, Transcaucasie (d'après Masson et al., 1982) ; signe en croix surmontant une gerbe de traits, salle Brunel, grotte Chauvet à Vallon-Pont-d'Arc, Ardèche (d'après Feruglio, Baffier, 2005) ; motifs cruciformes sur céramiques du Néolithique ancien, str. 48 à Maisy, Calvados (d'après Lebolloch et al., 1986), les Graviers 6 à Passy, Yonne (d'après Carré, 1996), LinearBandKeramik (LBK) à Plaidt, Allemagne (d’après Müller-Karpe, 1968), Cova Sarsa, Espagne (d'après Marti Oliver, 1990), et sur céramiques chalcolithiques à Vrbice, République tchèque (d’après Zápótocky, 1991), et Ctidrucice, Slovaquie (Moravské zemské muzeum, Brno) ; corps momifié de Ötzi, Alpes italiennes (d'après Moser et al., 1999) ; galet peint azilien (d’après König, 1987) ; modèle d'habitation Vucedol de Sarvas, Croatie (d'après Sandars, 1985) ; Ballyshannon, Irlande; monnaie gauloise des Volques Tectosages (http://www.loisirs-detections.com/images/28.jpg). 


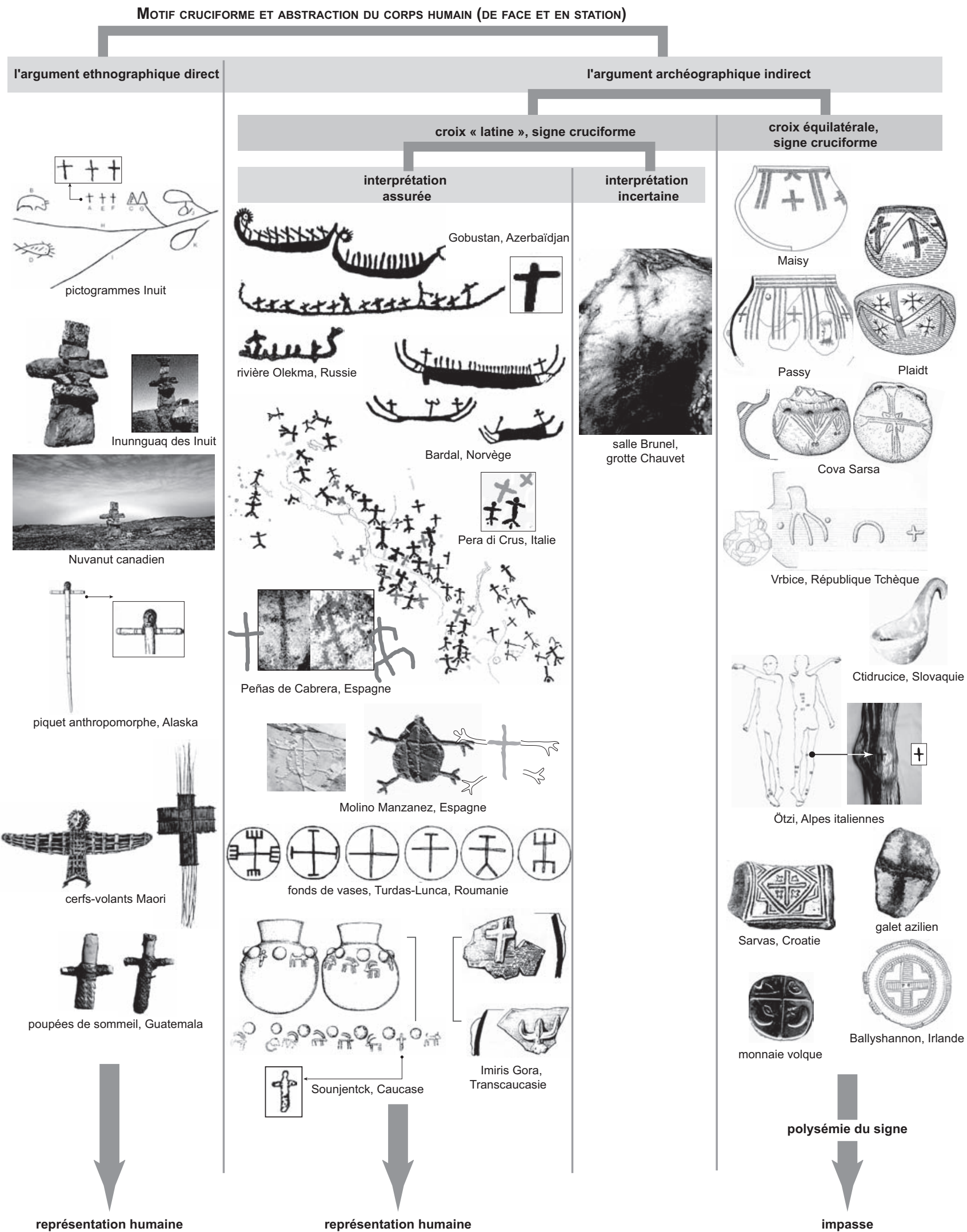


Bien entendu, dès qu'il s'agit de porter un « décor » sur la face externe du fond plat de certains récipients, et donc de le cacher a priori au regard quotidien, dès qu'il s'agit d'organiser un «remplissage » de ces surfaces restreintes, avec régularité la partition du fond sera tributaire, parmi d'autres combinaisons, d'une orientation croisée des tracés continus ou discontinus, ce qui, par ces arrangements géométriques réputés universels dans leur invention, nous éloigne somme toute du but recherché, exception faite, nous l'avons dit, de certains sites néolithiques des Carpathes.

- Plus attractive malgré son égal éloignement de notre zone de compréhension, la thématique «décorative » sur les groupes céramiques de Shulaveri et Arukhlo en Transcaucasie (milieu du $\mathrm{VI}^{\mathrm{e}}$ millénaire av. J.-C.), comportant des décors plastiques anthropomorphes - dont de claires correspondances avec l'Anatolie orientale ont été notées par plusieurs auteurs (Masson et al., 1982, voir le site de Imiris Gora, horizon IV, fig. 39 ; Chataignier, 1995) -, permet cette fois de recueillir d'excellents témoignages du passage alternatif d'une forme humaine aux bras écartés et levés, immédiatement reconnue, à la représentation réalisée par le truchement du seul motif cruciforme.

- La culture nord-caucasienne de Maïkop (à partir de la seconde moitié du IV millénaire) prolonge d'ailleurs ces représentations anthropomorphes, également traitées en relief sur le mode cruciforme "latin » (fig. 4), personnages entourés d'animaux cornus (ovins du type bouc sauvage de montagne) et peut-être de chiens (Korenebsky, 2001). Dans ces deux contextes, l'argument archéographique indirect est recevable, valide.

- Inattendu mais révélateur de la vaste distribution du motif crucifère/cruciforme, que ne documenterait pas d'ailleurs aussi bien le registre décoratif des productions contemporaines de la poterie ouest-européenne dont il nous faut maintenant prendre congé, Ötzi, l'homme ressurgi des glaces alpines après plus de 5000 ans d'enfouissement, présentait également de petits segments croisés, tatoués sur la peau (percée et colorée dans la blessure), et curieusement aux points d'articulation de ses membres : une croix à la cheville gauche au niveau d'une sévère arthrose, une autre à l'intérieur du genou droit en rapport éventuel avec une autre affection dont il souffrait, la branche principale des deux motifs étant orientée selon l'axe longitudinal du corps (Dorfer et al., 1998 ; Moser et al., 1999) (fig. 4).

En passant maintenant à l'ensemble des représentations cruciformes gravées ou peintes sur parois, affleurements ou dalles des tombes en Europe occidentale, le champ des com- paraisons apparaît non seulement plus élargi, mais aussi le plus légitime; s'il s'agit cette fois de la forme "latine » recherchée, elle n'est pas pour autant la mieux datée :

- terrain naturel des comparaisons avec la Bretagne, la Galice comprend de nombreux affleurements où les cruciformes, «latins» ou non, semblent postérieurs au «style atlantique ", leur chronologie s'étendant du Bronze ancien aux premiers âges du Fer (Santos-Estévez, Seoane-Veiga, 2005, p. 42) ;

- sur les mêmes terrains métamorphiques du Portugal, les représentations anthropomorphes protohistoriques seraient ainsi christianisées par adjonction de cruciformes médiévaux sur les mêmes affleurements (Chã da Rapada, voir Martins, 2006, p. 63), la section seule des tracés les différenciant dans le temps (en U ou en V), l'incision supplantant la percussion ;

- plus au sud, quelques trop rares signes gravés dans une tombe mégalithique datée du IVe millénaire à Vilaboa (Shee-Twohig, 1981) ne peuvent pas non plus assurer une correspondance significative durant ces dernières années ;

- ils sont désormais mieux concurrencés dans cette partie de la péninsule Ibérique par un ensemble de gisements peu ambigus, récemment découvert sur le cours de la rivière Guadiana séparant les deux pays (Molino Manzanez en Espagne, fig. 4, voir Cerrato Leyton, Novillo Gonzalez, 2000 et Collado Giraldo, 2004), réputés contemporains du monument portugais précédent pour certains de leurs motifs anthropomorphes;

- mais c'est en Andalousie où le corpus est le plus disert, grâce à des abris comme celui de Peñas de Cabrera (Casabermeja) où un ensemble de peintures ocre-rouge révèle une belle diversité de silhouettes anthropomorphes dont un cruciforme parfait, supposé dater du Néolithique (Cantalejo et al., 2006, p. 108).

- À l'autre extrémité de la péninsule, la question de la datation est encore plus difficile à traiter dans le cas des nombreux «anthropomorphes » de la région catalane où affleurements, dalles de mégalithes et parois des abris ou grottes échangent et répètent le motif, souvent confondu avec la croix chrétienne véritable, mais parfois détaché sans ambiguité de ce dernier symbole religieux (Petra Scripta de Fontcoberta, Peyra Escrita, Cauna de Perellos, etc., voir Abelanet, 1990). Quelle que soit ici la datation, qui peut en effet se révéler assez tardive pour certaines gravures (une date historique est parfois généralisée radicalement à l'ensemble du corpus catalan, voir Galant, 2005), le fait essentiel devant retenir l'attention est cette contiguité régionale formelle entre l'anthropomorphe « réaliste » et le 
signe cruciforme, qui autorise l'œil et la pensée de passer de l'un à l'autre sans aucune difficulté.

- Ce même passage de la forme humaine, reconnaissable par ses unités graphiques constitutives (tête, mains, membres, sexe, etc.), à la figure cruciforme radicale, est bien entendu attesté dans les vallées alpines de l'Italie du Nord où les exemples de gravures sur affleurements et dalles erratiques ne manquent pas, illustrant la juxtaposition des deux formules sur un même support, ou en un même emplacement (fig. 4), comme à Pera dij Crus (Valchiusella), San Giono (Valle Susa) (Seglie et al., 1991), à l'image de ce que nous venons de voir au Portugal (Chã da Rapada).

- L'homme aux bras étendus à l'horizontale (fin du IV $^{\mathrm{e}}$-début du III $^{\mathrm{e}}$ millénaire) peut d'ailleurs attirer et fixer les représentations postérieures, et la croix médiévale chrétienne se poser ostensiblement aux côtés de l'anthropomorphe comme à Foppe di Nadro (Sansoni, Marretta, 2002).

- Un tel processus de simplification et d'abstraction est en tout cas fort bien illustré à travers les figurines pendeloques chypriotes appartenant à la culture Erimi (IV millénaire av. J.-C.), où les petits personnages sculptés (fig. 6), féminins ou masculins, jambes jointes et bras étirés sur les côtés, mains et visages dessinés, passent insensiblement vers la forme brute "en croix » (sans qu'il soit nécessaire ici de concevoir un processus évolutif à valeur chronologique) où tout caractère anatomique, sexuel, a disparu (Karageorghis, 1976 ; Crewe et al., 2002).

C'est, en somme, ce qu'il était encore possible d'observer chez les indigènes des Hautes Terres du Guatemala (autour du lac Atitlan), qui bricolaient de petites poupées femelles (dites «poupées Maya»), habillées, de $2 \mathrm{~cm}$ ou $3 \mathrm{~cm}$ de long, les bras en croix, les jambes jointes (fig. 4), poupées disposées sous la tête durant le sommeil et qui permettaient au dormeur de se débarrasser de toutes ses angoisses; un simple agencement cruciforme de minuscules brindilles liées par des fils colorés faisait aussi l'affaire ${ }^{7}$;

- meilleurs, car plus démonstratifs pour notre sujet et notre projet, sont les personnages embarqués sur les très

7. La mise en «mondialisation" de l'artisanat traditionnel de ces régions permet aujourd'hui d'acquérir ces mêmes poupées via Internet ou dans les magasins «ethniques» des plus riches pays industriels. Mais la forme en croix originelle - sans que l'Église chrétienne ne soit intervenue dans ce dessin - est de plus en plus concurrencée par la sihouette aux jambes disjointes, entraînant par voie de conséquence une sexualisation prononcée (les jambes écartées seront celles des hommes-poupées). anciens navires gravés en bordure de la mer Caspienne par des chasseurs-cueilleurs de Gobustan en Azerbaïdjan (Djafarzade, 1973 ; Anati et al., 2001) ;

- et encore, au long des grands fleuves sibériens et de leurs affluents (voir le tableau comparatif des embarcations montées établi par Kotchmar, 1995, p. 45), ces silhouettes résolument anthropomorphes, ou bien se réduisant à de simples traits verticaux - sur un mode que nous pourrions reconnaître aisément en différentes régions du monde (voir infra, p. 225 et fig. 4 et 9) -, ou bien produisant ces dessins en croix aux modules divers, que nous savons cette fois beaucoup plus rares mais si expressifs pour dire les humains ;

- bien entendu, les représentations de bateaux de l'âge du Bronze scandinave réunissent d'autres occurrences aussi convaincantes pour signifier l'homme (Fet, Fet, 1941), et à côté des habituelles barres verticales simples qui signalent les embarqués, on trouve des anthropomorphes armés et casqués, d'autres sautant (les «voltigeurs"), d'autres encore levant les bras au ciel (les « adorants») se plaçant à côté des figurations en croix.

\section{LE SIGNE CHRÉTIEN}

Cette première étape franchie et ces données archéologiques accumulées pour constituer un premier corpus de comparaisons, reprenons du recul et voyons ce qu'une brève historiographie du signe, en des morceaux choisis, peut nous apprendre sur cette décision de rejeter ou de s'approprier l'interprétation anthropomorphe par référence implicite ou explicite avec le symbole chrétien, une alternative fort ancienne qui affecte encore aujourd'hui l'interprétation des gravures préhistoriques étudiées dans la moitié ouest de la France.

Le préhistorien G. de Mortillet a ainsi tenté le premier de restituer le parcours du signe de la croix avant le christianisme (Mortillet, 1866) ${ }^{8}$. Mais son bref tour d'horizon ne suffit plus aujourd'hui à alimenter notre débat. Choisissant, là des motifs croisés incisés sur le fond de vases étrusques, ici un motif cruciforme parmi de nombreux signes géométriques au sein des tombes de Villanova (Italie), ou encore une monnaie gauloise portant une croix aux extrémités

8. «Cette idée s'est tellement généralisée, tellement imposée, qu'on a admis comme un axiome en archéologie que la Croix est un excellent critérium pour reconnaître ce qui est postérieur au Christ, ce qui appartient à l'ère actuelle. Pourtant ce critérium n'a aucune valeur. La Croix, la vraie Croix, se trouve sur de nombreux objets bien antérieurs à la venue de Jésus-Christ... " (Mortillet, 1866, p. 1). 
arrondies (probable rouelle avec croix enfermée dans un cercle, transformée en croix isolée), son inventaire trop restreint dans le temps et seulement illustré de "croix » équilatérales ${ }^{9}$, ainsi que des choix mal posés pour fonder la cohérence interne de son article, ne font qu'imager une opinion mais ne permettent pas de former la structure close et temporairement inattaquable d'une "démonstration" qui autoriserait la contestation du sentiment dominant à l'époque, et toujours vivace à l'heure où nous écrivons ces lignes, quant à la datation tardive du motif mis en cause.

À l'inverse, de nombreux missionnaires officiant au cours des deux siècles passés ont tenté d'expliquer par des influences chrétiennes les nombreuses représentations de croix repérées parmi les pictogrammes d'Amérique du Nord (Vazeilles, 1995) :

- pour les Sioux, les croix «grecques » représentent les Quatre Vents, avatars du Grand Esprit ;

- pour d'autres tribus, les quatre points de l'horizon seront désignés par une croix latine, représentant une libellule, l'un des messagers de l'Oiseau-Tonnerre ;

- pour les Ojibwas, une croix « latine » renversée représente les actions du héros civilisateur Manabozo tandis que des séries de petites croix désigneront les habitations ojibwas, les wigwams ;

- pour les Shoshoneans elles représenteraient des étoiles ;

- enfin, signe tribal des Cheyennes ces croix dessinées désignent le nombre de prisonniers, d'exploits guerriers, etc. dans les winter-coups sioux.

On s'accorde à dire que seul le contexte, au sens le plus large (oral, social, technique, etc.) peut éventuellement différencier ces nombreuses croix ; mais, dans bien des cas, une analyse graphique un tant soit peu poussée permet toutefois de les différencier, sans l'aide de la parole: une croix inuit pour signifier l'oiseau n'a rien d'un anthropomorphe à qui sait regarder la scène, la composition graphique ou la forme du signe, et la polysémie dudit signe qu'il est habituel d'avancer pour couper court à toute analyse synthétique sur le sujet ne vaut que le temps de contourner l'écran parfois involontairement construit par nos collègues ethnologues, quand ils s'éloignent de

9. Parmi les différentes croix retenues par les historiens, rappelons : la crux commissa ou crux summissa qui était en forme de $\mathrm{T}$; la crux capitata ou immissa qui avait une barre traversant le poteau à son sommet; la crux decussata qui avait deux montants égaux et obliques comme un X, appelé aujourd'hui "croix de Saint-André " par allusion au martyre que subit ce chrétien. ces questions radicales de figuration, nous cachant par omission la simplicité des images pourtant distinguées par ces populations.

Une illustration tirée des sociétés du Nord canadien (Nunavut) pourra d'ailleurs servir d'argument ethnographique direct (fig. 4). En ces terrains au climat si hostile, les Inuit et leurs ascendants ont édifié, au cours des trois ou quatre derniers millénaires, une belle variété de structures en dalles, comme autant de messages adressés au voyageur de passage. Ces inuksuit indiquent un point, dirigent les caribous vers un lieu de chasse, soulignent un concours entre groupes voisins, affichent un élément spirituel ou un bien surnaturel. Un inuksuk (au singulier) est un « homme par procuration ${ }^{10}$, dans tous les sens du mot, apportant un réconfort au voyageur solitaire, des informations vitales en cas de désorientation, une cache pour la viande, un endroit où l'on peut se reposer... et un lieu de recueillement spirituel pour les croyants. Les terrains et les pierres disponibles influencent la construction, la facilitant ou la compliquant. L'inuksuk est généralement constitué de pierres plates et minces disposées les unes par-dessus les autres, mais il peut également être aménagé avec une seule dalle dressée ou être formé d'un petit monticule de pierres rondes. Il arrive fréquemment que ces «sculptures» ne soient pas exclusivement formées de pierres et que des ossements de cétacés ou des fragments de bois flottés se retrouvent mêlés à la composition finale. Les plus hauts dépassent $2 \mathrm{~m}$. Et parmi ces structures distinguées, l'innunnguaq, qui a une forme ressemblant à celle de l'humain, mais qui «n'agit pas à la place de l'humain » (Hallendy, 2001), indique la présence d'un campement inuit. Ici la forme voulue est celle de l'homme, un humain cruciforme (fig. 4).

Mais revenons aux représentations du Mané Lud et à la discussion ouverte en Europe par G. de Mortillet: si ce motif cruciforme pose un problème archéologique local, mais aussi total, en raison d'un objet-signe au statut mal résolu, à la situation originelle confuse, et par le fait qu'il demeure à l'heure d'Internet le centre de conflits idéologiques au sein de la chrétienté occidentale après avoir été déjà contesté dès la naissance du christianisme, exigence nous est faite de résumer quelques forces en présence :

- la croix «historique» est à la fois le crucifié en personne et son image, sa «relique » et sa « représentation », le corps divin et son image, écrivait L. Marin (1993,

10. Le terme inuksuk est un dérivé de Inuktitut (en langue inuit), morphèmes Inuk («être humain») et -suk («agir comme »-suit) (Hallendy, 1994). 
p. 226). Aussi ce «signe », où tendent à s'identifier signifiant et signifié, implique une double adoration, comme au Christ lui-même et à sa parfaite image. Cette fusion en présence, augmentative d'une puissance, confine aussitôt à une confusion :

- on peint en effet des croix dans les églises médiévales (au plan de croix «latine») pour trois raisons selon J. de Voragine (1255) : 1) pour la terreur des démons ${ }^{11}$; 2) comme marque de triomphe, car les croix sont les étendards de Jésus-Christ et les insignes de son triomphe ; 3) pour représenter les apôtres ;

- au-delà de cette multiplication des charges signifiantes, la croix est ce premier emblème commun sous lequel s'est regroupé tout l'Occident chrétien lorsque fut prêchée la première croisade; puis, dès le milieu du XIV ${ }^{\mathrm{e}}$ s., les combattants français et anglais se reconnaissent grâce à des croix de couleurs différentes, un exemple bientôt imité par d'autres nations (fig. 5) ;

- et après tant de siècles écoulés, et alors que le temps des sociétés est aujourd'hui accéléré comme jamais il ne le fut dans le passé, la croix demeure dans son usage ou dans sa réfutation un perpétuel objet d'identité, de litige, de revendication, de contestation ou de confiscation du symbole, dont l'illustration la plus étonnante - au-delà du cas ponctuel et superficiel du très médiatique Da Vinci Code - réside dans la polémique opposant l'Église catholique à la puissante Watch Tower Bible and Tract Society of Pennsylvania, autrement dit les Témoins de Jéhovah ${ }^{12}$.

Parce que cet article, bien malgré lui, risque de donner des arguments à des adhérents toujours en quête d'appuis scientifico-archéologiques pour étayer leur thèse, et parce que le problème posé nous intéresse aussi au premier chef dans le cadre de ce processus de reconnaissance, obligation nous est faite de développer quelques attendus pour éclairer le lecteur.

11. «[...] c'est-à-dire afin que les démons qui en ont été expulsés soient effrayés à la vue du signe de la croix et n'aient plus la présomption d'y rentrer; $[\ldots]$ donc c'est pour montrer que ce lieu est sous la domination du Seigneur qu'on y peint des croix [...]. C'est une figure de ce passage de la Genèse (XXVIII) que Jacob érigea la pierre, qu'il avait mise sous sa tête, comme un monument, c'est-à-dire, comme un monument public, digne de mémoire, et triomphal [...] ; car ces douze lumières placées devant les croix signifient les douze apôtres qui, par la foi du crucifié, ont éclairé l'univers " (Voragine, 1255, p. 454).

12. On pourra se référer au site officiel américain (http://www.watchtower.org/library/rq/article_11.htm), dans lequel la société Watch Tower fustige les adorateurs de la croix; en réalité, une nébuleuse de sites-satellites rédigés dans de nombreuses langues, sous d'autres nominations et sous d'autres prétextes, développe le dogme de l'irréalité de la croix.

\section{RÉFUTATION DE LA CROIX}

Jusqu'à la fin des années 1930, la Société Watch Tower représentait Jésus mourant sur une croix traditionnelle, mais finit par éliminer tant la croix que le nom de Jésus sur la page de couverture de ses opuscules de propagande et de liaison, considérant finalement que les Églises sont «impures» du fait qu'elles emploient la croix comme symbole de la mort du Christ. Jouant sur les mots, s'aidant de l'archéologie européenne (et G. de Mortillet sera malgré lui de la partie...) ou eurasiatique, et convoquant des témoignages biaisés mais vraisemblables, arguant de la représentation originelle du poisson ( $\mathrm{II}^{\mathrm{e}} \mathrm{s}$.) et du bon berger $\left(\mathrm{III}^{\mathrm{e}} \mathrm{s}\right.$.) comme symboles du Christ, l'objet «croix » est réfuté au motif principal que le texte (grec) du Nouveau Testament nous parle de stauros, simple poteau vertical planté dans le sol, et que le signe en croix serait, quoi qu'il en soit, bien antérieur au christianisme (et ici les exemples ne manquent pas à l'argumentaire, depuis l'Inde et sa svastika à l'Égypte et sa «croix ansée » dite Ankh, des Celtes à la croix cerclée aux Chaldéens munis de leur fameux Tau mystique issu du dieu Tammuz) et qu'il est en conséquence un symbole païen à combattre.

Effectivement, le mot grec stauros désigne fondamentalement un pieu ou un poteau, mais ce qui n'est pas dit à travers ce premier argument, c'est que le mot désigne également et souvent une construction plus complexe, comme une croix. Le mot latin crux, habituellement rendu par " croix ", était également employé pour désigner un simple poteau, mais les Romains exécutaient bel et bien des prisonniers sur des croix, et l'on appelait patibulum la barre transversale que les esclaves qui devaient être exécutés portaient habituellement jusqu'au lieu d'exécution (ici, l'Église a beau jeu - dans son Encyclopédie catholique - de renvoyer vers les auteurs classiques : Sénèque, De Vita Beata, 19:3 et Epistola, 101: 12 ; Tacite, Historiae, IV, 3).

La croix en tant que symbole païen adopté plus tard par les Églises (deuxième argument de la secte des Témoins de Jéhovah) est moins bien réfutée par Rome, les théologiens supposant a priori que la croix n'évoquait pas pour les chrétiens du I ${ }^{\mathrm{er}} \mathrm{s}$. quelque signification païenne antérieure, mais qu'elle a bel et bien fini par représenter le Christ et son message, tant pour les croyants que pour les non-croyants. Face à l'impressionnante liste archéologique avancée par la secte, qui se vante d'attendre sans anxiété les futures fouilles qui plaideront en sa faveur, l'Église reste indisposée, ne relevant aucunement - tout au moins dans le «dialogue » à distance diffusé sur Internet ainsi que par fascicules 


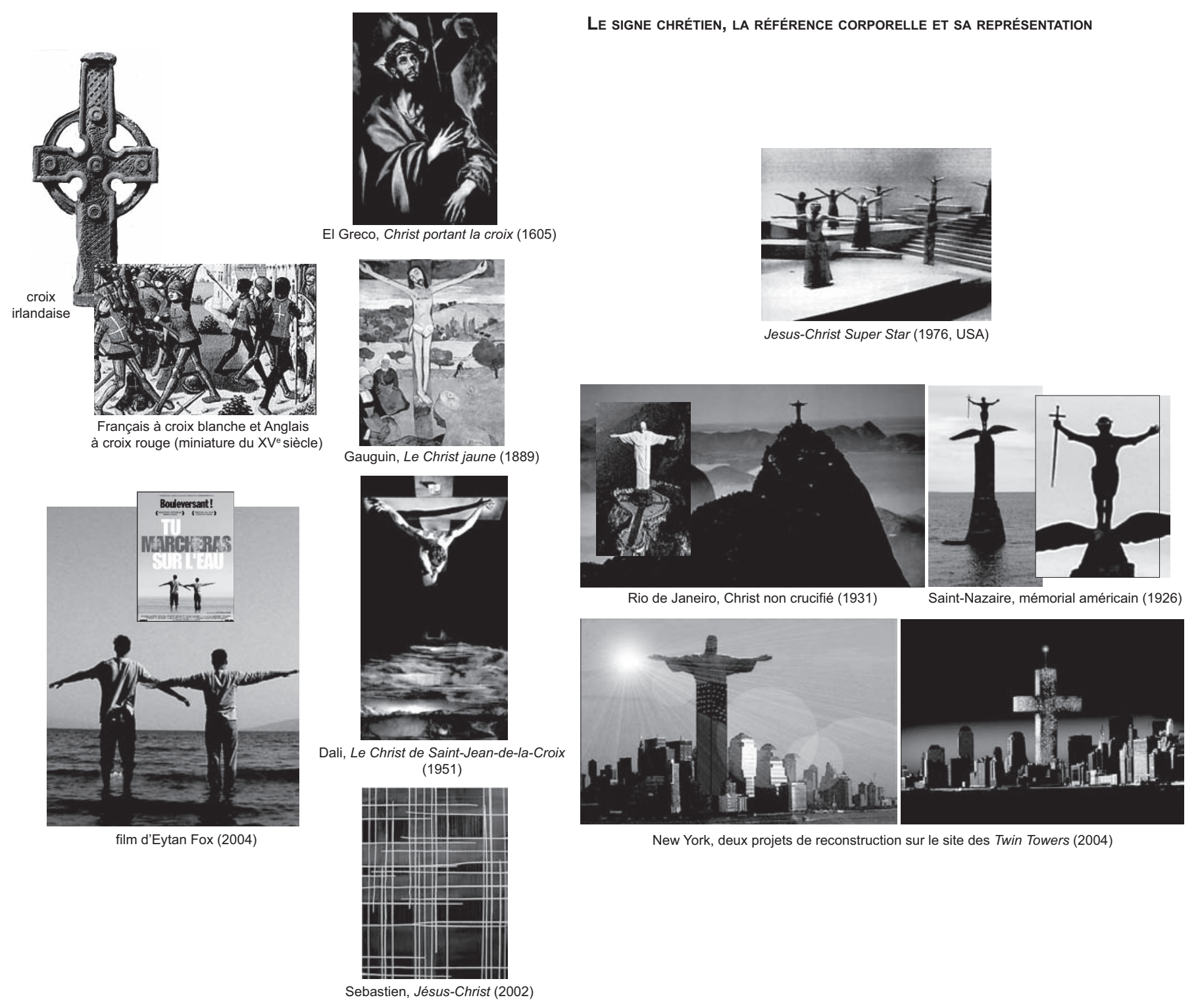

Fig. 5 - La croix chrétienne, une référence au corps immobile, représentation toujours actuelle : croix irlandaise du $X^{e}$ s. (cliché : S. Cassen, CNRS) ; combat entre Français à croix blanche et Anglais à croix rouge (miniature du XVe s., BNF) (http://perso.wanadoo.fr/huchehault) 15 vetarouez.htm) ; affiche du film Walk on water de Eytan Fox, Israël, 2004 ; tableaux du Christ en croix : El Greco, Christ portant la croix, 1605 ; Gauguin, Le Christ jaune, 1889 ; Dali, Le Christ de Saint-Jean-de-la-Croix, 1951 ; Sebastien, Jesus-Christ, 2002 (clichés : S. Cassen, CNRS) ; extrait du film Jesus-Christ Super Star dirigé par Norman Jewison (http://fr.wikipedia.org/wiki/Norman_ Jewison) ; le Christ dominant Rio de Janeiro, Brésil (cliché : S. Cassen, CNRS) ; statue de soldat à l'épée cruciforme commémorant le débarquement des premières troupes américaines à Saint-Nazaire en 1917 (cliché : S. Cassen, CNRS) ; projet de bâtiment en croix ou en forme de Christ non crucifié pour remplacer les Twin Towers à New York (http://www.wtcsitememorial.org/).

interposés - le processus de confusion opéré entre le motif cruciforme équilatéral (la croix grecque, la svastika, etc.) et le motif asymétrique (la croix « latine»).

Pour finir, passons rapidement sur le poteau de supplice (la croix) qui devait être un sujet de honte (troisième argument), et pour cela rejeté en tant que symbole commémoratif, car il est vrai que les Juifs considéraient l'exécution sur la croix comme une façon de mourir ignominieuse, une malédiction, tout espoir de résurrection étant retiré au supplicié. De là cette impossibilité psychologique pour 
les adeptes de Jéhovah - mais aussi pour l'Église chrétienne des quatre ou cinq premiers siècles - à considérer la croix comme une représentation autrement que détestable, puisque glorifiant l'acte inique qu'elle représentait.

Retenons l'essentiel: l'usage du supplice de la crux n'apparaît pas à Rome avant les guerres puniques (III ${ }^{\mathrm{e}}$ et $\mathrm{II}^{\mathrm{e}}$ s. av. J.-C.), et Tertullien ( $\mathrm{II}^{\mathrm{e}}$ s. apr. J.-C.) le fait remonter à l'histoire de Regulus (Ernoult, Meillet, 1967). En latin, le mot crux désignait un gibet ou une potence. Cela pouvait être un simple pieu, voire le timon d'un char auquel on attachait la victime les mains liées derrière le bois. C'était le crux simplex.

À la fin du I $^{\text {er }}$ s. av. J.-C., la crucifixion est adoptée comme punition officielle pour les non-Romains, punition limitée à certaines transgressions. Au départ, ce n'était pas une méthode d'exécution, mais seulement une punition. De plus, seuls les esclaves convaincus de certains crimes étaient punis de crucifixion. Durant cette première période, une poutre de bois, connue, nous l'avons dit, comme la furca ou le patibulum, était placée sur la nuque de l'esclave et liée à ses bras (Tzaferis, 1985). Seulement, en ce qui concerne la crucifixion de Jésus, les sources profanes ne semblent pas permettre de trancher quant à la forme précise de la croix, crux immissa $(\dagger)$ ou crux commissa $(\mathrm{T})$. Enfin, les historiens de l'Antiquité ne disent pas non plus pourquoi les Romains inventèrent ou adoptèrent cette forme en bois.

En cela, la croix « latine» porte bien son nom, et si elle demeure fort discrète dans les contextes paléochrétiens ${ }^{13}$, son culte est très vite lié à celui de la famille impériale (Le Goff, 1985 ; Lucena Martin, 1980) ; son « origine » occidentale est vraisemblable, en s'opposant à la croix « grecque » qui, en effet, limitée au monde orthodoxe après le schisme des Églises, est cependant d'une signification historique bien plus élargie et d'une date plus ancienne dans le seul Caucase (Charachidzé, 1994, p. 221) ou dans le Proche-Orient, et en réalité d'une répartition quasi planétaire quand on la réduit au motif cruciforme/crucifère des décors incisés, tatoués, peints, tissés, etc. ; moyen prolifique par lequel il devient alors chose aisée de comparer entre elles les seules images pour argumenter dans le sens désiré.

En résumé, la tradition chrétienne a prodigieusement enrichi le symbolisme de la croix, en condensant dans

13. En 312, l'empereur Constantin, à la veille d'engager une bataille décisive et victorieuse contre Maxence, sous les murs de Rome, vit en plein jour une croix dans le ciel ; la nuit, il vit cette fois en songe le Christ lui-même l'incitant à faire représenter la croix sur une enseigne ; l'année suivante, par l'édit de Milan, Constantin ouvrit la voie à la reconnaissance des fidèles de ce signe et à sa propre conversion (Le Goff, 1985, p. 301). cette image l'histoire du Salut et de la Passion. Si la croix symbolise le crucifié, le Christ, le Sauveur, le Verbe, la deuxième personne de la Trinité, elle est pourtant plus qu'une figure de Jésus-Christ, elle s'identifie à son histoire humaine, voire à sa personne (Chevallier, Gheerbrant dir., 1969).

La hiérarchie catholique n'eut de cesse de faire célébrer des fêtes de la Croix, créant l'Invention de la Croix, répétant l'Exaltation de la Croix, et, dès l'origine, chaque ressemblance, fût-elle fortuite, était bonne à prendre pour asseoir la prééminence du signe: ainsi Justin dans son Apologie $(1,55)$ passe en revue tout ce qui porte l'image de la croix ${ }^{14}$ : l'énumération des cruces dissimulatae comporte la charrue, l'ancre, le trident, le mât du navire avec antenne, la croix gammée, etc., en une liste qui ne semble guère éloignée du raisonnement par analogie quand il est dans notre discipline une surestimation d'une forme isolée.

On voit par de telles accumulations combien il devenait difficile de démêler un écheveau déjà compliqué à son origine, un signe sur lequel un des plus grands poètes, Dante, pouvait rester sans voix ${ }^{15}$. Un signe qui s'inscrit pourtant dans une herméneutique que l'archéologie peut aborder.

\section{L'HOMME-CROIX}

Vue d'un peu loin, l'image de la croix se confond donc avec la silhouette de l'humain. Cette relation homothétique semble si naturelle, allant tellement de soi entre le sujet et son ombre portée, qu'on recherche peu la raison du transfert, ses écarts par rapport au modèle, et finalement la rareté du signe "latin » dans les représentations anthropomorphes de par le monde. Sans vouloir prétendre à la résolution de l'énigme, cet essai d'archéologie d'une image permettra de poser les bases d'une réflexion plus générale dépassant le propre signe néolithique en question :

14. «[...] veuillez réfléchir un instant, et voyez si dans ce monde on ne peut rien faire sans ce signe, si sans lui le moindre commerce est possible entre les hommes? Peut-on fendre les ondes sans que, formé de la vergue et du mât, il brille comme un trophée ? Peut-on tracer un sillon sans la croix de la charrue? Tous vos pionniers, comme au reste tous les artisans et tous les manœuvres, ne peuvent travailler sans des instruments qui affectent sa forme. L'extérieur même de l'homme ne diffère de celui des animaux que parce que son corps se tient droit et qu'il peut étendre les mains en croix. Et ce nez, proéminent organe de la respiration vitale, ne trace-t-il pas encore une croix au milieu du visage? " (Apologie, 1, 55).

15. La Divine Comédie, 1321 : "Sur cette croix le Christ resplendissait tant que je ne sais trouver d'image pour le représenter [...]». 
- tout d'abord en valorisant l'espace du commencement ;

- en orientant, ensuite, cet espace par rapport à mon corps, ce corps en rapport avec le monde ;

- en disposant, enfin, ce corps suivant la station ou son mouvement, suivant une opposition fondamentale que devra traduire la dynamique des tracés et qui déterminera nos propositions à venir quant au sens plausible des associations de signaux.

Entendons-nous sur deux préalables :

- d'une part, l'espace n'est pas le lieu (réel ou logique) dans lequel les choses se disposent, mais le moyen par lequel la position des choses devient possible ;

- d'autre part, l'expérience de l'espace est entrelacée avec tous les autres modes d'expérience et toutes les autres données psychiques, et nous ne saurions les oublier en les posant à l'écart (Merleau-Ponty, 1945).

De là, cette première interrogation dictée par ce chercheur, ce premier accord qu'il nous faut passer avec le lecteur: l'espace du rêve, l'espace mythique, sont-ils de véritables espaces, peuvent-ils être et être pensés par euxmêmes, ou bien ne présupposent-ils pas comme condition de leur possibilité l'espace géométrique ("archéométrique », ajouterions-nous) et avec lui la pure conscience constituante qui le déploie?

En sorte que s'il fallait s'accorder à la critique négative adressée à de tels essais d'interprétation, au pessimisme quant à la pertinence scientifique de l'histoire reconstruite à partir de ces gravures, de même que pour destituer l'expérience mythique, celle du rêve ou celle de la perception de toute valeur positive, pour réintégrer les espaces à l'espace géométrique convenu, ne faudrait-il pas en somme nier que l'on ne rêve jamais, que l'on ne soit jamais fou, ou que l'on ne perçoive jamais pour de bon ?...

Mais dans cet espace reconnu en tant que tel, il y a un autre sujet au-dessous de moi pour qui un monde existe avant que je sois là et qui y marquait ma place. Cet esprit captif ou naturel, rapporte M. Merleau-Ponty, c'est mon corps; non pas le corps momentané qui est l'instrument de mes choix personnels et se fixe sur tel ou tel monde, mais le système de "fonctions » anonymes qui enveloppent toute fixation particulière dans un projet général. L'espace, la perception, marquent au cœur du sujet le fait de sa naissance, l'apport perpétuel de sa corporéité, "une communication avec le monde plus vieille que la pensée... » (id., p. 294).

Et le corps de l'homme et ses membres constituent le système de référence sur lequel sont transposées indirecte- ment toutes les autres différences spatiales. C'est souvent, au surplus, la forme de cette projection qui doit contenir la réponse à la question mythique de l'origine et qui domine par conséquent la cosmographie et la cosmologie mythiques dans leur totalité (Cassirer, 1924, p. 117).

Placez-vous simplement: votre visage est immobile face à l'océan, les bras sont ouverts pour embrasser l'immensité, la ligne du rivage court parallèle à l'alignement joignant une main à l'autre, et derrière vous se tiennent la terre et l'habitation. Tournant sur soi pour s'ouvrir à la courbe du soleil, au prolongement de votre bras gauche celui-là s'élève à l'horizon, et au bout de votre main droite il disparaîtra sous la mer; face à vous sera la chaude lumière de la journée, tandis que votre dos sera refroidi par l'ombre, exposé à la froidure d'un vent d'hiver... Enfin votre corps et l'apesanteur désignent un haut valorisé, à votre tête et bien plus haut que vous ; et un bas dévalorisé par la chute où les pieds touchent terre, pire même, au plus bas que vous.

Voilà des directions simples ordonnées par notre corps déployé, des directions aussitôt orientées, les orients, qui ne sont pas ceux qu'utilisent le processus de repérage.

Et de même que, lieu de la présence sociale, découpée spatialement dans un ensemble social, l'habitation spatialise la société en même temps qu'elle socialise l'espace, le corps spatialise le monde et l'oriente.

Nous reviendrons plus loin, et dans Le Mané Lud en mouvement, à propos du motif quadrangulaire, sur ce passage de l'orientation subjective à l'orientation objective par la fixation d'un centre déterminé à l'aide des directions cardinales. Il nous importe davantage, dans l'immédiat, de fixer la représentation de l'humain debout.

Les éléments anatomiques les plus simples qui définissent graphiquement un corps vu de face ${ }^{16}$, une silhouette humaine, sont :

- le rachis et les membres détachés (bras, jambes), le rachis céphalique étant dégagé ;

- la tête, les mains, les organes génitaux, pourront éventuellement préciser le dessin si un modèle l'exige ;

- les pieds et les cheveux peuvent encore le compléter, mais le travail est accompli, et des vêtements portés et des objets saisis n'ajouteront plus rien à l'économie du tracé.

Laissons les êtres fabuleux et leur taxinomie, qui conjoignent des termes antinomiques ou substituent des éléments

16. La représentation de profil, plus délicate à abstraire pour signifier l'humain, sera volontairement laissée de côté, faute d'être reconnue en Europe atlantique pour le Néolithique. Fessier proéminent et seins seront privilégiés pour signifier la personne durant le Paléolithique ou encore parmi les derniers chasseurs-cueilleurs d'Anatolie. 
appartenant à d'autres créatures, ou exagèrent un détail anatomique; quoi qu'il en soit, ils sont perçus par cette même dynamique de présentation qui prévaut à la reconnaissance d'un anthropomorphe.

Cette étape franchie, une alternative remarquable, car universelle, scinde l'ensemble des représentations connues (fig. 6) :

- le premier terme détermine un corps debout (plus rarement assis) aux jambes confondues, strictement jointes ou écartées (mais alors sans flexion latérale des membres inférieurs) ;

- le second terme, le plus courant, rassemble toutes les figurations des membres inférieurs écartés, fléchis à la hauteur de leurs points articulatoires (bassin, genou, cheville).

Cette distinction, posée sur la base des membres locomoteurs, doit être répétée en réalité à l'échelle des bras qui suivent une partition homologue en fonction de leur position ; on peut donc les trouver :

- soit étirés sur les côtés, à l'horizontale, ou inclinés vers le haut ou bien encore vers le bas ;

- soit pliés aux articulations (épaule, coude, poignet), mains dirigées vers le haut ou vers le bas, symétriquement disposés, ou plus rarement asymétriques (un bras cassé vers le haut, l'autre vers le bas) dans les représentations des sociétés «traditionnelles » actuelles ou du plus lointain passé.

Ce pas accompli, on s'entendra sur un constat supplémentaire, assez banal : c'est l'ensemble des membres qui anime le corps vertical.

Et deux pieds joints ne disent pas le mouvement mais son opposé : la station, la station en tant qu'arrêt dans le mouvement vital qui porte l'homme, une opposition entre la dispersion «acquisitoire » dans l'étendue et le recueillement spatial dans l'habitation, car la station signifiant l'immobilité ne peut se passer du concept de mouvement par rapport auquel elle se définit négativement ${ }^{17}$.

a) L'arrêt, la position statique, le repos, permettent la concentration, la réflexion, la méditation, le recueillement face au temps qui s'écoule ;

- la station autorise le processus d'orientation visuel ;

- elle annule sans effort l'apesanteur en lui résistant par le corps vertical immobile et centré.

b) Le mouvement met un terme à la rêverie, au temps suspendu, à la perception du mouvement des autres ;

17. Pour une application du concept à la Préhistoire récente atlantique, voir Boujot et al., 1995 ; Vaquero Lastres, 1999.
- il joue désormais de l'apesanteur en contrant la chute sans cesse répétée de l'homme marchant, courant ou dansant ;

- le corps est en branle, décentré, la pensée déconcentrée, distraite par le mouvement; et l'humain dansant se désoriente par sa volonté propre ${ }^{18}$.

Une opposition que l'historien retrouve sous d'autres aspects quand l'homme gesticulant devient si suspect aux clercs du Moyen Âge puisqu'il évoque l'acteur du théâtre païen et le possédé du démon qui inscrit ses mouvements dans l'espace, lieu de rencontre entre l'homme biologique et l'homme social (Le Goff, 1985, p. 126).

Bien entendu, tout ceci n'a aucun sens si l'on n'accorde pas au corps, même perçu en état de veille, une valeur emblématique.

Entre nos émotions, nos désirs et nos attitudes corporelles, il n'y a pas seulement une connexion contingente ou même une relation d'analogie, précise M. Merleau-Ponty: si la déception de ne pas être compris à travers ce texte sur l'archéologie des images me fait tomber de haut, ce n'est pas seulement parce qu'elle s'accompagne de gestes de prostration en vertu des lois de la mécanique «nerveuse », ou parce que je découvre entre l'objet de mon désir et mon désir luimême le même rapport qu'entre un objet haut placé et mon geste vers lui ; le mouvement vers le haut comme direction dans l'espace physique et celui du désir vers son but sont symboliques l'un de l'autre, parce qu'ils expriment tous deux la même structure essentielle de notre être comme être situé en rapport avec un milieu, une structure qui donne seule un sens aux directions du haut et du bas dans le monde physique.

L'orientation de l'homme exige, en résumé, un triple accord :

18. Proche de notre méthode et de cette herméneutique des images, le travail remarquable de Ingrid Fuglestvedt sur les gravures scandinaves se fonde néanmoins sur une autre distinction : la mort serait la cause de la taille égale des personnages-bâtons dans le bateau, la mort qui leur fait perdre toute personnalité et oblitère toute mention de la tête, des membres, du sexe ; et c'est en opposition à cet anonymat provoqué par la mort que les guerriers ou les musiciens, ou encore le fameux voltigeur - un personnage qui saute au-dessus de ses compagnons - seraient l'antithèse de la mort, traduisant, par leur comportement, vie, joie, expression corporelle, provoquant justement cette mort frôlée et dont on prend aussitôt, vainqueur, un congé temporaire (Fuglestvedt, 1999, p. 30)... Mais, outre que ces représentations de bateaux ne peuvent être systématiquement postulées comme des bateaux de trépassés, la distinction que nous posons entre représentations humaines statiques et dynamiques ainsi que leur hiérarchie de placement dans l'embarcation pourraient mieux rendre compte des différences entre les scènes pour enfin les interpréter. 

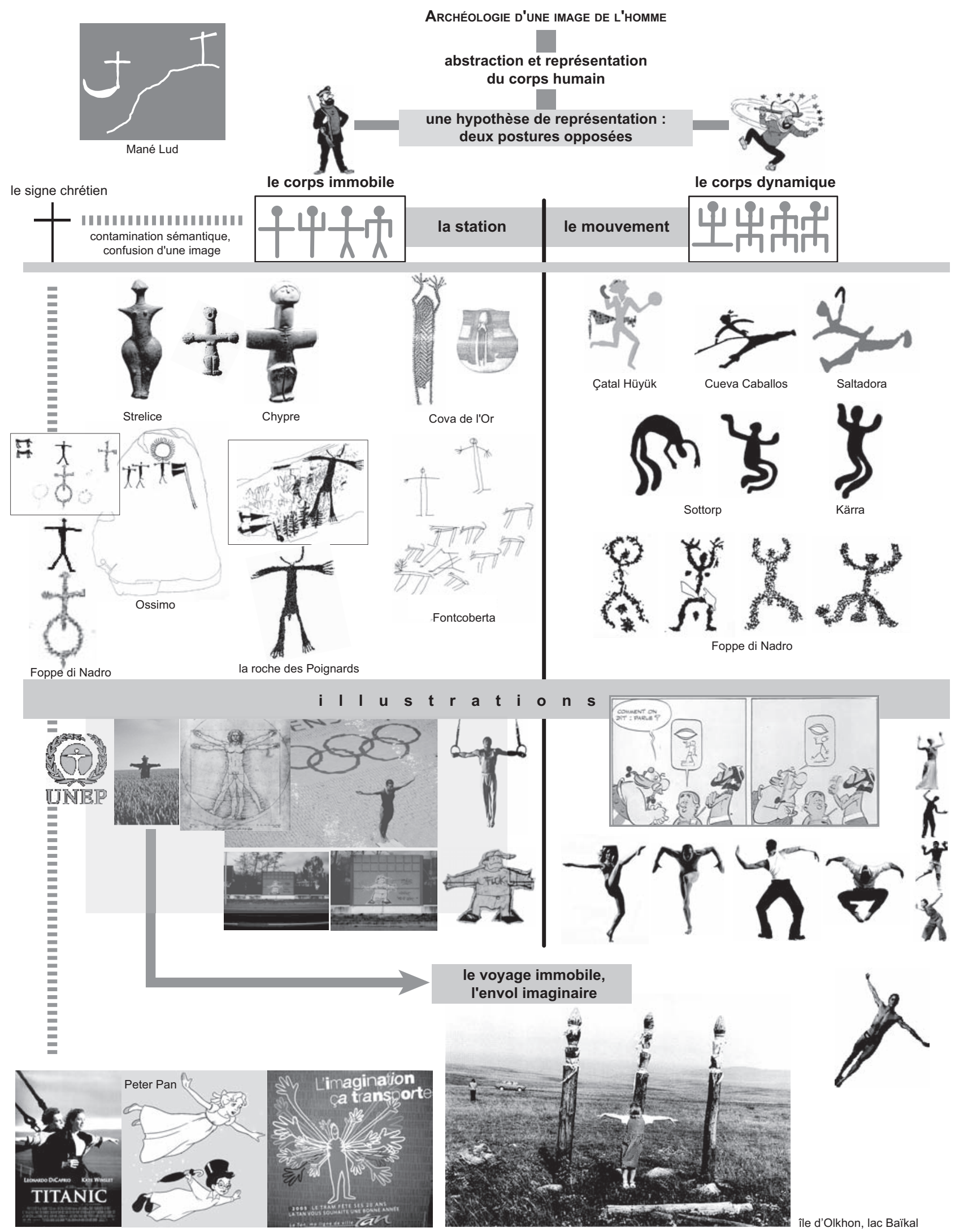
- l'orientation du sujet animal par rapport à lui-même ;

- l'orientation spatiale par rapport aux points cardinaux terrestres;

- l'orientation temporelle, enfin, par rapport aux points cardinaux célestes.

La croisée de ces deux derniers axes majeurs réalise la croix d'orientation totale (Leroi-Gourhan, 1964, p. 162 ; Radkowski, 2002, p. 151).

Voici un point de rupture du temps et de l'espace, si bien compris qu'un motif crucifère comme la croix cerclée a représenté, pour les Irlandais de l'époque carolingienne, une synthèse intime et parfaite du christianisme et de la tradition antérieure, correspondance étroite des anciennes conceptions «celtiques» et de données ésotériques chrétiennes (Chevallier, Gheerbrant dir., 1969) (fig. 5).

En somme :

- le signe en croix inscrit sur les stèles du Mané Lud n'est pas un outil mais la représentation d'un anthropomorphe de type statique, du type «crucifié ", du type du «Christ-Homme » protégeant de son arme ou de son aile la baie de Saint-Nazaire, de Lisbonne ou celle de Rio de Janeiro (fig. 5), une posture à l'image du plongeur d'élite fixé au-dessus de l'eau, tout comme salue le gymnaste face à la foule, ou le sportif aux pieds joints et aux jambes raides qui agit par procuration devant la foule attentive, et dont

Fig. 6 - Archéologie d'une image de l'homme selon un principe d'opposition fondamental: le capitaine Haddock dans Les Aventures de Tintin, Le Crabe aux pinces d'or (Hergé, 1953, (C) Hergél Moulinsart, 2006) ; statuettes de Strelice, République tchèque (d'après Gimbutas, 1989) et Chypre (d'après Karageorghis, 1976 ; Crewe et al., 2002), Cova de l'Or, Espagne (d'après Marti Oliver, 1990) ; gravures de Foppe di Nadro, Italie (d'après Sansoni, Maretta, 2002), Ossimo, Italie (d'après Anati, 1996), la roche des Poignards, Hautes-Alpes (Gruppo ricerche cultura, Montana, 1994, http://www.rupestre. net/archiv/index.htm), Fontcoberta, Espagne (d'après Abelanet, 1990), Catal Hüyük, Turquie (d'après Mellaart, 1967), grottes de Cueva Caballos et de Saltadora, Espagne (d'après Adams, 1984); gravures de Bohuslän, Suède (d'après Coles, 2004) et Foppe di Nadro, Italie (d'après Priuli, 1979) ; logo de United Nations Environment Program ; épouvantail, Suisse (http://www.epouvantail.ch/album_01/); corps humain du traité d'architecture de Vitruve, illustré par Fra Giocondo en 1551; site officiel des jeux olympiques d'Athènes; tags sur un mur de l'avenue Préales à Rennes, 2004 (cliché: S. Cassen, CNRS); Astérix et Cléopâtre (d'après Gosciny, Uderzo, 1965 (C) éd. Dargaud et l'autorisation des éditions Albert Renée) ; photos de danseurs (http:// www.zefa.fr/index) ; affiche du film Titanic (http://fr.allposters.com/) ; extrait de Peter Pan (@ Disney Prod.) ; affiche de la société de transport nantaise TAN (2004); poteaux d'attache pour "chevaux célestes", groupes bouriates, île d'Olkhon, lac Baïkal, Sibérie (d'après Doury, 1999). les anneaux latéraux de métal ne modifient en rien l'attitude immobile et si faussement reposée, le simulacre d'une station au paroxysme de la contraction des muscles du mouvement, le paradoxe d'un mouvement statique ;

- ce type statique nous est désigné par les premiers écrits européens aux commencements héroïques. Ilos, fils de Trôs, fonda une ville qu'il appela Ilion (Troie), priant Zeus qu'un signe se manifestât à lui : ce fut la "petite Pallas ", tombée du ciel, posée devant sa tente : c'était une figure aux jambes jointes et aux pieds non séparés, aux bras écartés, pour qui Ilos bâtit un temple et constitua un culte. Telle est la légende du «Palladion» (Dumézil, 1985, p. 39) ;

- voici la posture divine, comme le portrait de l'acteur de cinéma saisi par le célèbre studio d'Harcourt, comparé par R. Barthes (1957, p. 24) à un dieu puisqu'il ne fait jamais rien, puisqu'il est saisi au repos, car marcher est peut-être - mythologiquement - le geste le plus trivial, donc le plus humain. Tout rêve, toute image idéale, toute promotion sociale, ajoute-t-il, suppriment d'abord les jambes, ou les fusionnent, les fondent sous le portrait, sur un cheval ou dans une automobile ;

- une posture pour désigner un être distingué dans toute l'Eurasie, le chamane de Sibérie, qui assure cette médiation avec la Surnature (Hamayon, 1990), le seul à pouvoir se munir des ustensiles magiques à l'image du tambour qu'il " chevauche ", un tambour couvert de pictogrammes agencés pour assurer la division du monde et dont nous avons tiré profit par l'intuition travaillée dans l'interprétation du décor céramique de type castellic (Cassen, 2000d). Ces tambours sont conservés dans les musées régionaux (voir la collection ancienne de la ville de Biysk, Russie), que l'on sait représentés à l'identique, avec minutie, parmi gravures et peintures pariétales conservées (fig. 7), et dont la présence même permet de séparer dans la composition d'une stèle comme celle de Karakol dans l'Altaï (Kubarev, 2002), d'une part les hommes mobiles aux membres fléchis, d'autre part le seul être à jambes jointes et bras écartés et tendus, lui seul porteur du tambour ${ }^{19}$.

De telles scènes ont également été inscrites sur les parois de ces montagnes (Gorniy Altaï) au cours des derniers

19. Cette distinction faite à partir de la posture du chamane, que nous opposons aux autres représentations anthropomorphes, ne doit pas faire oublier que, par essence, cet homme ou cette femme bougent: la racine sam-, d'où vient saman, contient dans les langues toungouses une idée de mouvement du bas du corps - " remuer les pieds, bondir, sauter»-qui évolue dans le sens de «s'agiter». Le sens de «bondir, sauter, remuer les pieds, trépigner » figure aussi dans l'étymologie de verbes voulant dire «chamaniser» ou désignant une conduite rituelle chamanique dans d'autres langues de Sibérie (Hamayon, 1990). 


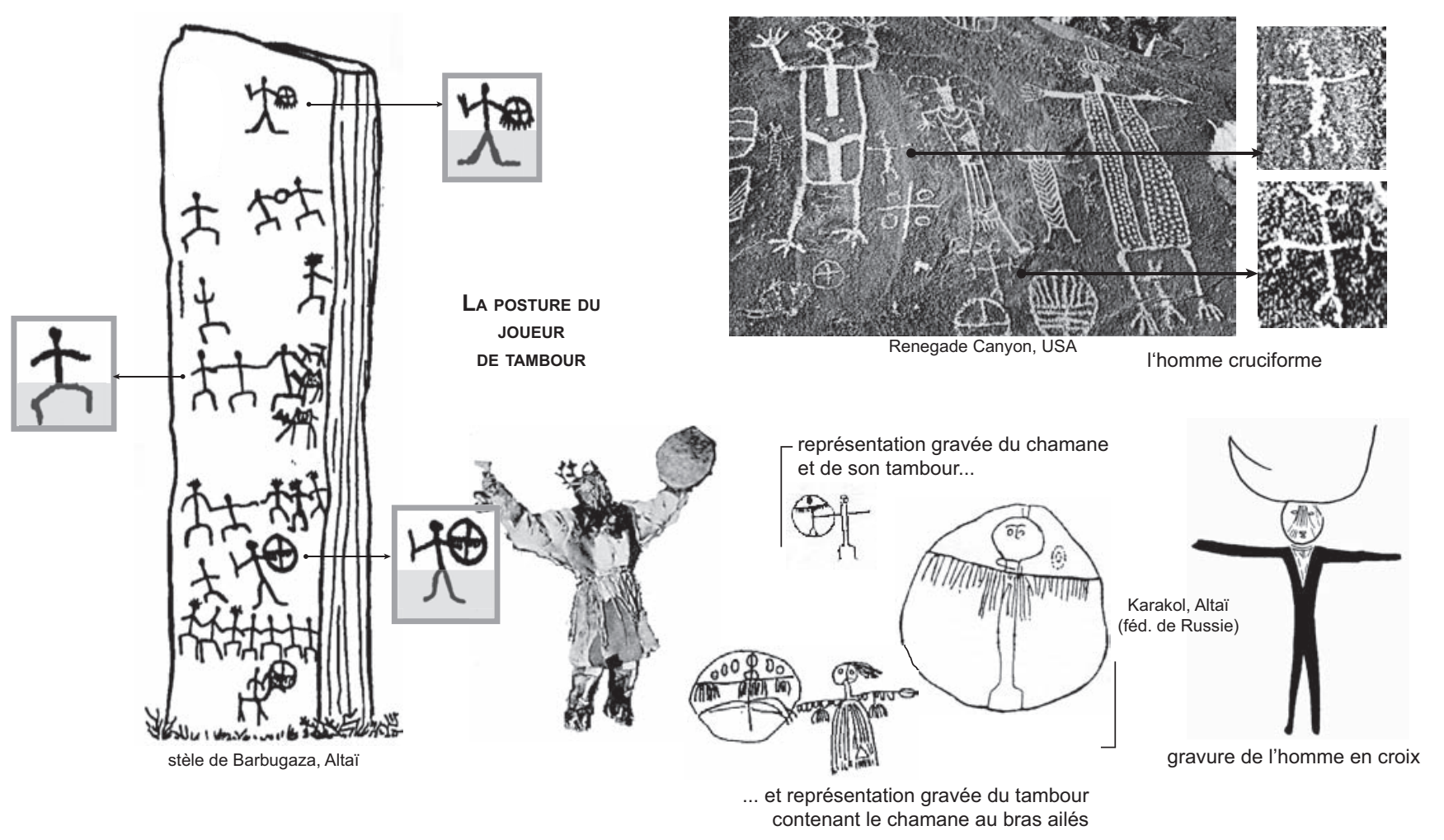

Fig. 7 - Représentation du porteur de tambour (chamane) en Sibérie et figurations d'anthropomorphes cruciformes en Amérique du Nord: stèle de Barbugaza, Altaï russe (d'après Kubarev, 2002) ; représentations gravées du chamane et de son tambour, et gravures de tambour sur paroi rocheuse à Karacol, Altaï russe (d'après Kubarev, 1988 et 2002) et Shalboby, Russie (d'après Okladnikova, 1996) ; chamane Yakout (photo: Klemenc, 1903, musée d'Ethnographie à Saint-Pétersbourg) ; représentations de personnages bras en croix ou bras levés asymétriques et abstractions cruciformes de personnages tridactyles à Renegade Canyon, USA (d'après Grant, 1983).

siècles, au sujet desquelles les relations ethnographiques nous informent sans ambiguité sur la nature du personnage représenté, statique et muni du tambour, parfois de son battoir, un tambour lui-même gravé, restituant en miniature et comme en abîme, sur sa surface circulaire, le «guérisseur " les bras en croix (Kadikov, 2005, p. 11), les bras ailés pour un envol (fig. 7). Nombreux sont en effet les témoignages où le « vol » chamanique s'apparente à celui d'un oiseau, ce que traduisent d'ailleurs certains costumes aux manches garnies de rémiges d'aigle, un vol planant matérialisé par la posture du corps aux bras écartés durant telle ou telle phase de la séance (Beffa, Delaby, 1999). Une posture que traduisent les cerfs-volants maoris de Nouvelle-Zélande (fig. 4), hommes-oiseaux parfois limités au seul objet cruciforme, lancés vers le ciel au premier lever des Pléiades signalant la fin de l'année (Best, 1934 ; Maysmor, 2001).

C'est une figure que les travaux recensés à partir des années 1870 dans l'ouest de la France ne parviennent pas à définir et à interpréter, arguant parfois de la forme en croix pour assurer l'âge gaulois des dolmens (Galles, 1873), tandis que les rapprochements établis dès cette époque entre Morbihan et un site aussi emblématique que Méniscoul à Piriac-sur-Mer (Loire-Atlantique) poussent les tenants du signe préhistorique à l'intégrer dans un courant de diffusion allant de l'Espagne à l'Irlande, mais sur la foi de registres très contestables (Breuil, 1934-1935). L'âge de cette dernière dalle, chargée en cruciformes, balance ainsi entre Histoire et Préhistoire (G. Bellancourt, en 1977, conteste que ces cruciformes aient pu être réalisés avec un instrument métallique), au point que les analyses techniques les plus récentes ne parviennent toujours pas à trancher sur l'ancienneté de ces signes, en raison des possibles phénomènes érosifs propres au domaine littoral, occasionnant avec le temps des tracés par trop indifférenciables (Mens, 2003). Et la même dalle guérandaise n'en finit pas de stimuler l'observateur, renvoyant encore aujourd'hui vers l'Irlande quand un spécimen semblable lui est comparé à Clonfinlough (Shee Twohig, 2002), sans que la minutieuse description des faits et du contexte ne permette 
de trancher sur la période d'exécution, l'auteur favorisant cependant l'âge historique des croix gravées sur la foi d'un monastère édifié à $3 \mathrm{~km}$ de là.

Une indécision chronique, finalement, dont le Mané Lud ne peut se satisfaire, car la liste argumentaire assure ici la contemporanéité de toutes les composantes :

- par la protection assurée depuis 6000 ans au sein de l'architecture de la chambre, relativement intacte en son cairn isolant, qui élimine le processus brouilleur de météorisation "postmégalithique ";

- par l'homogénéité du traitement technique des différentes gravures - toutes réalisées par percussion lithique que rien ne distingue à l'œil nu, en photographie et en estampage ainsi qu'au toucher, sinon les altérations différentielles dues à la météorisation "prémégalithique » affectant certaines parties de la dalle (Cassen et al., 2005) ;

- par l'effacement général des gravures sur la stèle $n^{\circ} 21$, quasiment invisibles à l'œil nu malgré un éclairage adéquat, qui traduit localement un phénomène érosif antérieur au recyclage de la stèle fonctionnant en second emploi en tant qu'orthostate au sein du tombeau, un estompage qui s'applique indifféremment à l'ensemble des tracés, croix comprises ;

- enfin par l'intégration parfaite du signe dans les compositions reconnues (voir infra, p. 225 : le signe dressé à l'extrémité d'un bateau) qui élimine l'hypothèse contradictoire d'un ajout parasite, les superpositions de tracés n'étant par ailleurs aucunement attestées sur le site.

Le cruciforme du Mané Lud est la représentation d'un personnage en position arrêtée, jambes fusionnées et bras écartés dans la posture de l'homme total, certes, mais enfin dans l'attitude la moins «narrative » qui soit; peut-être la plus exceptionnelle qui soit.

\section{LA CROSSE}

La crosse - quelles que soient les variations morphologiques régionales de l'instrument sur lesquelles nous ne porterons pas l'accent en ces lignes - est un signe qui n'a pas fait l'unanimité chez les archéologues, comme le fut dès l'origine son parèdre, la hache.

Résumons en premier lieu ce qui fut par ailleurs étendu (Cassen, 2000e), en reprenant les définitions ou acceptations convenues, avant de pouvoir déshabiller le signe des pelures de son histoire savante et reconstruire une image cohérente avec le contexte proposé ; les relations de voisinage avec les autres signes du Mané Lud seront ensuite exposées, les associations ordonnées et les compositions orientées.

\section{DÉCONSTRUCTION DES TERMES EN USAGE : UN INSIGNE PACIFIQUE, PACIFIÉ}

Un contexte agropastoral prévaut en tout premier lieu lorsqu'une faucille est reconnue dans l'objet, qu'il s'agisse de la gravure morbihannaise, du bijou bulgare à Varna (Ivanof, 1991, p. 197) ou de l'homme hongrois assis et modelé de Szegvár-Tüzköves (Kalicz, 1970, p. 44). Le bâton de berger est encore plus souvent revendiqué en Bretagne ainsi qu'en péninsule Ibérique (Bradley, 1989, p. 70 ; Calado, 1997 p. 295 et 2002, p. 29) par allusion, volontaire ou parfois inconsciente, à la hiérarchie catholique, dès lors que l'ancien et probable objet est lui aussi reconnu en tant qu'insigne de statut, à l'image de la crosse épiscopale; et la canne de l'évêque étant acceptée comme l'illustration de l'assise spirituelle, dans un registre iconographique en paix avec le troupeau des ovins, donnant dans l'immuable, la crosse néolithique est dotée des mêmes qualités par l'illusion métaphorique. Ce sont d'ailleurs des brebis ou des chèvres que l'on trouve sous notre signe sollicité (Tresset 2005, p. 278).

Un contexte autoritaire est implicitement compris, et ce depuis le $\mathrm{XIX}^{\mathrm{e}} \mathrm{s}$, quand les rois de l'Égypte ancienne sont convoqués au chevet du signe interprété (heqa), pour lui procurer par capillarité un peu du prestige d'un passé glorieux; l'emblème évasif du roi est encore peu ou prou sous-jacent dans l'allusion faite au fameux lituus des étranges Étrusques ou à la crosse en bois et en or des plus lointains Hittites dont les souverains sont bel et bien porteurs (Alps, 1947).

Un contexte religieux est en conséquence le terme moyen et sage, pour ne pas dire fourre-tout, qu'il est d'usage d'accepter si l'on ne veut accréditer ouvertement telle ou telle de ces interprétations par trop avancées et mal soutenues ; le signe est celui de la «divinité » plus ou moins sexuée, il est donc d'essence divine, son caractère est par là même religieux, et le cercle se referme...

\section{RECOMPOSITION}

- l'association avec un objet violent assure le premier terme récurrent en Bretagne, et le mieux vérifié sur stèle ; la hache est l'arme/outil avec lequel la crosse se présente en position verticale stabilisée (voir le site de Runesto à Carnac, le comparer avec le Mané Lud : Boujot et al., 2000) ;

- une mise en situation d'affrontement ressort d'une composition liant la crosse - cette fois en position d'instabilité, penchée par rapport à l'axe longitudinal de la 
stèle - aux animaux des stèles du Bronzo et de la Table des Marchands : la partie courbe de l'objet est dirigée vers le cachalot et l'oiseau (Cassen, Vaquero, 2003) ;

- la crosse est un objet reconnu en contexte archéologique de la Préhistoire récente de l'Eurasie, aussi bien en bois (Brøndsted, 1957), en os, en pierre (Leisner, Leisner, 1956) qu'en métal (Smirnov, 2004) ;

- sa représentation aux bras des humains est également attestée dans le corpus pariétal " préhistorique ", mais selon deux termes très opposés dans une alternative cohérente (Cassen, 2007) : aux mains de chasseurs courant le gibier (cervidés, léporidés) et aux mains de personnages hiératiques, immobiles ;

- une constellation d'images et de symboles qui traduit la longue histoire du passage d'une arme de jet, d'emploi universel, vers l'insigne mythologique, insigne de statut, voire instrument de divination, dans des temps et des lieux différents (roi hittite, devin étrusque, voir Piel, 2000 et 2003; chamane de l'Altaï, medecine-man des populations de l'Arizona, etc.) ;

- une arme qui n'a jamais fait couler le sang et qui, par cette qualité même, permet peut-être de comprendre sa longévité, sa répartition, en jouant de surcroît sur la nature de l'objet composite (frappe, crochet, canne-support, etc.) et la polysémie conséquente du signe ;

- un objet « totémique » et un signe, enfin, qui, ensemble par leur ambivalence essentielle, jouent pleinement, sur fond d'altérité, aux mains des personnages assurant le passage des états antagonistes dénotant la situation de crise, de danger autant que la force calmée, maîtrisée ;

- un signe, par conséquent, dont le doublement par opposition des parties actives, en Bretagne comme au Portugal (fig. 13), devra se concevoir dans ce rapport à l'altérité.

\section{LE CONTEXTE GRAPHIQUE DU MANÉ LUD}

Pour mémoire, et prendre date, les associations de signes pourront être ordonnées comme suit :

a) le signe unique est en association stricte :

- avec le motif quadrangulaire, en le flanquant latéralement (stèle $\mathrm{n}^{\mathrm{o}}$ 21),

- avec la hache, leurs parties actives affrontées (stèle $n^{\circ} 21$ ),

- avec le «pectiniforme" ou bateau: en avant de la figure, ou en fusion avec elle (stèle $\left.n^{\circ} 16\right)$;

b) le signe double, en symétrie miroir et les parties actives affrontées, surmonte une autre composition symétrique de trois signes « cornus " superposés (stèle $n^{0} 2$ ) ;

c) le signe double, parties actives non affrontées, est en situation d'isolement tout en marquant une région de partage ou de transition à la surface de la pierre (fissure horizontale sur la stèle $\mathrm{n}^{\circ} 21$ ).

$\mathrm{Au}$ surplus, le signe s'oriente dans chaque scène en suivant différentes contraintes :

d) il est vertical, orienté selon l'axe longitudinal de la stèle-orthostate (stèles n ${ }^{\text {os }} 16$ et 21 ) ;

e) il est oblique en fonction du motif voisin (quadrangulaire) qui, lui aussi en position penchée par rapport à l'ensemble des autres signes (stèle $\mathrm{n}^{\mathrm{o}} 21$ ), dicte son orientation ou subit cette orientation ;

f) il est oblique, subissant la forme préexistante d'une fissure dans la roche qui dicte l'implantation et l'orientation.

Cette liste prendra vie dans l'article sur Le Mané Lud en mouvement.

\section{LE CORNU}

\section{UN SIGNE INCONTESTÉ}

Avec la hache et la crosse, le " cornu " est historiquement le signe reçu par excellence, tant l'évidence graphique impose aux archéologues, depuis le $\mathrm{XIX}^{\mathrm{e}}$ s., sa loi de la reconnaissance visuelle spontanée.

Ce signe témoigne d'un culte taurin, dont l'origine probable est peu ou prou acceptée comme provenant de Méditerranée orientale, avec au point de départ J. Déchelette (1908-1928) qui, reconnaissant pourtant dans les monuments mégalithiques occidentaux ce qu'il y a de "spontané, d'original et d'indigène en eux » - en s'opposant ici au «mirage oriental » qu'il dénonce à juste titre, en accord pour une fois avec S. Reinach (1893) - ajoute qu'avec les chambres à galerie pénétrait en Gaule, aux côtés du taureau, l'image d'une divinité primitive, également originaire du bassin oriental de la Méditerranée, «divinité funéraire », « idole féminine », « gardienne des sépultures », autrement dit un vocabulaire, un animal et une aire géographique qui seront sans bouleversement aucun reproduits tels quels jusqu'à nos jours.

Dès lors, et sans discontinuité, ce signe cornu symbolise invariablement les activités agropastorales (Tresset, 2002, p. 47 et 2005, p. 277 ; Laporte, Le Roux, 2004 ; Marchand, Tresset, 2004, p. 275), celles qui s'accordent avec la définition usuelle du Néolithique; ou, avec plus de raffinement dans l'analyse, rappelle par son inscription dans la pierre l'obtention du prestige par le biais du sacrifice des animaux domestiques, dont on expose et superpose les bucranes, dans le cadre compétitif reconnu à une société à forte hiérarchie sociale (Defaix, 2001). 


\section{UN SIGNE À L'INTERPRÉTATION CONTESTABLE}

Un bucrane est constitué, comme son nom l'indique ${ }^{20}$, du frontal ou, mieux, du crâne de l'animal, et des chevilles osseuses solidaires de la calotte crânienne, elles-mêmes supportant les cornes véritables, l'ensemble de ces composantes devant être produit pour signifier la bête cornue à l'heure de l'abstraire, ce que ne restituent pas les gravures du Mané Lud dès lors qu'une comparaison terme à terme est menée avec les cornus vrais dans des contextes distincts (Cassen, 2005).

Face à l'acceptation unilatérale et univoque des archéologues, il est bon de rappeler qu'une autre lecture spontanée est possible, et concurrente, et tout aussi légitime en ces questions de représentations passées d'une société «orale », et qu'elle désigne les oiseaux comme une famille de prétendants tout aussi acceptable (voir notre interrogation publique dans Cassen, 2005).

Une catégorie animale extraordinairement présente sur la stèle du Bronzo, dans la même commune, intégrée à une composition figurative dont l'armature «structurale " est cohérente et partagée avec ses voisines (Cassen, Vaquero, 2003); une stèle où un oiseau est affronté à une arme comme le fut son parèdre marin, le cachalot, retrouvé à son tour sur d'autres stèles mais caché sous des formes variées par l'effet d'un processus d'abstraction poussé, et qui, par cette réplication même au sein d'un dispositif mythico-religieux, impose de rechercher pour l'oiseau d'autres solutions graphiques; d'autres formules qui, comme il est probable, seront des échos simplifiés du modèle au point qu'ils ne permettront pas cette reconnaissance par les archéologues s'ils demeurent privés de l'idée en question.

Un oiseau dont la forme à reproduire au loin dans le ciel n'est pas une histoire passée, inaccessible et révolue, puisque nous la retrouverons intacte dans l'univers cognitif de l'homme actuel, ou dans un temps plus lointain chez les «sauvages » d'Amérique et de Scandinavie.

Un oiseau, enfin, représenté au Mané Lud dans une position le faisant surmonter, survoler systématiquement le «pectiniforme » (stèles $\left.n^{\text {os }} 6,16,21\right)$, le «bateau» de G. de Mortillet.

20. Emprunté au bas latin bucranium, "tête de bœuf» (Corpus Inscriptionum Latinarum), le bucrane est en architecture gréco-romaine ce motif ornemental qui se composait d'une tête de bœuf décharnée, aux cornes ornées de guirlandes et de fleurs, et qui fut utilisé pour assurer la décoration des frises de temples et d'autels.

\section{LES MESSAGERS DE L'ARCHE}

Une étape supérieure de la reconnaissance a résidé dans l'identification de l'oiseau mis en scène. Puisqu'un cachalot fut signifié, un bovin et un ovin distingués, le volatile gravé sur la stèle du Bronzo devait, suivant cette nature cohérente, pouvoir être désigné par les caractères visuels de son espèce.

L'étude minutieuse des tracés a permis une sélection progressive des images, au point d'aboutir à une seule confrontation opposant les colombidés (mais sans les tourterelles, le pigeon colombin et le pigeon biset) aux corvidés (d'où furent évacués la pie, le geai et le cassenoix moucheté), couple mythique par excellence qu'une longue histoire réunit dans des récits divers, héroïques ou fondateurs, autour des concepts antinomiques que sont la guerre et l'amour, le sec et l'humide, la clairvoyance et l'aveuglement, et ce pour deux bêtes communes placées par les biologistes du comportement aux deux pôles extrêmes de «l'intelligence » des oiseaux (Cassen, 2005, p. 314).

Une ambiguïté poussée à un point inimaginable puisque le nom de la stèle renvoie en breton au nom probable du corbeau (Men Bran Sao), un toponyme qui ne peut remonter au-delà du $\mathrm{V}^{\mathrm{e}}$ s. apr.J.-C., date d'arrivée des locuteurs utilisant une forme de celtique étroitement apparentée au cornique et au gallois; un toponyme qui, bien entendu, devait avant cela être vivant dans le langage des Vénètes. Mais, étrangement, pour un oiseau que nous identifions au pigeon ramier en se fondant sur une somme de détails graphiques. Un oiseau qui était, enfin, invisible à ceux qui le dénommèrent ou le traduisirent ainsi...

À ce jour, nous proposons de reconnaître dans l'oiseau du Bronzo un pigeon ramier à la bande alaire blanche, le gibier le plus chassé de l'ouest de la France; un oiseau migrateur, atlantique et ibérique puisque résidant en hiver, pour une très large majorité, entre Portugal du Sud et Basse-Andalousie, après un survol européen les conduisant à se regrouper au sud des Landes, le long de l'océan, avant leur passage des Pyrénées les plus basses.

Un pigeon en bande que peut alors restituer la stèle 17 du Mané Lud.

\section{LE BATEAU : PEIGNE ET GRAFFITI}

D’une acceptation déjà ancienne dans l'histoire des recherches (Mortillet, 1894), ce signe sera sévèrement remis en cause par J. L'Helgouac'h (1998), également rejeté par 
L. Laporte et C.-T. Le Roux (2004, p. 106), qui ne reconnaissent plus de bateau - et encore moins son équipage... - dans le «pectiniforme » de G. de Closmadeuc. Mais, à ce jour, aucune autre interprétation n'a pu occuper le terrain libéré par cette ultime dénégation.

Reprenons l'exercice et tâchons de préserver une cohérence d'ensemble au possible contexte, à la forme enregistrée et aux illustrations pouvant témoigner de ce choix précoce en faveur d'une embarcation.

Quel contexte pour un bateau?

Le milieu d'implantation du signe en Bretagne renvoie encore une fois à l'océan par sa répartition tout au long du rivage (Shee-Twohig, 1981).

L'histoire du développement de ce premier Néolithique passe par un ensemble de témoins dont l'essentiel des composantes à ce jour reconnues sont en rapport avec la mer :

- d'une part, des groupes de chasseurs-pêcheurs occupant densément la zone côtière, à une latitude où la très favorable biodiversité est mise à profit dans les stratégies de subsistance (Péquart, Péquart, 1954 ; Gouletquer, 1991a) ;

- d'autre part, des groupes appartenant à la vaste famille méridionale de l'Épicardial, qui prolongent la remontée des influences du Cardial par l'Ebro (Rojo Guerra, Kunst, 1999), tandis que d'autres voyageurs aux traditions semblables arrivent par voie maritime au long des côtes portugaises (Zilhão, 1998), des groupes d'un Épicardial identifié sur la bande littorale aquitanienne, puis charentaise (Laporte, Picq dir., 2002), essaimant selon un processus de déplacement dont l'histoire de la diffusion des hommes en Méditerranée a pleinement démontré la réalité : les voyages hauturiers (Cherry, 1981) ;

- enfin, de la synthèse opérée sous les avancées du Néolithique «vrai» en provenance du Bassin parisien, émerge une entité culturelle originale en Armoriquesud durant le Ve millénaire, le Castellic, qui emploie avec constance des coquilles marines dans la mise en place du système décoratif, coquilles dont la dominante bien spécifique (Gibbula magus) est peut-être choisie pour ses qualités iridescentes (Cassen, 2000d).

Le mythe figuré sur les stèles - une représentation du monde et sa division classificatoire - est construit pour une part sur des animaux sauvages venant d'un au-delà des mers :

- le cachalot dont l'actualité récente donne l'occasion irrépressible à tout un chacun de venir se recueillir devant la bête fabuleuse, échouée (à Plozévet, Finistère, en août
2004, puis encore, si proche du Mané Lud, sur une plage en presqu'île de Rhuys, en octobre 2004) ${ }^{21}$;

- le pigeon ramier, lui aussi migrateur, qui annonce le printemps pour certains mais qui, surtout, en bandes innombrables, arrive de toute l'Eurasie pour se regrouper à l'automne au-dessus des rivages de la France de l'Ouest et aller séjourner au sud du Portugal et vers l'Andalousie atlantique (Cassen, 2005).

La mer est contemplée, observée, parcourue, traversée, combattue, intégrée au système de croyances.

Qu'une embarcation soit un outil de la survie alimentaire ne suffit pas à la représenter; qu'elle soit un moyen de communication entre les hommes de la Petite Mer n'est pas davantage le motif puissant de la figurer, mais qu'elle porte le héros fondateur, civilisateur, ordonnateur du monde, ou demeure le vecteur du Voyage vers les Îles Fortunées où les morts reposent alors en toute félicité auprès des possibles ancêtres, alors le bateau, dans ce contexte marin, et seulement celui-là, prendra forme impérative dans la narration et se cristallisera en un symbole immédiatement perçu.

Précisons la forme en question. Quelle est la ligne graphique directrice?

Sur les orthostates de la tombe, deux formes visibles: d'une part un tracé limitant un croissant horizontal (orthostate 21) ; d'autre part un seul trait large horizontal aux extrémités relevées (orthostate 6, fig. 9). On note en ce dernier cas l'adjonction de cinq à six traits verticaux et parallèles, dont l'un dépasse systématiquement en longueur ses voisins. Il est alors placé au centre (une fois), mais plus fréquemment aux extrémités du motif basal (trois fois).

Deux traitements techniques distincts affectent ces deux sous-ensembles, lignes normales entre elles :

- le premier décrit est dégagé par percussion, en creux dans la roche, et le profil en U de la gravure est habituel, attendu ;

- les segments verticaux sont par contre obtenus par des sortes d'incisions grossières qui ne déterminent pas de tracés francs ni de section en $\mathrm{U}$; symptomatiques du traitement au moyen d'un autre instrument de gravure, les extrémités supérieures sont difficiles à repérer par photographie car elles n'ont pas fait l'objet d'une percussion arrêtée, pointe arrondie, mais plutôt de tracés divergents, ne se recouvrant pas nécessairement. Ce traitement partagé, par traits verticaux parallèles, si particulier, est observable

21. Un grand merci à P. Gouletquer qui a recueilli les informations journalistiques sur ces échouages attirant plusieurs milliers de personnes, paralysant les routes départementales... 
également au Mané Rutual dans la même commune et pour un signe semblable (Cassen et al., 2005).

Arrivé à ce point de l'analyse descriptive et passée l'observation technique, il nous faut marquer une pause dans ce processus de reconnaissance appliqué au Mané Lud, en sollicitant l'appui d'un autre signe capable de nous assurer une progression tangible de l'argumentaire.

D’une façon plus générale en Armorique, il est en effet nécessaire de réunir en une même catégorie graphique ces deux figures distinguées par les archéologues: d'une part, celle que nous venons de décrire sur l'orthostate $n^{\circ} 6$, qui est « croissantiforme ", mais à base très aplatie, parfois surmontée de traits verticaux parallèles ; d'autre part, cette autre figure en forme de croissant elle aussi mais plus franchement « lunaire » par sa convexité régulière (ex. : la Table des Marchands, dos du chevet).

Une même ligne directrice relie ces deux silhouettes qui ne furent, à notre connaissance, jamais comparées (fig. 8) ; une trop claire superposition des formes qui pousse à les réunir et empêche de les éloigner l'une de l'autre, d'autant mieux que des étapes intermédiaires assurent la continuité iconographique entre les extrêmes (ex.: Kermaillard à Arzon, Morbihan). Ce rapport analogique immédiat impose de les traiter simultanément pour mieux comprendre la dynamique des tracés du Mané Lud et mettre en œuvre ce test que nous tentons d'appliquer pour valider ou invalider l'interprétation en faveur d'un bateau.

Revenons à l'hypothèse de départ. Quels pourraient être alors, dans le monde des représentations à notre portée, les arguments figurés qui tendraient à corroborer l'idée d'une embarcation, d'un côté, celle d'un groupement de personnages, de l'autre, et comment leur réunion en une seule unité formelle serait recevable en tant qu'abstraction pure, comme image reproductible et solution graphique possible en des lieux et des temps séparés?

\section{LA COQUE}

Portons un regard indifférent sur les coques destinées à la mer et à la rivière.

La forme la plus courbe, en « croissant de lune » ou arc de cercle régulier, est indéniablement pensée et reproduite : elle est connue aussi bien dans l'univers médiéval européen que dans les figurations rupestres de Corée, aussi bien dans le registre de l'Égypte ancienne que dans les symboles pascuans du Pacifique.

Elle peut être isolée, c'est-à-dire vide, ou surmontée de personnages et de structures.
La forme aplatie allongée, dite «corniforme", est en réalité la plus fréquemment inventoriée et également la plus souvent montée dans les corpus à notre disposition : des graffitis normands historiques aux falaises des chasseurscueilleurs de l'Azerbaïdjan, des forêts du nord-canadien aux rochers de l'âge du Bronze scandinave.

Un tableau bref et comparatif, sans les assemblages montés, est par lui-même fort démonstratif (fig. 9).

\section{LES PERSONNAGES}

Partons de l'image réelle. Regardons l'horizon et cette embarcation de pêcheurs éloignée (fig. 9). Que retiendrat-on pour composer la scène ? Cette forme, assurément. Et en effet, comment vais-je abstraire, issue de ma mémoire visuelle, ce morceau flottant de matière habitée par des humains?

Tout d'abord, voyez comment les hommes sont embarqués et sculptés à Roos Carr (Angleterre), ou encore dessinés avec leurs rames par les graveurs algonquins (Canada), ou peints par l'enlumineur russe du Moyen Âge (fig. 9). Les hommes seront respectés dans leur nombre, leur allure et leurs proportions respectives, mais seulement tassés dans un espace réduit, miniaturisé, hors de proportion du contenant.

Alors seulement, dans un seconde temps, nous comprenons que l'on peut aussi simplifier ces personnages, progressivement les réduire, les ramener à la simple expression de cette fondamentale verticalité humaine, parfois confondue avec leurs rames sous-jacentes ${ }^{22}$, comme en miroir, comme les miroirs de leurs tracés d'hommes dirigés vers le haut. Ici en Alaska, vers 1880, ou dans les barques gravées et peintes de l'Égypte prédynastique, là sur les peintures paléolithiques du Gobustan en bordure de la mer Caspienne.

En résumé :

a) nous avons retenu un environnement spécifique, maritime ;

22. Et non l'inverse, à l'image des propositions de J. Briard qui, approuvant pourtant la représentation du bateau au Mané Lud, interprète les traits parallèles comme des « rames dressées " (Briard, 1995, p. 135) ; mais si la rame peut être rendue visible en position fonctionnelle, plongée vers le bas, tandis que le rameur reste caché dans le corps d'embarcation (par exemple, les représentations égyptiennes peintes sur céramiques), la valorisation verticale est universellement réservée aux hommes, voire aux hommes munis de leurs rames présentées, à l'instar du salut rituel ou de l'abordage d'un navire qui imposent ces levées de rames (voir les gravures interprétées dans ce sens, ou encore comme autant de torches, à Bro Utmark en Suède: Coles, 2004, p. 194), mais ces dernières, si nous les supposons isolées, et qui pourtant doivent être tenues, ne peuvent métaphoriquement surpasser l'homme qui les anime comme instruments. 

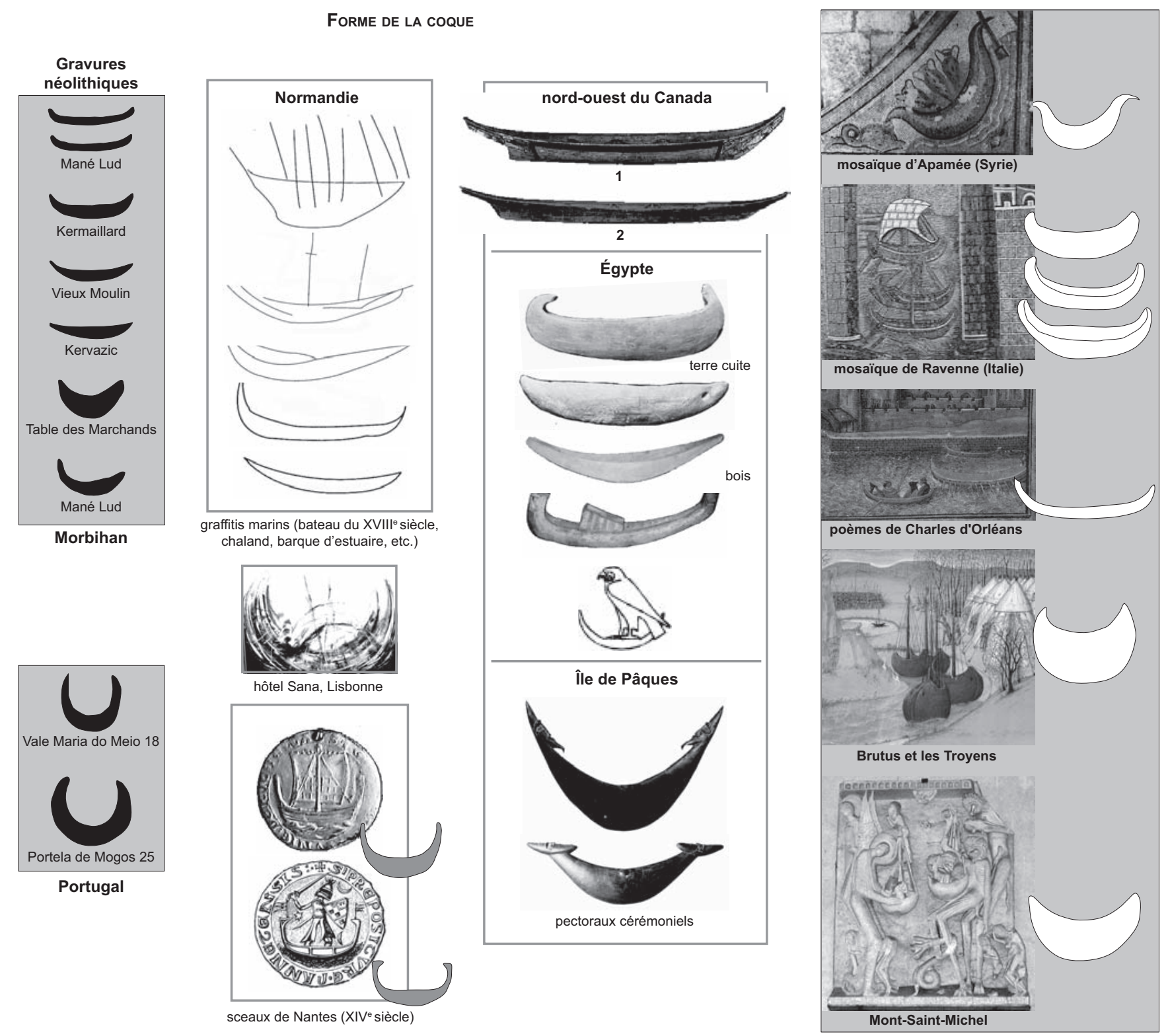

Fig. 8 - Étude sur la forme des embarcations : graffitis marins des églises du val de Saire, Normandie (d'après Carpentier et al., 2002) ; figuration abstraite et monumentale d'un navire sur les murs de l'hôtel Sana Metropolitan à Lisbonne, Portugal (cliché: S. Cassen, CNRS) ; sceaux du XIV e., Nantes, Loire-Atlantique; pirogues du nord-ouest du Canada: 1, pirogue de haute mer de type septentrional, îles de la ReineCharlotte, Colombie-Britannique, fin du XIX ${ }^{e}$ s. (http://www.civilization.ca/aborig/watercraft/wad03fra.html); 2, pirogue de haute mer de type salish de la côte Duncan, Colombie-Britannique, 1929 (http://www.civilization.ca/aborig/watercraft/wad03fra.html) ; modèles d'embarcations, Égypte prédynastique et dynastique (en argile cuite : d'après Petrie, 1920 ; en bois, musée du Louvre, cliché: S. Cassen, CNRS) ; hiéroglyphe : faucon dans une embarcation; pectoraux cérémoniels en bois représentant une embarcation, île de Pâques (d'après Maiani, Quer, 1996) ; mosä̈que du IV $V^{e} V^{e}$ s. apr. J.-C., Apamée, Syrie (d’après Kingsley, 1994) ; mosä̈que de Ravenne, Italie (d'après Kingsley, 1994) ; détail de l'enterrement de la tête de Bran à la Tour Blanche de Londres, 1444, manuscrit des poèmes de Charles d'Orléans (d'après Ashe, 1990); Brutus et les Troyens à l'embouchure de la Loire (d'après Ashe, 1990); la pesée des âmes au Mont-Saint-Michel, Manche (cliché: S. Cassen, CNRS). 


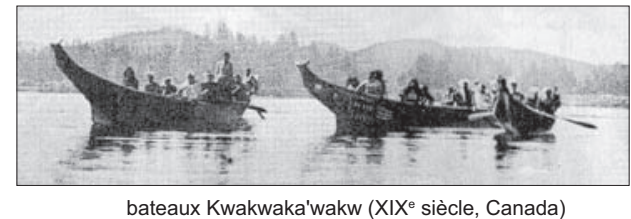

bateaux Kwakwaka'wakw (XIX'e siècle, Canada)

BARQUES MONTÉES

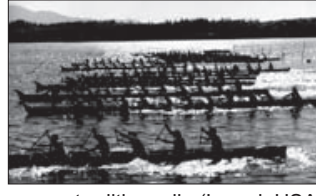

course traditionnelle (Lumni, USA

bateaux de haute mer
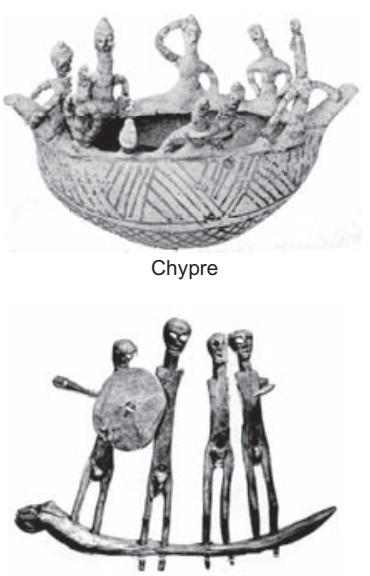

Roos Carr, Angleterre

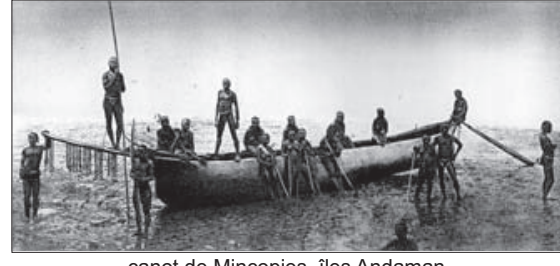

canot de Mincopies, îles Andaman

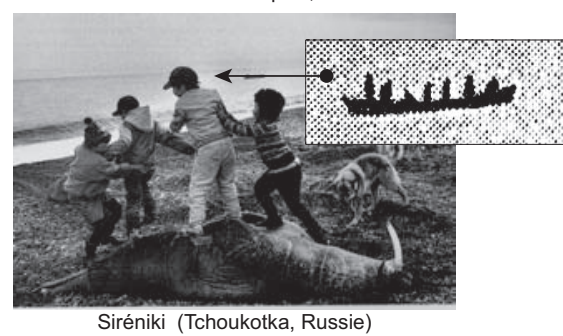

Siréniki (Tchoukotka, Russie)

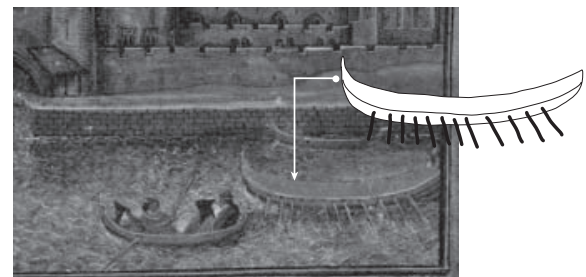

poèmes de Charles d'Orléans (1444)

Mané Lud

Russie

gravures

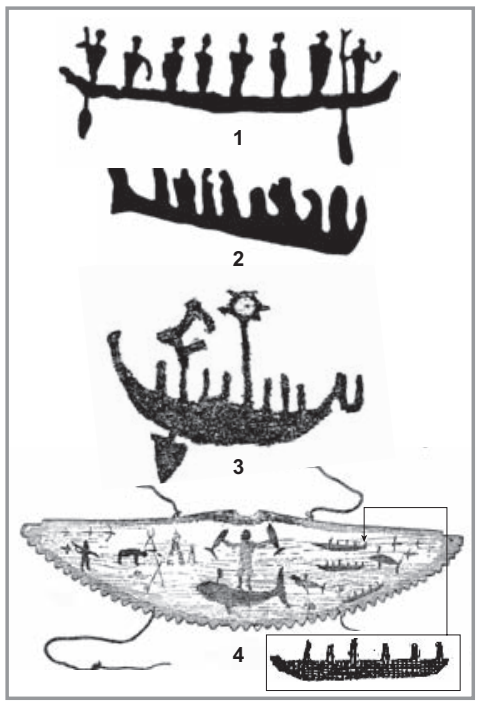

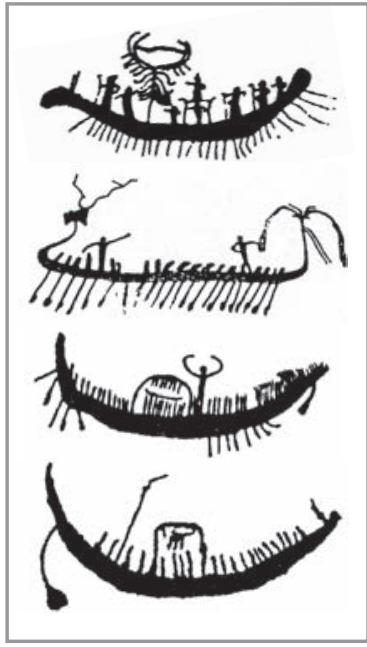

Nil, Égypte

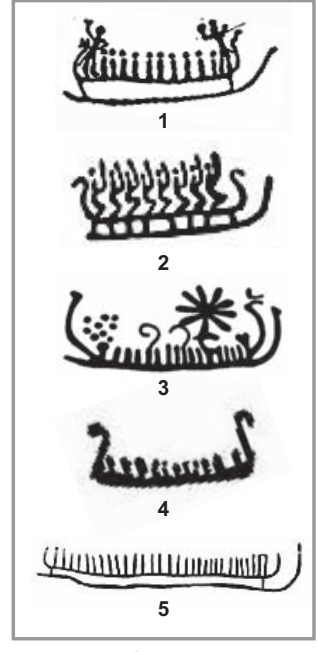

Suède

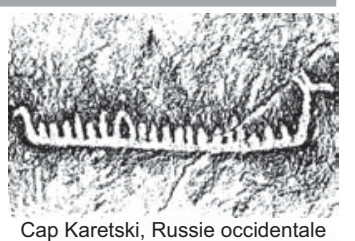

Cap Karetski, Russie occidentale

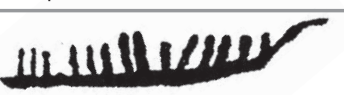

Tomskaya Pisanitsa, Russie centrale

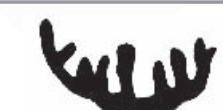

velletis

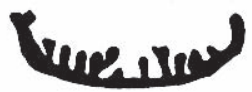

Sikachi-Alyan, Russie oriental

Canada oriental

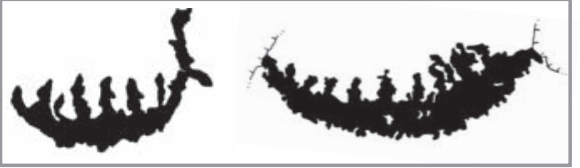

Bangu-dae, Corée

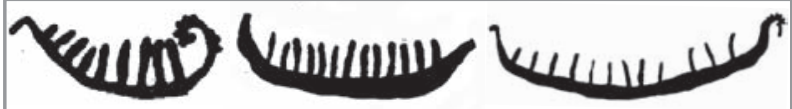

Gobustan, Azerbaïdjan

Fig. 9 - Représentations de barques montées, mer et rivière : bateaux Kwakwaka'wakw, Canada (@ musée canadien du Canot) ; course traditionnelle, Lumni Nation, USA; canot de Mincopies pour la haute-mer, creusé dans un tronc d'arbre, îles Andaman (d'après Verneau, 1949) ; illustration du voyage marin dans la Russie médiévale (d'après Ocetrov, 1985) ; bateau du Bronze moyen, avec personnages appuyés au bastingage et deux oiseaux perchés sur le rebord, Chypre (d’après Karageorghis, 1976) ; modèle en bois (Bronze final/Fer), de Roos Carr, Yorkshire, Angleterre (@ Hull Museum); chasse au morse, village de Siréniki, Tchoukotka, Russie (d'après Doury, 1999) ; détail de l'enterrement de la tête de Bran à la Tour Blanche de Londres, 1444, manuscrit des poèmes de Charles d'Orléans (d'après Ashe, 1990) ; Canada: 1, Pictured Lake, logo du musée canadien du Canot ; 2, oiseau Roc, Pontiac (Rajnovich, 1994) ; 3, Peterborough (@ musée canadien du Canot) ; 4, Point Barrow, Alaska (d’après Boas, 1883) ; barques sur le Nil, Égypte (relevés Winkler, in Müller-Karpe, 1968) ; gravures de Suède (http://europreart.net/preart.htm) : 1, Tanum, Sotetorp 1 ; 2, Askum ; 3, Skee, Massleberg ; 4, 5, Tanum ; cap Karetski, lac Onega, Russie (http://www.physics.helsinki.fi/whale/comics/poika/one13.html) ; Tomskaya Pisanitsa, fleuve Tom, Russie (d'après Martyinov, 2001) ; Sikachi-Alyan, fleuve Amour, Russie (d’après Ochibkina, 1996 ; Medvedev, 2001) ; Bangu-dae, Corée (d'après Sang Mog Lee, 2003) ; Gobustan, Azerbaïdjan (d'après Djafarzade, 1973). 
b) puis un contexte marin confortant, archéologique cette fois, où le coquillage marin participe à l'élaboration du message céramique, un contexte réfléchissant également la plus étonnante des figurations marines, le cachalot;

c) puis fut décrite l'ethno-archéographie des formes autrefois interprétées comme des coques de navires, des formes reconnues comme telles, sur d'autres supports, dans d'autres lieux et pour d'autres temps, mais au sein desquelles en réalité nous avons distingué deux variantes fondamentales divergeant par la convexité de leur tracé selon qu'elles soutiennent ou non d'autres figurations (des hommes) ;

d) des tracés verticaux, produits d'un second traitement technique qui les met à part et les désigne comme des humains, des hommes embarqués.

Finalement, des représentations comparables existent et nous serions inconséquent d'en priver le lecteur. De là une inévitable réaction en chaîne, quelques conséquences premières, consécutives à l'interrogation: que faire, en effet, des formes similaires rencontrées dans le registre armoricain? On l'a dit, le motif voisin de Kermaillard (Arzon), ou, plus loin, celui du Vieux Moulin (Plouharnel) en Morbihan ou encore de Spézet en Finistère, sont d'un commun accord compris comme la figuration d'un «cornu» (Le Roux, 1992).

- Mais, est-ce vraiment une corne?

Cheville osseuse ou corne véritable enveloppant cette cheville, l'objet doit être pointu à une extrémité, élargi à l'autre, et à ouverture circulaire. Objet-signe, la corne est «d'abondance» ou «de libation», voire instrument de musique, et la permanence de la tradition en faveur de ce contenant magique peut couvrir des millénaires en Europe du Nord, depuis la corne royale conçue en tant que récipient dans la souveraineté irlandaise (Dumézil, 1971) jusqu'aux objets identiques où coulent l'hydromel et la forte bière, et qui perdurent de La Tène récente jusqu'au XIII ${ }^{\mathrm{e}}$ siècle dans l'aire baltique (Bebre, 2003). Rien de cela n'est ici reproduit.

- Serait-ce alors un bucrane, qui autoriserait deux extrémités pointues pour signifier les parties efficaces de l'encornure? Mais le bucrane, dans tous les cas et par essence, contient le frontal qui réunit les chevilles osseuses et les cornes proprement dites (Cassen, 2005). Un frontal désigné par une tête, levant de facto toute ambiguïté, ou abstrait par le dessin d'un quadrilatère, d'un trapèze, etc., voire par le biais d'un seul trait vertical dont l'unique finalité est d'éviter toute confusion sur le signe. Rien de cela n'est ici reproduit.
- Est-ce pour autant une «lune » comme nous l'avions un moment proposé (Boujot et al., 1998) en accord avec le « carré Terre » et « l'arc Ciel » la surmontant, logiquement, au dos de la stèle de chevet à la Table des Marchands ?

- Nous réfutons désormais cette proposition univoque, logique mais par trop hâtivement mentionnée dans un article qui ne s'était pas donné pour tâche principale de poser l'interprétation dans le cadre général du système des signes armoricains, car un problème de représentation se trouve posé face à la réalité de cet astre nocturne qui nous oblige, en effet, à soulever une contradiction, à souligner la confusion, à résoudre enfin l'équation.

- Habituellement reproduit par quartiers, l'astre est arqué à droite ou à gauche (de nos jours, le drapeau du Croissant-Rouge; pour la Protohistoire ouest-européenne, le signe interprété comme tel sur le fameux disque en cuivre et or de Nebra: Meller, 2002), mais ne présente que très rarement une convexité intégralement tournée vers la Terre. Tout simplement parce que la figure astronomique est durant l'année lunaire, rarement visible en l'état, aussi parallèle au «plan » de la Terre et aussi symétriquement disposée que la figure restituée ici par la gravure ; les cieux fréquemment dégagés des contextes désertiques (Sahara, Asie centrale, etc.) offrent seuls ces opportunités nocturnes pour enregistrer une telle position.

- Mais de cette impossibilité à l'observer ainsi éclairée dans le ciel, on ne peut en revanche inférer une incapacité pour l'homme à la reproduire dans cette position artificielle. On sait, dans un univers proche et actuel, la manière dont les croix des églises orthodoxes russes ou ukrainiennes surmontent le croissant basal, symétriquement disposé, ouvert sur le haut (fig. 10), transpercé (puisque symbole païen) par la croix chrétienne ${ }^{23}$.

- Mieux assuré, le dieu anthropomorphe égyptien (Iâh) revient aussitôt en mémoire, avec le disque et le croissant lunaires ajoutés sur la tête, en accord avec la position discutée ; un dieu qui sera absorbé par Khonsu, aussi bien pour son aspect agressif $($ croissant $=$ couteau $)$ que pour son

23. Ces représentations de la croix au «croissant de lune » basal, que l'on associe souvent à la victoire du christianisme sur l'islam (commémoration de la victoire du tsar Ivan IV dit le Terrible) sur la ville de Kazan en 1486), seraient, semble-t-il, connues bien avant les affrontements entre chrétiens et musulmans; pour les uns, elle signifie l'alliance de la croix et de l'ancre (Hébreux, $6: 19-20)$, pour d'autres la coupe de l'Eucharistie, et pour d'autres enfin la lune ne symboliserait que la Mère de Dieu. On ne fait pourtant pas mention dans le système de croyance orthodoxe du fait que l'Église du monde slave est très souvent pensée comme un navire. On soulignera aussi l'étrange évolution métonymique d'un signe qui, de croix passa au marteau, et de lune s'acheva en faucille au sommet des édifices publics... 


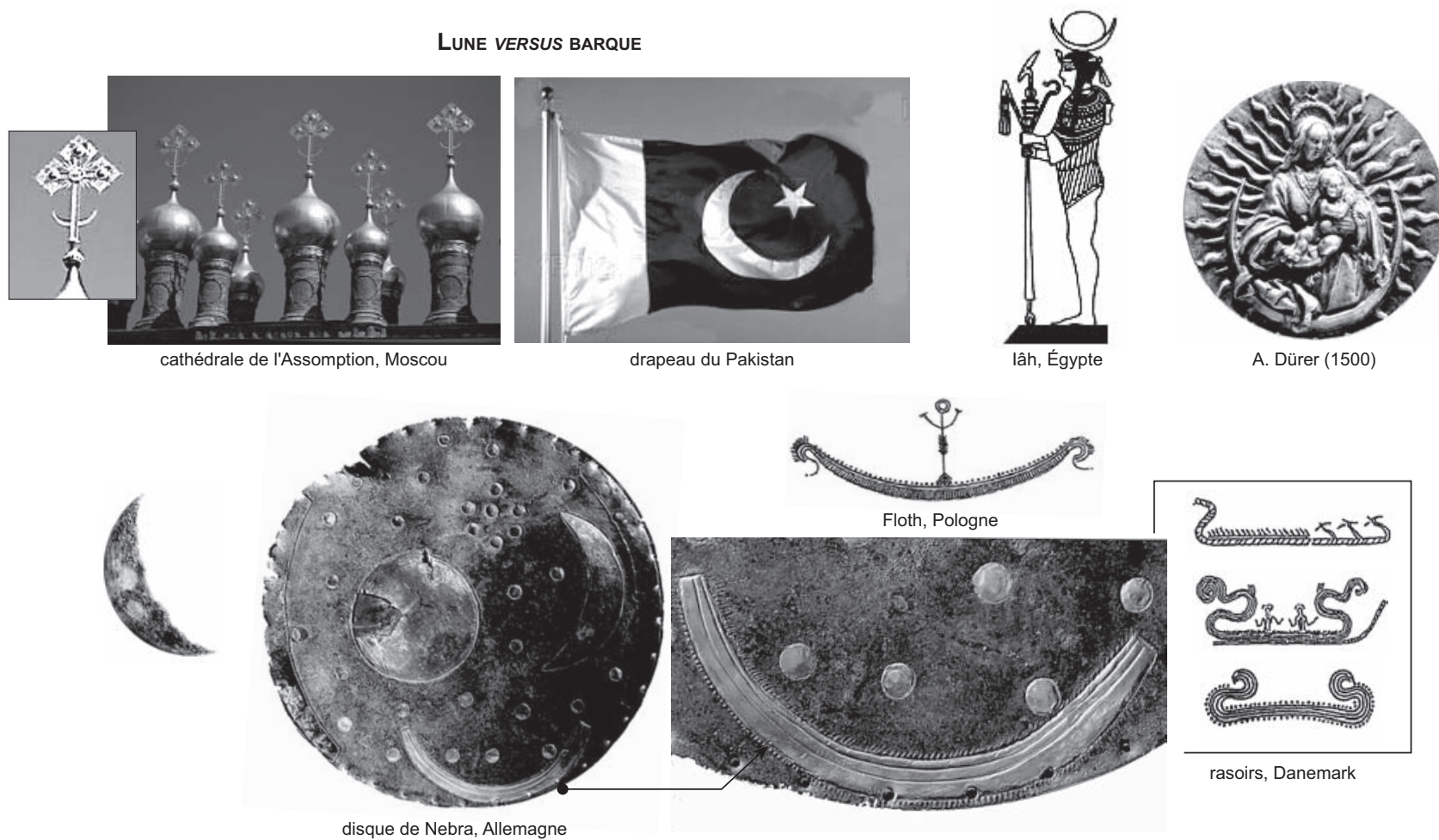

Fig. 10 - Représentations de la lune et de l'embarcation : cathédrale de l’Assomption, Moscou (cliché : S. Cassen, CNRS) ; drapeau du Pakistan (étoile et croissant lunaire) ; iconographie de Iâh, dieu anthropomorphe avec disque et croissant lunaires sur la tête, Égypte (d'après SchumannAntelme, Rossini, 2003) ; médaille attribuée à A. Dürer, 1500-1550 (d’après Knappe, 1984); disque en cuivre et or de Nebra, Allemagne : détail sur la barque tiretée et comparaisons avec les gravures de bateaux sur bronze de Floth, Pologne, et des rasoirs du Danemark (d'après Meller, 2002).

aspect de barque céleste, «le Voyageur », cette dernière qualité permettant à Iâh-Khonsu d'être le guide des étoiles, lesquelles sont aussi les âmes des défunts. Par conséquent, le dieu devint un psychopompe céleste, déjà mentionné dans les textes des pyramides (Schott, 1953 ; Tobin, 1989) ; lune et barque laissent déjà entrevoir leur perméabilité.

- C'est également par un croissant, non pas perpendiculaire mais parallèle au sol, ouvert en bas ou vers le haut et solidaire d'un motif quadrangulaire, que les hiéroglyphes hittites pourront signifier un astre (Bittel, 1976; Calvet, 1996) ; non pas la lune comme on pourrait s'y attendre mais bel et bien le soleil émergeant de la terre, la lune étant en réalité composée d'un signe complexe sans rapport avec un croissantiforme. Mais Kushukh, le dieu-lune hourrite, apparenté au hittite Arman et au mésopotamien Utu/ Shamash, porte bien le casque avec un croissant aux pointes portées vers le haut. Pour leur part, les sceaux mésopotamiens rapportent avec régularité les principaux astres, étoiles, soleil et la lune en son croissant courbe parallèle au sol (Huot, 2004).
- Autre exemple, «l'évidence» selon laquelle la forme en croissant serait une représentation en tant que telle de la lune, est largement reprise dans l'interprétation des fameuses stèles puniques de Carthage et de Numidie (Bertrandy, Sznycer, 1987), bien que le motif soit cette fois le plus souvent inversé, les «cornes » tournées vers le bas. Et alors que les symboles marins se répètent (ancres, gouvernails, poissons, dauphins) et que la représentation de l'océan supérieur censé être franchi par les âmes des défunts prend la forme de motifs ondulés (Picard, 1976), aucune hypothèse ne semble s'être portée sur la symbolisation d'un navire qui, dans ce cas, pourrait se présenter en symétrie-miroir dans un monde renversé...

- La confusion ne semble cependant plus de mise quand aux débuts du $\mathrm{XVI}^{\mathrm{e}}$ s., la doctrine de l'Immaculée Conception illustre la Vierge Marie s'inscrivant ou surmontant un croissant lunaire orienté selon les modèles antiques et proche-orientaux (voir les gravures de Dürer, fig. 10).

- Plus généralement, lune et barque échangent leur similitudes formelles, et nos discours actuels n'échappent pas 
davantage à la fusion des images (commentaires extraits de la revue Sciences et Avenir, de novembre 2005, p. 98, relatifs à des suggestions d'observations du ciel : « Le mince croissant de la vieille Lune flotte non loin de la planète Jupiter, comme une frêle embarcation [...]»).

Mais revenons au Mané Lud, et, en nous interrogeant sur la cohérence de la situation, testons l'hypothèse selon laquelle cet astre serait bel et bien le modèle de l'objet figuré.

- Que faire, tout d'abord, des personnages considérés comme tels dans notre démonstration? La forme de Kermaillard, «vide » des humains verticaux, est pourtant la même qu'au Mané Lud, bien qu'elle soit ici « pleine ». On ne peut accepter un astre ici, alors qu'il serait un bateau monté là-bas ; il s'agit d'une même ligne directrice pour signifier une embarcation, et aucune lune ne pourrait à ce point se voir déformée, aplatie. Pas de Pierrots lunaires.

- Que faire, ensuite, du contexte des signes? À la Table des Marchands, cette lune serait donc placée sous une représentation du monde? Symbole chtonien, certes, comme nous l'avancions, mais que devient ce même signe disposé cette fois le plus souvent aux côtés du «carré » ? Un astre dont on pourrait en effet admettre qu'il soit disposé «au-dessous» (chtonien) ou «au-dessus» (astronomique, mais alors dans une position aucunement inventoriée dans le corpus breton), mais qu'il n'est peut-être pas aussi conséquent d'établir suspendu "par le côté du monde ».

- Que faire, enfin, des associations de signes? Au Mané Rutual à Locmariaquer (mais aussi à Kercado à Carnac), un des deux cachalots gravés sur les orthostates en remploi dans la tombe présente son extrémité proximale au contact d'un croissantiforme. Non pas une sorte d'attribut fonctionnel confus attaché à la pseudo-hache («virole» et autre «bouterolle de protection" recensées dans les écrits des années 1980-1990), mais un objet-signe détaché de l'animal entièrement surmonté par lui et entièrement rempli par la tête quadrangulaire du monstre (fig. 11).

Quel pourrait être, en mer, cet objet sous une telle influence, sinon l'embarcation fragile sautée par la plus dangereuse des «baleines »? Quelles sont, dans l'histoire de la navigation, de l'exploration et de la pêche, les relations d'attaques, de destructions, de charpentes broyées sous l'effet de la mandibule affreuse, d'effroyables avalements, réels et embellis, souvent imaginés, puis inventés, sinon ces confrontations de canots avec la baleine à dents, la plus monstrueuse, Moby Dick, le cachalot-Léviathan ? Du Japon à l'Atlantique-sud, nombreux sont les exemples historiques d'animaux blessés ou de femelles protégeant leur petit, défonçant la paroi de bois et coulant la barque ou le navire (Lacroix, 1938).

Ces croissantiformes gravés sont des bateaux : des corps d'embarcations non montés pour certains, embarquant leur équipage pour les autres. Et justement, à ce point avancé de l'analyse, peut-on laisser une telle distinction en suspens si l'on tient à éclaircir l'image originelle ? Il faut, en effet, et dès maintenant, penser les deux termes de l'alternative. Penser en priorité la forme vide, en ceci qu'elle se différencie de la coque à personnages verticaux. Une coque vide, pur symbole... Vide ou, peut-être, renfermant la personne allongée qui fait «corps" avec l'embarcation, le corps «gisant» pour un dernier voyage, vers une terre de repos pleine de félicité, en une sorte de dormition, ou encore la première aventure vers une place fondatrice propre à toute renaissance.

Ici, une passerelle s'impose.

La traversée merveilleuse est l'une des plus grandes images inscrite dans l'imaginaire de l'Europe atlantique. Aussi nous faut-il conserver un regard à la fois attentif et bienveillant afin de procéder à la recherche de critères adéquats qui puissent nous permettre une distinction pertinente entre ces deux classes de navires. Car parmi toutes les images étranges que la littérature française médiévale doit à la "matière de Bretagne ", il en est une qui fascine : c'est celle du bateau sans voiles, sans rames, sans équipage, dérivant sur l'océan au gré des vents et des courants, conduisant de façon mystérieuse le héros vers son destin (Milin, 1989, p. 125).

De fait, l'exposition sur mer dans un bateau sans agrès constitue une ancienne pratique juridique qui allie la sanction du bannissement à la volonté de s'en remettre à une volonté supérieure à celle des hommes (id., p. 9). Le saint, lui aussi, qui accepte ou choisit de naviguer à l'aventure sur un bateau sans voile ni rame s'en remet à la volonté de Dieu, mettant justement à l'épreuve son caractère d'élu, sa sainteté et sa foi dans le Christ. C'est aussi le corps de saint Jacques le Majeur reposant étendu dans une barque, glissant vers le rivage, contre vents et marées, sans voiles, sans équipage, sans gouvernail, disait la légende. De là, cet échouage en la ria désignée, sa découverte admirative par les hommes, et de là, cette remontée du cours des eaux fluviales jusqu'à la place élective, ce Compostelle, lieu de passage qui devint le haut lieu par excellence du pèlerinage en Europe occidentale. C'est également le corps mort de saint Vincent allongé, seul, incorruptible, dans ce bateau survolé de deux corbeaux arrivant sur Lisbonne. 

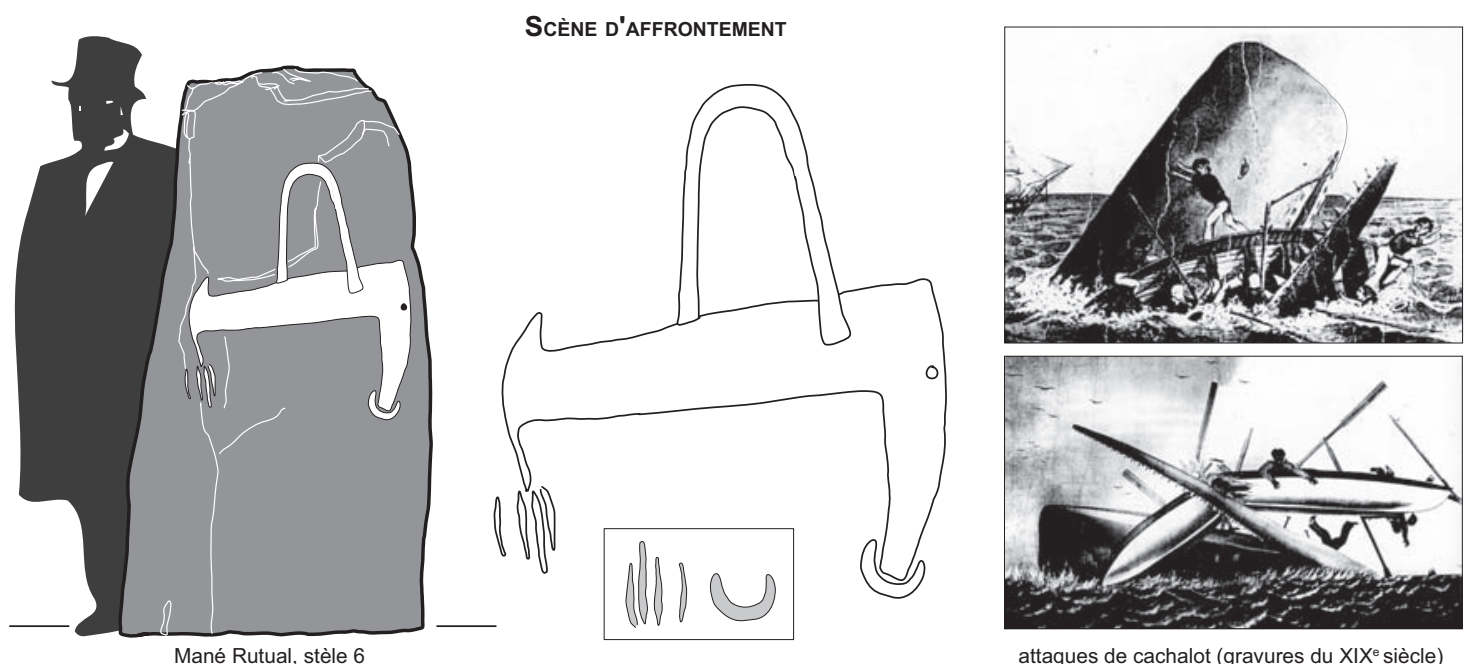

attaques de cachalot (gravures du XIXe siècle)

Fig. 11 - Représentation gravée sur la stèle 6 du Mané Rutual à Locmariaquer, Mobihan (Cassen et al., 2005), association des traits verticaux sur la queue et de la forme en croissant à l'extrémité de la tête du cachalot : gravures du XIXe s., attaques de cachalot sur des baleinières (d'après Lacroix, 1938).

Enfin, le bateau de pierre transportant le saint, dans toutes les chrétientés celtiques, passe pour avoir une vertu guérisseuse. Voilà pourquoi, si vous remontez cette ria coulant au pied du Mané Lud, si vous flottez avec la marée dynamique dans le cours de la rivière salée (le Sal), vous arriverez au bas de la chapelle de Sainte-Avoye à Pluneret (Morbihan) où un bloc de pierre est le bateau que la sainte prit pour venir de Cambrie en la rivière d'Auray (Milin, 1989, p. 136).

Dans ces exemples, on le sait, les récits de traversées miraculeuses viendraient christianiser les formes occidentales connues d'un certain « culte des pierres » impossible à éradiquer ; ainsi le pouvoir miraculeux du bateau de pierre (pierre guérisseuse, pierre de fécondité, pierre de serment), trouverait une explication dans le miracle initial que constitue la traversée du saint sur cette embarcation paradoxale : l'hagiographie christianiserait ainsi des pratiques bien antérieures au culte des saints guérisseurs (Milin, 1991, p. 19). Mais des pratiques qu'il serait peut-être aussi simple de rapprocher de ce «miracle » initial, des pratiques à nos yeux indissociables du phénomène fondateur, et, lui, bien réel et passé dans la mémoire incommensurable des hommes: autrement dit, ces dizaines de stèles en orthogneiss, au poids inimaginable (300 tonnes au moins pour le Grand Menhir) qui ont parcouru des kilomètres et traversé l'étendue d'eau et remonté les pentes avant d'être fixées en leur lieu d'élection (Le Roux, 1997 ; Cassen, 2004 et 2005).

On voit donc se dessiner un bateau, réserve d'imaginaire, figure mythologique, une figure donnant par essence cette pleine force narrative, d'une importance comparable aux grandes entités jusqu'ici découvertes : cachalot, pigeoncorneille, animaux cornus, crosse de jet, hache tranchante... Du reste, une entité aussi fondamentale serait-elle à ce point isolée et sa formule restreinte à quelques stèles morbihannaises? Assurément non, et de cette identification dans le registre des gravures armoricaines découlent, comme il semble probable, quelques conséquences secondaires dans le temps et l'espace ouest-européen, au cœur du processus général de reconnaissance des formes simples qui, par rétroaction, donneront alors crédit à notre démonstration.

- À l'échelle régionale : une forme comparable est identifiée dans le corpus céramique breton (fig. 12). Cette forme modelée, "cornue» encore une fois, fut même posée, signifiante en soi, pour définir un style céramique, le Carn (L'Helgouac'h, 1965), sous-ensemble «funéraire » de l'entité plus élargie que nous avons nommée AuzaySandun (Boujot, Cassen, 1992 ; Letterlé, 1992 ; Cassen, 1993 et 2003). La question est donc posée car l'identité est manifeste.

- À la dimension de la façade atlantique de l'Europe et dans un créneau historique comparable : car la réplication de l'assemblage «carré-croissant ", bien visible sur les stèles contemporaines du Portugal méridional à Portela de Mogos (Varela Gomes, 1997a) (fig. 13), ne peut échapper à l'observateur attentif (Briard, 1995, p. 184) ; mais une fois cette coïncidence identifiée, elle ne doit pas en rester au stade de la simple convergence de forme entre deux aires géographiques éloignées, ou encore nous faire à nouveau tomber dans ces 


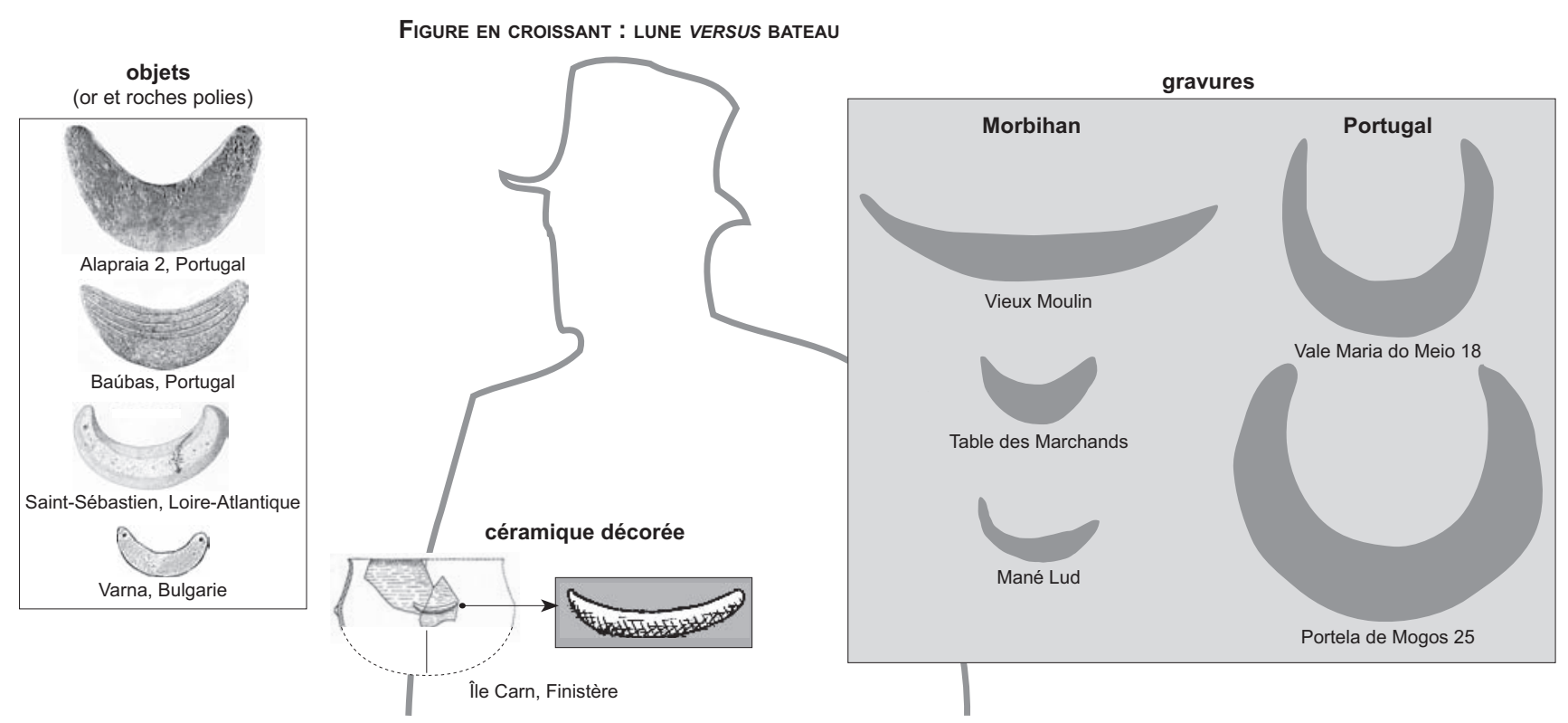

Fig. 12 - Objets et gravures croissantiformes comparés : croissants en calcaire des tombes "chalcolithiques » de Baúbas (Carenque) et Alapraia 2, Portugal (d'après Leisner, Leisner, 1965) ; croissant en dolérite polie de Saint-Sébastien-sur-Loire, collection Pitre de Lisle, musée Dobrée, Loire-Atlantique (d'après Gouraud, 2003) ; croissant en or, sépulture $n^{\circ} 4$ de Varna, Bulgarie (d'après Ivanov, 1989) ; céramique décorée de l'île de Carn, Finistère ; gravures des stèles de Vale Maria do Meio 18 et Portela de Mogos 25, Portugal (d'après Varela Gomes, 1997a et b) ; gravures des stèles dans la tombe à couloir du Mané Lud et de la Table des Marchands à Locmariaquer.

surdéterminations astronomiques mal fondées quand le signe croissantiforme est compris comme l'astre nocturne (Calado, 1997, p. 296 ; Varela Gomes, à paraître). Mieux encore, la crosse ajoute par sa présence singulière un appui supplémentaire à une armature «syntaxique» commune entre des régions bien éloignées. Une liaison de représentation également établie avec cette zone intermédiaire du Portugal septentrional et de la Galice, où saute et souffle une même abstraction cachalotesque (Cassen, Vaquero, 2000).

- À l'appui de la longue durée : car le disque " céleste » de cuivre et d'or découvert à Nebra-sur-Unstrut, SaxeAnhalt (Meller, 2002), daté du Bronze ancien, réunit deux formes-forces en présence déjà contenues au Mané Lud, un millénaire plus tôt, et sur lesquelles il nous a fallu discuter : la lune et la barque (fig. 10). Ici en Allemagne, la lune est lune parce que son croissant correctement orienté est opposé à un disque, évidemment "solaire" (bien que ce dernier puisse aussi renvoyer à une pleine lune) et dans un ciel étoilé dominé par de possibles Pléiades; l'autre croissant est barque parce que bordé de tirets et démuni aux extrémités de pointes comparables au croissant précédent, et peu importe pour notre propos qu'il soit embarcation solaire ou bateau des morts. L'enquête de H. Meller est d'ailleurs convaincante pour nous aider à reconnaître un bateau, rapprochant avec profit toute la longue tradition des représentations de navires dans le monde septentrional européen, sur différents supports, depuis les affleurements rocheux jusqu'aux rasoirs en bronze, sans oublier la boucle de ceinture de Floth en Pologne (IX ${ }^{\mathrm{e}}-\mathrm{VIII}{ }^{\mathrm{e}}$ s. av. J.-C.) où, dans chacun de ces exemples, rames et personnages sont finalement réduits à de simples tiretés, parfois identiques en taille et en position aux incisions portées de part et d'autre du croissant de Nebra.

- Enfin, en testant l'idée, la logique interne de reconnaissance et la pertinence de nos interprétations sur un lieu distinct et comparable tout à la fois. À l'image de la nécropole hypogée d'Anghelu Ruju à Alghero, ou encore de celle de Montessu à Villaperuccio en Sardaigne (Tanda, 1979) où les sépulcres hypogées du III millénaire, de type Domus de Janas, laissent entrevoir des " cornes" et des têtes de taureau dégagées en bas-relief (fig. 14). Des cornes taurines, donc, mais en forme de «barque" « a barca» (Moravetti, Tozzi, 1995, p. 45), car, en effet, l'analogie semble couler de source. Une analogie finalement bienvenue, n'est-ce pas, pour accompagner des populations et des sites au contact de la mer qui ceinture. 


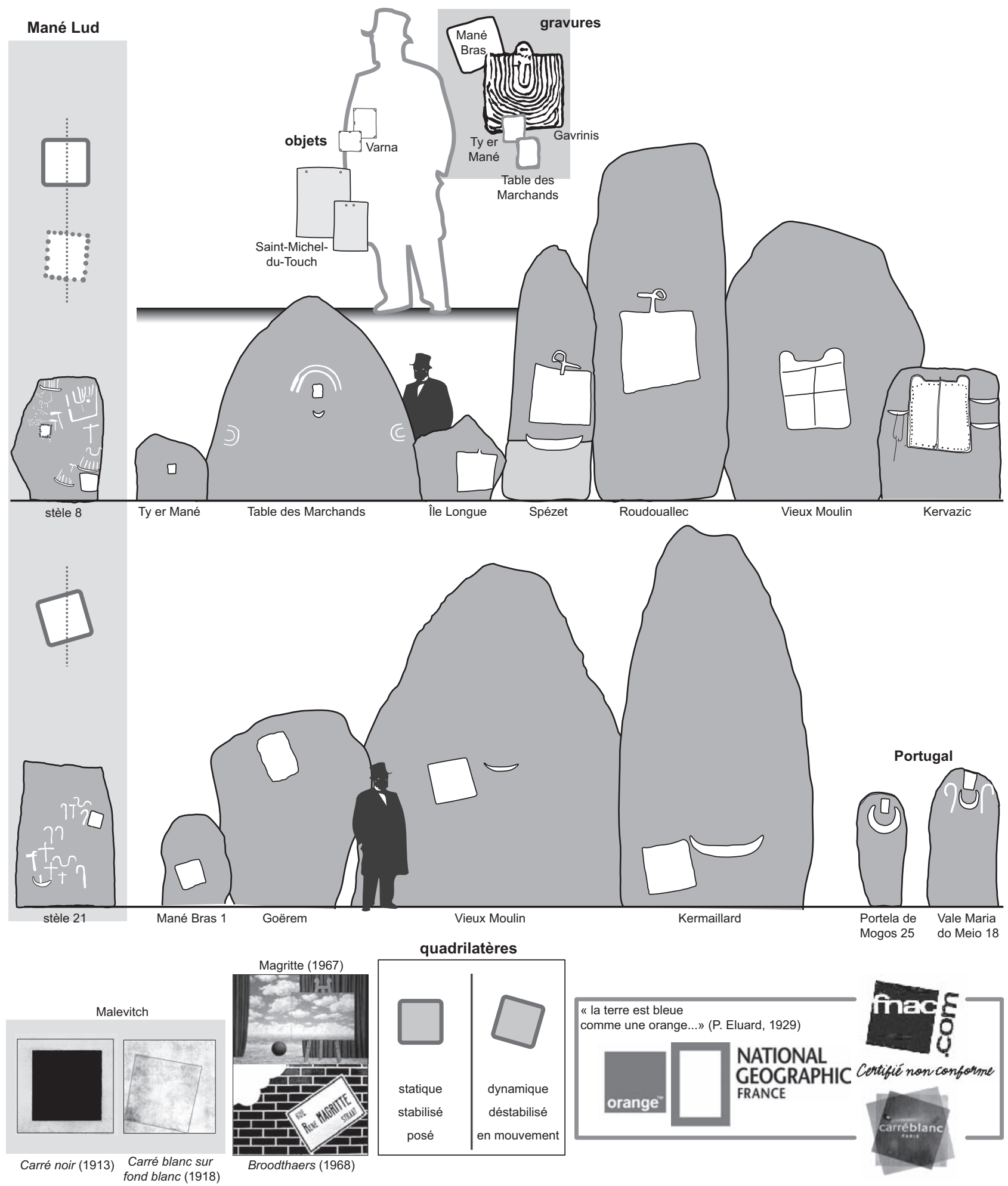

Fig. 13 - Le motif quadrangulaire, objet et gravure, une opposition de placement dans la représentation : stèles encore dressées ou en réemploi de Bretagne et du Portugal; Portela de Mogos 25 (d'après Varela Gomes, 1997a) et Vale Maria do Meio 18 (d'après Calado, 2004) ; mise en correspondance des gravures quadrangulaires et des plaques en pierre à Saint-Michel-du-Touch, Haute-Garonne (d'après Méroc, Simmonet, 1979) et en or, sépulture $n^{\circ} 4$ de Varna, Bulgarie (d'après Ivanov, 1989) ; peintures de Malevitch (http://www.kandaki.com/CM-Art.htm), de Magritte et de Broodthaers (d'après Diserens, Chevrier, 2003) et logos quadrangulaires droits et penchés d'entreprises commerciales. 


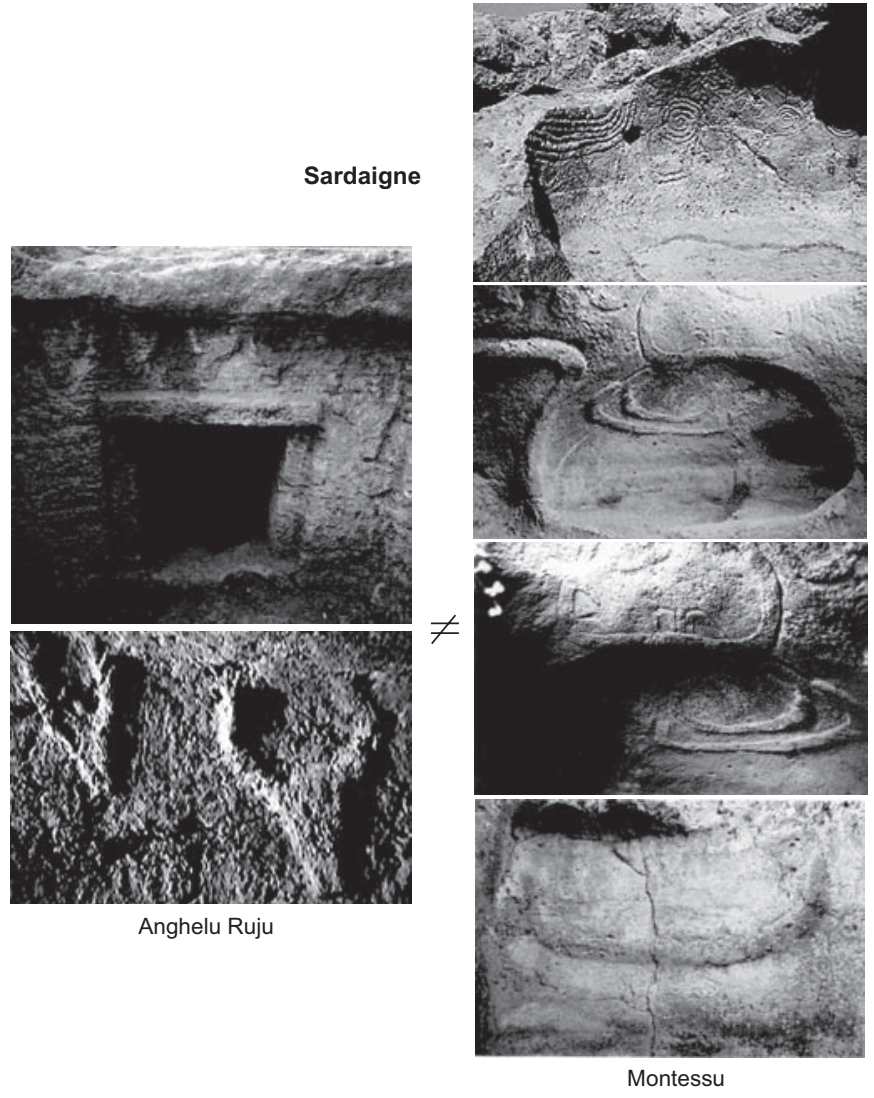

Cornus versus bateau L'EXEMPLE SARDE
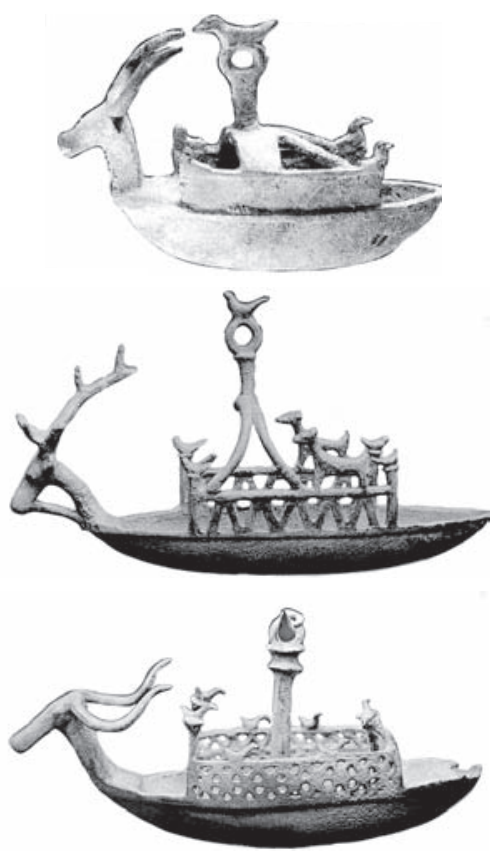

navires (bronze) nuragiques

Fig. 14 - Distinction entre les représentations de bucranes vrais et autres "cornus » des hypogées sardes : gravures en champlevé de la nécropole hypogée d'Anghelu Ruju, Sardaigne (d'après Moravetti, Tozzi, 1995) ; gravures de la nécropole à Domus de Janas de Montessu, Sardaigne (d'après Moravetti, Tozzi, 1995 ; http://www.stonepages.com/sardinia/sites/montessu_spirali.html) ; navires nuragiques en bronze, entre 1200 et 600 av. J.-C. (d'après Guerrero Ayuso, 2004).

Aussi nous faut-il être cohérent et sincère avec les images : pourquoi juxtaposer en ces tombes, d'un côté, de vrais bucranes, tête apparente, tête réaliste ou simplifiée sous figure quadrangulaire mais supportant logiquement et dans tous les cas les encornures, et d'un autre côté ces «cornes» isolées qu'aucun frontal ne vient souder? Et pourquoi ne pas admettre que ces «cornes", symétriques et parfois singulièrement et inexplicablement asymétriques, n'évoquent plus du tout des encornures dans leur diversité ? Qu'en somme l'image spontanée (et refoulée) donnée par nos collègues est bel et bien celle qui convient: voici peut-être, en Sardaigne, des représentations de navires, d'autres navires en contexte funéraire. Des bateaux que les maquettes et miniatures funéraires confectionnées dans le bronze, plusieurs siècles après, révèleront guidés en proue par des têtes de cerfs et de taureaux, tandis que des «pigeons» surmontent le pont sans équipage (Guerrero Ayuso, 2004)... Comme un déjà-vu...
Des cas d'espèces similaires confondant les formes, ici par irréflexion sur l'idée préconçue, là par une croyance convenue en la polysémie fatale du signe archéologique. Des exemples semblables à celui menant, par un processus identique, à la confusion décrite au long de l'historiographie du Mané Lud.

Lune, cornes et barque échangent leurs protubérances, leurs ressemblances formelles, leurs histoires et leurs étymo$\operatorname{logies}{ }^{24}$.

24. II est un fait que l'association de la lune et du taureau est bien connue des Sumériens et aussi des Hindous, tandis qu'au Cambodge on désigne la lune comme une corne parfaite; Corniculans luna est pour les Romains la nouvelle lune, la lune en son croissant (Freund, 1840). G. Durand (1969, p. 285) aimerait aussi montrer que les cornes des bovidés sont le symbole direct des « cornes » du croissant de lune, morphologie sémantique qui se renforcerait par son isomorphisme avec la faux ou la faucille du Temps, instrument de mutilation, symbole de la mutilation de la lune qu'est le croissant, le "quartier " de lune. 


\section{L'ÉCUSSON}

Il s'agit de la figure ultime de notre liste, placée au terme de notre analyse car son interprétation s'appuie en partie sur la résolution des signes précédemment ordonnés. Une figure, enfin, résultat historique d'une autre confusion de formes.

\section{DÉCOMPOSITION}

Voici la figure « en écusson », la « déesse à l'écusson », la divinité néolithique, féminine ou asexuée, et au Mané Lud, avec plus de difficulté dans les écrits du $\mathrm{XX}^{\mathrm{e}} \mathrm{s}$. étant donné les réticences à s'accorder sur la ligne graphique qui ne fait pas l'unanimité, la «Déesse-Mère ». Et c'est le vicomte de Pulligny qui restera probablement celui par qui le nom sera propagé (1879, p. 219), bien que son ouvrage tombe aussitôt dans l'oubli des référentiels bibliographiques; on répètera son emploi « héraldique » jusqu'à nos jours, par habitude des mots-outils en usage et par une association d'idées qui ne choque ni la vue ni l'esprit.

Mais parce que les premiers relevés de L. Davy de Cussé, en 1865, ont déformé la figure quadrangulaire gravée au bas de la stèle $n^{\circ} 6$, l'affublant d'un pointement qui n'existe pas, et que cet ajout artificiel, déterminé par un accident à la surface de la pierre (Cassen et al., 2005), a dicté le sens des interprétations et la qualité même des relevés postérieurs qui ne remettront plus jamais en cause ce détail puisqu'il est attesté sur des gravures en apparence si semblables, « l'écu » reconnu par le vicomte de Pulligny est dès lors comparé aux cartouches égyptiens ou, mieux, à la gravure similaire de Boury-en-Vexin, et plus généralement, bien sûr, aux figures majeures du Mané er Hroëck et de la Table des Marchands 25.

25. À ce propos, on rappellera l'interprétation donnée par l'auteur, favorisant des «habitations" pour expliquer les figures de ces deux derniers sites, qui en raison justement de l'écartement notable de leur ligne graphique par rapport au modèle mis en cause au Mané Lud, permet ce rapprochement avec le domaine géographique procheoriental, souverain à l'époque: "Sur le dolmen du Mané Lud, en Morbihan, l'on trouve, il est vrai, un écu qui diffère peu des cartouches des hypogées égyptiens ; mais cet écu, qui aurait beaucoup de rapport avec celui de Boury, est renversé la pointe en haut, ce qui lui fait perdre tout son caractère. Il en est de même de ceux du Mané Er'Hrock, et de la Table de César ou des Marchands (Daul-Varchaut). Toutefois ce dernier, à la forme cylindro-conique, ressemble plus à ces tentes des tribus asiatiques enlacées de feuillages sur lesquelles on jetait un tissu, premier type du dôme si commun en Orient. Il existe encore en Asie Mineure quantité de ces tentes faites de terre, façonnées à la manière des ruches. »(Pulligny, 1879, p. 219) ; ces types d'habitations procheorientales, mêlant les végétaux à leur construction, ont été récemment repris par un collègue russe, de façon tout à fait indépendante, pour à son tour interpréter notre « déesse » (Smirnov, 2001).
Le motif quadrangulaire sur la stèle $n^{\circ} 6$ appartient-il en définitive à la même famille canonique de tracés que celle habituellement désignée par l'expression «Déesse en écusson »?

- On rappellera, tout d'abord, que cette dernière figure emblématique - dont le modèle de base est le mieux décliné, parmi d'autres sites, à l'Île Longue, à Mané Rutual ou à la Table des Marchands -, pourrait être désormais assimilée à un phallus (Cassen, 2000c), suivant un dispositif de reconnaissance graphique et archéologique difficile à résumer ici en quelques courtes lignes. En tout cas, la réfutation de ce travail et une contestation portée sur le sujet devront au préalable défaire notre construction; puis convaincre, par un autre biais que la seule pétition de principe servant le plus souvent d'argument pour stipuler tour à tour une déesse, une idole féminine, une divinité anthropomorphe, un bouclier, une habitation et une meule de foin.

- On insistera, ensuite, sur l'absence flagrante de ressemblance, même minime, entre le quadrilatère du Mané Lud et les figures précitées : aucune courbe chez le premier, aucun détail significatif appartenant au motif phallique (demi-cercles latéraux, pointement, etc.) chez les secondes.

- On concédera cependant qu'il peut subsister, en certains cas, une discussion ouverte sur le sujet. À l'île Longue, par exemple, une figure quadrangulaire semblable en cela au Mané Lud est néanmoins différenciée par une interruption de sa limite prenant la forme d'une excroissance sur le côté «supérieur» du carré ; un détail qui fut généralement interprété comme une simplification extrême de la «déesse » (le «pointement apical » ou la «tête»), contemporaine de la fin du Néolithique. Seulement, la discussion naît davantage, à nos yeux, de son face à face physiquement réalisé avec l'autre stèle du couloir où le motif phallique est clairement reconnu sans ambiguïé, avec toutes ses unités constitutives. Comment donc concevoir, en un même lieu, ce décalage historique dans les représentations, sinon par deux projets sémiologiquement distincts?

Autre exemple, au Berceau à Saint-Piat (Eure-et-Loir), en plein Bassin parisien, la forme carrée de «l'idole » réunit cette fois certains caractères qui semblent en effet signifier le phallus morbihannais («cheveux» ou traits divergents, « oreilles» ou demi-cercles latéraux). Dans la mesure où une forme aussi radicale serait en usage au Berceau, pourrait-on conclure que le carré du Mané Lud serait cette abstraction poussée d'un phallus? Répondre à une telle interrogation imposerait de longs développements, sans prise directe avec les objectifs de cet article. Soulignons seulement l'existence d'un processus général d'abstraction à l'œuvre pour un 
contexte géographique éloigné des côtes armoricaines, qui pourra d'ailleurs s'appliquer aux représentations encore plus lointaines, sur les stèles bourguignonnes où la question de l'identité du motif se pose en termes voisins.

Plus généralement, ce sont tous les motifs quadrangulaires, simples ou enrichis de détails sommitaux (« haches »), attribués au Néolithique final breton, qui mériteront d'être adjoints au corpus discuté. Dans l'immédiat, posons que le signe quadrangulaire simple du Mané Lud est étranger à la complexe figuration phallique, et qu'il s'agit bien d'un autre motif du corpus armoricain dont l'interprétation reste à vérifier, sinon à établir.

\section{COMPOSITION}

Le motif quadrangulaire (carré, rectangle, trapèze) est repris sur les stèles $\mathrm{n}^{\text {os }} 2,6,16,19$ et $21^{26}$. Deux traitements techniques différencient ces figures, selon que leur tracé est dépendant d'une gravure continue, ou au contraire redevable d'une gravure discontinue générée par une suite de ponctuations ou cupules $\left(n^{\circ} 6\right)$. Cette opposition sera un des éléments fondamentaux structurant notre interprétation finale (Le Mané Lud en mouvement), mais contentons-nous pour l'instant d'en marquer la dichotomie, en constatant cependant l'exacte reconduction de l'idée et la reproduction du procédé dans le registre décoratif céramique du Castellic (la ligne cannelée s'opposant à la ligne ponctuée), pour ne prendre ici qu'une seule référence régionale convergente.

Comment se répartit le motif au sein des assemblages de signes? :

- il est entouré de bateaux montés (stèle $n^{\circ} 6$ ), sur le côté et sur le dessus ;

- il est surmonté d'un oiseau (stèles $\mathrm{n}^{\text {os }} 6,16,19$ et 21) ;

- il est strictement associé à une crosse sur son côté (stèle $\mathrm{n}^{\mathrm{o}} 21$ ), voire deux (stèle $\mathrm{n}^{\mathrm{o}} 2$ ?) ;

- il est surmonté de haches (stèle $n^{\circ} 6$ ).

Comment l'orientation du motif pourrait-elle jouer sur le placement des autres signes? :

- son basculement à droite détermine une orientation conforme de la crosse la plus proche (fig. 1), mais n'impose

26. Notre rapport d'intervention (SRA, 2003) et l'article technique décrivant nos résultats (Cassen et al., 2005) laissent un contour incomplet au motif quadrangulaire en haut de la stèle $\mathrm{n}^{\circ} 21$, faute de pouvoir accéder à la figure alors enfouie sous des sédiments; un sondage restreint mené en mai 2005 a permis cette fois de fermer et d'affirmer le périmètre complet, et d'assurer en fait le dessin d'un carré. pas cette position à la première hache associée sur le panneau (stèle $\mathrm{n}^{\mathrm{o}} 21$ ) ;

- de même, la base du motif placée en parallèle au sol ne détermine aucunement une orientation similaire de la hache (stèle $n^{\circ} 6$ ) qui est, en réalité, penchée vers l'arrière.

Ce jeu des orientations, qu'il serait vain de quantifier en nombre de combinaisons ou de figer en termes de présence/absence, est peu interprétable si nous le déconnectons du dispositif général de la scène, du regroupement des stèles et des situations récurrentes dans le registre armoricain ; aussi, renverrons-nous le lecteur à l'interprétation finale des scènes enregistrées au Mané Lud, à la lumière d'une éventuelle syntaxe lisible sur le site pris dans son intégralité. Mais nous pouvons, en attendant la parution de cette étude, et sans les interpréter, compléter l'inventaire en rappelant et précisant quelques associations de signes observées en Bretagne qui fonctionnent manifestement bien avec ce motif quadrangulaire.

\section{COMPOSITIONS ARMORICAINES}

On distinguera deux critères de différenciation et d'analyse, ainsi que l'impose dans ces lignes notre approche des stèles du Mané Lud: la nature des signes en contact mutuel, et les orientations des motifs. Des exemplestypes seront mentionnés pour mémoire et contrôle: site, commune.

Le motif quadrangulaire, à tracé simple, est distingué (fig. 13) :

- isolé au centre de la stèle (Ty er Mané à Erdeven, Morbihan);

- en présence d'un bateau non monté, en position latérale (Vieux Moulin à Plouharnel, Morbihan) ;

- en présence d'un bateau non monté, en position sousjacente (Table des Marchands à Locmariaquer, Morbihan) ; - en présence latérale d'un signe mal identifié (« marteau ») (Kermaillard à Arzon, Morbihan) ;

- en présence (douteuse) d'un double mamelon (Mané Bras à Erdeven, Morbihan).

Le motif quadrangulaire, à tracé complexe (ligne de contour continue ou discontinue; partition de la surface interne délimitée par le motif), est distingué :

- en présence d'un bateau non monté, en position sousjacente (Le Duc à Spézet, Finistère) ;

- en présence d'un bateau non monté, en position latérale (Kervazic à Erdeven, Morbihan) ;

- en présence latérale d'un signe mal identifié (« la Palette») (Prajou-Menhir à Trébeurden, Côtes-d'Armor) ; 
- par adjonction d'une hache en position sus-jacente (Roudouallec, Morbihan).

Par rapport à l'axe longitudinal vertical de la stèle, les orientations des motifs quadrangulaires seront, à l'image du Mané Lud :

- obliques, le signe penché à droite ou à gauche (Kermaillard, Le Duc, Mané Bras, Vieux Moulin), quand la composition est asymétrique par rapport à cet axe ;

- ou normales suivant cet axe (Table des Marchands, Ty er Mané), au sens géométrique du terme, quand la composition des signes est symétriquement disposée.

Enfin les supports renvoient le plus souvent à des stèles :

- «isolées » ou placées en files à la surface du sol ;

- en remploi dans les tombes à couloir datées de la fin du Ve et du début du IV millénaire ;

- en position primaire dans les tombes de la fin du Néolithique si l'on se réfère à la littérature sur le sujet (Giot et al., 1998).

De cette liste, sous l'impact de ces nouveaux noms, de ces situations inédites et des intrigues conséquentes, on devine qu'un champ important s'ouvre à une recherche également renouvelée qui permettrait une meilleure compréhension des agencements de signes dont l'actuel cloisonnement artificiel en familles, selon les types architecturaux des tombes hébergeantes ou suivant la dominante graphique qui les distinguerait, devra être repensé à l'échelle du IV millénaire. Un tel champ doit aussi bien participer à une plus fine résolution des problèmes déjà recensés de chronologie relative, qu'à la perception sensible de la cohérence et de la permanence du système des représentations non seulement en Armorique, mais encore dans le nord de la France.

Cela dit, revenons au Mané Lud et reprenons le motif quadrangulaire à la lumière des nouvelles propositions relatives aux autres signes; interrogeons ensuite chaque scène afin de déterminer les relations cohérentes pouvant exister entre ceux-ci et le motif, de sorte que puisse se dégager une interprétation plausible de ce dernier.

\section{RECOMPOSITION}

Voici donc un signe géométrique gravé au contact de mots, de choses, d'animaux et de caractères bien " réels », sinon bien identifiés :

- un signe systématiquement survolé par un oiseau ;

- un signe au contact, voire entouré de bateaux montés par un équipage ;
- un signe au contact direct d'une arme de jet, la crosse, objet-signe des temps anciens ${ }^{27}$;

- un signe au contact plus éloigné d'une arme inédite et terrible, la hache, objet-signe des temps nouveaux.

Quelle pourrait être une telle image? Pour commencer, peut-on soumettre à notre vue les dimensions d'un tel sujet perçu? :

- sa taille est bien grande par rapport au modèle du bateau monté qui affronte la mer ;

- sa taille est alors trop réduite par rapport à l'oiseau qui le surmonte d'aussi près ;

- sa taille est cependant comparable aux objets utilisés par les hommes (crosse, hache).

Voici en conséquence un sujet fondamentalement contradictoire dont il nous faut rendre compte, une contradiction qui ne peut se résoudre que dans la pensée et l'image de l'immense :

- quelque chose vu du ciel des oiseaux ;

- quelque chose entouré de l'océan des bateaux ;

- quelque chose qui serait alors miniaturisable à la mesure des armes de l'homme, pour devenir en conséquence objet lui-même, un «symbole " comme le seraient la crosse et la hache dont on fait des bijoux d'or et de pierre rare, et que l'on porterait en réduction.

Avant de l'envisager comme un objet matériel répondant à toutes ces contraintes, posons la question : cette forme, ne pourrait-elle pas figurer un animal, «énorme » et «sur l'eau », suivant en cela la logique de notre suite descriptive?

La nature n'en porte aucun dessiné selon ces lignes, variant du carré au rectangle, bien que l'abstraction d'un animal soit, bien entendu, plausible. Et l'on pense dès lors au cachalot du bestiaire des finisterres européens dont la tête quadrangulaire (Gavrinis, Grand Menhir), sur laquelle le naturaliste Lacépède insistait en son temps pour l'assimiler à un carré (Cassen, Vaquero, 2000, p. 635), se prêterait aisément au processus de simplification et de symbolisation.

Mais on perçoit une certaine difficulté à concevoir qu'un bateau soit figuré de manière aussi réaliste tandis que l'animal placé à son contact serait limité à sa seule tête géométrique, sans jet, sans queue, une géométrie de tracés qui, nous le savons, n'autorise la reconnaissance qu'en rapport avec d'autres unités explicites (queue, bosse, pénis, etc.). Seule la configuration du motif de l'Île Longue pourrait trahir le signifié, par l'intermédiaire de cette interruption

27. Pour une discussion sur l'antériorité de la crosse des chasseurs sur la hache des agriculteurs, et sur la préséance de cette dernière sur la première, voir Cassen, 2000e et 2007 ainsi que Cassen, Vaquero, 2003. 
et de cette excroissance dans le tracé, relevées sur le côté supérieur, qui seraient - pourquoi pas ? - assimilables à l'évent et/ou au souffle de la bête.

Il n'en reste pas moins qu'une scène de chasse ou de rencontre entre l'homme et l'animal - des complexes iconographiques dont les composantes respectives seront restituées en proportions et en niveaux d'abstraction comparables pour pouvoir fonctionner de concert- serait efficacement rendue en suivant des normes de représentation compatibles, cohérentes, a fortiori si nous savons qu'en d'autres occasions le cachalot peut être reproduit et identifiable malgré une simplification radicale.

Si la forme n'est donc pas celle d'un animal, quel objet serait à même de subir autant d'impositions selon la taille (gigantesque), le milieu (marin) et la capacité à la miniaturisation?

Certainement pas un objet-outil dont nous aurions déjà trouvé trace.

Afin d'en comprendre la nature, d'interpréter le signe en répondant à ces questions, rassemblons maintenant quelques données archéologiques éparses - appartenant au lointain passé comme au présent - sur une forme tout à la fois singulière et répandue, afin d'illustrer l'exercice comparatif (fig. 16) :

- voici la forme géométrique peinte ou gravée, parfois partagée, puis encore divisée pour donner le premier damier, une forme quadrilatère qui se trouve déjà figurée avec les animaux à Lascaux (Leroi-Gourhan, Allain, 1979 ; Breuil, 1985), comme "piégeant» parfois leurs extrémités (sabots, queue) ;

- une forme que des objets énigmatiques vont exactement reproduire, selon le carré ou le rectangle, en des matériaux précieux qui feront d'eux ces biens socialement valorisés, à l'image des appliques en or de Varna et des plaques à suspendre en grès de Saint-Michel-du-Touch, toutes en contexte funéraire et pour s'en tenir dans un premier temps à un horizon chronologique comparable à la Bretagne ;

- des contextes funéraires qui, à cette époque, permettent de produire des volumes céramiques cubiques, exceptionnels en Bretagne (Moulin des Oies à Belz, voir Cassen, 2003), plus fréquents à l'échelle des fameuses coupes-à-socle du Bassin parisien et de la région toulousaine, et seulement sur cet unique modèle (François, 2001) ; des coupes-à-socle elles-mêmes inscrites de motifs quadrangulaires divisés, de vrais damiers, brûle-parfums que nous sommes tenté de placer en équivalence exceptionnelle avec des plats supposés devoir servir à la production du sel (fig. 15) (Cassen, 2000f; Cassen et al., 2004), en sorte que deux processus physico-chimiques remarquables, la fumigation d'une part, la fusion-cristallisation d'autre part, vont tous deux déployer leur pleine fonction de "passage » à travers ces formes décorées, en assurant la transition entre deux états au sein du rapport fondamental à l'altérité, qu'il s'agisse de possibles divinités d'en haut ou d'entités bien humaines ici bas 28 ;

- des contextes qui semblent se répondre de loin en loin par la singularité des céramiques à ouvertures carrées ou quadrangulaires, ponctuellement présentes dans l'Europe des $V^{e}$ et $I^{e}$ millénaires, depuis les Balkans jusqu'à la Normandie, en passant par la Catalogne, le Puy-de-Dôme, l'Yonne ou l'Allemagne (Bazzanella, 1997), mais sans que la Scandinavie ou encore les îles britanniques, dernier cercle de la «néolithisation » ouest-européenne, ne soient affectées par l'idée, si, toutefois, communautés de conception et de croyance ont pu être partagées entre toutes ces régions souvent séparées ;

- une forme et un contour géométrique, enfin, qui transcendent le seul mobilier céramique si accessible, pour mieux s'accomplir, ici dans le dessin au sol des villages du Néolithique ancien centre-européen (fig. 16), là dans le plan des maisons d'Irlande en plein $\mathrm{IV}^{\mathrm{e}}$ millénaire (Drummenny, Coolfore, Corbally, etc., voir Purcell, 2002), ou encore transposés dans les cairns et les chambres sépulcrales d'Armorique : autrement dit des projets distincts, des complexes architecturaux éloignés mais pourtant redevables pour leur implantation d'un orient assez simple qui les dispose, dans l'espace électif, en rapport avec la conception de l'espace, avec les directions cardinales et les points fixes et attendus du cycle du temps qui passe, devient et revient.

\section{LE JARDIN EXTRAORDINAIRE}

Voici une forme dont la force évocatrice est telle, on le voit, que le plan du village, de la maison et de la tombe, suivront ensemble ce dessin.

28. La compréhension d'un tel rapport, extrait de ces formes céramiques et en vertu de leur «décor", est en réalité en partie visible au sein des productions relevant d'un même principe d'ordination et d'interprétation, depuis les damiers sur les vases à pied de la TRBK («tambours" des contextes funéraires) jusqu'à ceux marquant les drapeaux agités en fin de course automobile, en passant par les échiquiers médiévaux (un jeu d'affrontement bipartite que le graphiste retrouve en sa tragédie initiale à travers la création du logo Sidaction 2005 , voir fig. 15), bref un phénomène universel impossible à résumer dans cet article et qu'il conviendra de traiter séparément, en partant justement de l'invention de ces types céramiques si particuliers dans les commencements du Néolithique, ou encore en sollicitant les «damiers » de Lascaux... 


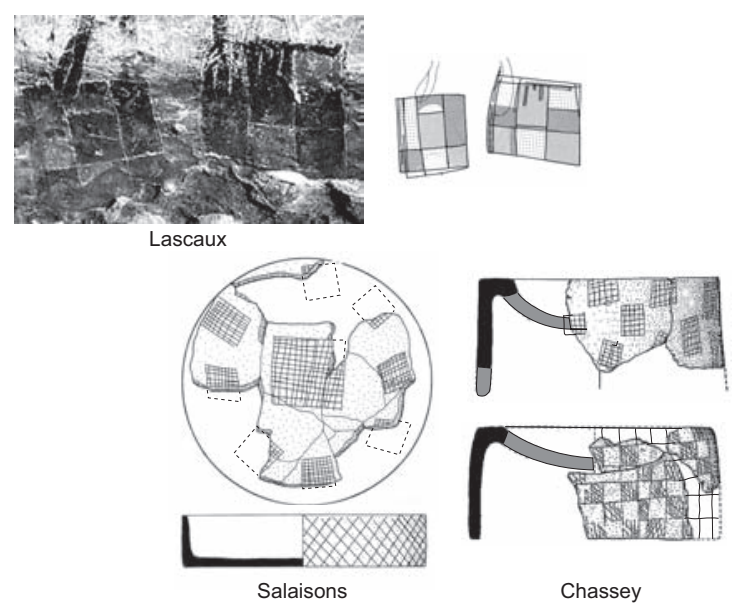

Figures de L'ALTÉRITÉ : LE QUADRILATÈre SÉPARÉ, DIVISÉ
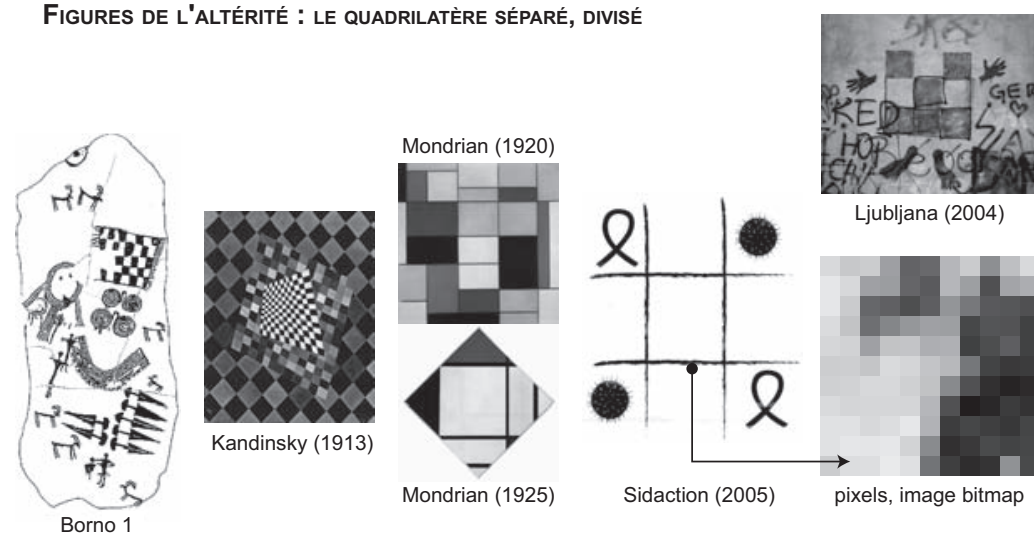

Ljubljana (2004)

Fig. 15 - Le motif quadrilatère à travers un raccourci historique de ses représentations : Lascaux, Dordogne (d'après Leroi-Gourhan, Allain, 1979 ; Breuil, 1985) ; plat néolithique à chauffer la saumure ?, Salaisons, Hérault (d'après Vaquer, 1975) ; coupes-à-socle chasséennes (d'après Thevenot, 1969) ; figuration d'une pièce tissée sur la stèle de Borno 1, face A, Valcamonica, Italie (relevé A. Fossati ; http://www.rupestre.it) preart.htm) ; tableaux de Kandinsky (d’après Henry, 1988) et Mondrian (d'après Jaffe, 1970) ; graffiti dans le centre-ville historique de Ljubjana, Slovénie (cliché : S. Cassen, CNRS) ; logo de la campagne «Sidaction, 2005 ».

Bien sûr, la «cité » biblique, la Jérusalem céleste a un plan carré (Apocalypse de Jean : 21-10) tandis que le jardin d'Éden est circulaire; mais n'entrons pas pour l'instant plus profondément dans l'évocation de ces deux intentions symboliques divergentes quant à la géométrie. Retenons plutôt, extraits du Livre des Morts égyptien (hymne à Osiris Oun-Néfer), les noms de Ouakh, Ousert et Qen-Qenet, ces bassins d'agrément toujours quadrangulaires, placés dans les «Champs Élyséens» ou "Champs des Roseaux», ce paradis des Bienheureux.

Un plan d'eau domestiqué dans un jardin de félicité, miniature quadrangulaire du monde idéal, voici un bon point de départ. Un commencement que prolonge aussitôt, si loin de là, à New York, un Central Park placé sur le nombril du monde d'une Big Apple paradisiaque et elle aussi fort tentatrice (fig. 16).

Sans développer une histoire hors sujet de ces portions d'espace, plans d'eau et plans de terre, évoquons néanmoins dans le cadre de la France classique la dimension spéciale que le jardin prendra par rapport au modèle italien, se muant en parc bientôt dit à la française. A. Le Nôtre (16131700) veut, à son propos, «élargir le cadre à l’infini ». Ici est situé le moment-clé, selon D. Le Couédic (1998), où l'on passa du registre privé à la sphère publique, par un nouveau recours à l'homothétie appelée à servir un changement d'échelle; une abstraction jouant sur un premier usage de cette transformation ponctuelle qui fit correspondre absolutisme royal et État national. Le souverain assume dès lors le passage du singulier au collectif ; autour de lui, des simulacres s'organisent, l'étiquette s'élabore, le paysage - physique et social - s'esquisse. La ville, extrapolation du château, singe la cour, et le jardin annonce le territoire national que les ingénieurs seront chargés de modeler à son image afin qu'il apparaisse à son tour comme un parc dessiné avec soin.

Cet effet de l'illuminisme universaliste et scientiste ( $i d$., p. 12) prit parfois l'allure d'un renouement avec la centuriation romaine, qui avait implacablement divisé l'Africa nova en parcelles carrées toutes identiques. Au point que le 29 septembre 1789, devant le Comité de Constitution, G. Thouret (1746-1794) proposa une départementalisation de la France en 100 carrés de 18 lieues de côté complétée par une subdivision en 900 cantons identiques, que l'on fût en plaine ou en montagne. La grille la plus stricte put ainsi s'imposer aux plans des villes : à Napoléon-Ville (aujourd'hui La Roche-sur-Yon en Vendée), ou encore dès la reconstruction de Lisbonne, en 1755, détruite par un tremblement de terre, et culminer à San Francisco, en 1849, dans le refus des contingences. Mais une telle grille réelle, de lecture et de projection sur une surface étendue, trouva également son emploi dans la division d'un continent, comme en témoigne le découpage de l'Amérique du Nord.

Un mouvement puissant était donc à l'œuvre, ajoute D. Le Couédic, qui visait à installer un système d'ordre universel, ouvrant l'époque du "paradis terrestre unifié ", expression empruntée à Robert Owen (1771-1858) qui, dans un XIX ${ }^{\mathrm{e}}$ s. renouant avec l'utopie, donnera, bien avant notre 


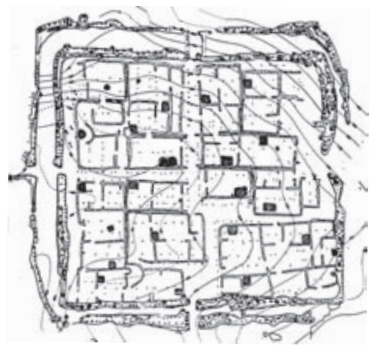

Polyanitsa, Bulgarie

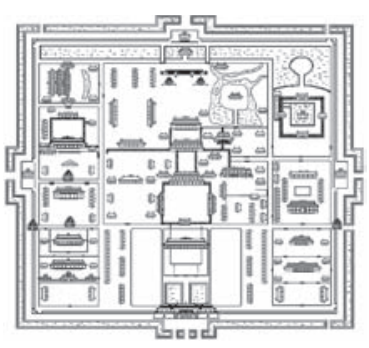

Hué, Vietnâm
HABITAT-HABITATION ET REPRÉSENTATION DE L'ESPACE
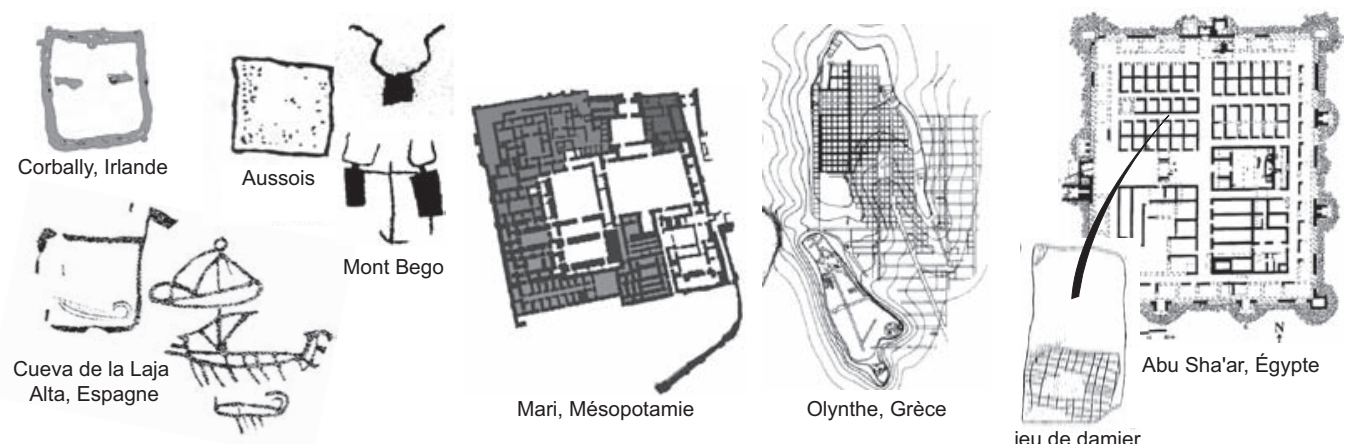

Abu Sha'ar, Égypte

jeu de damie
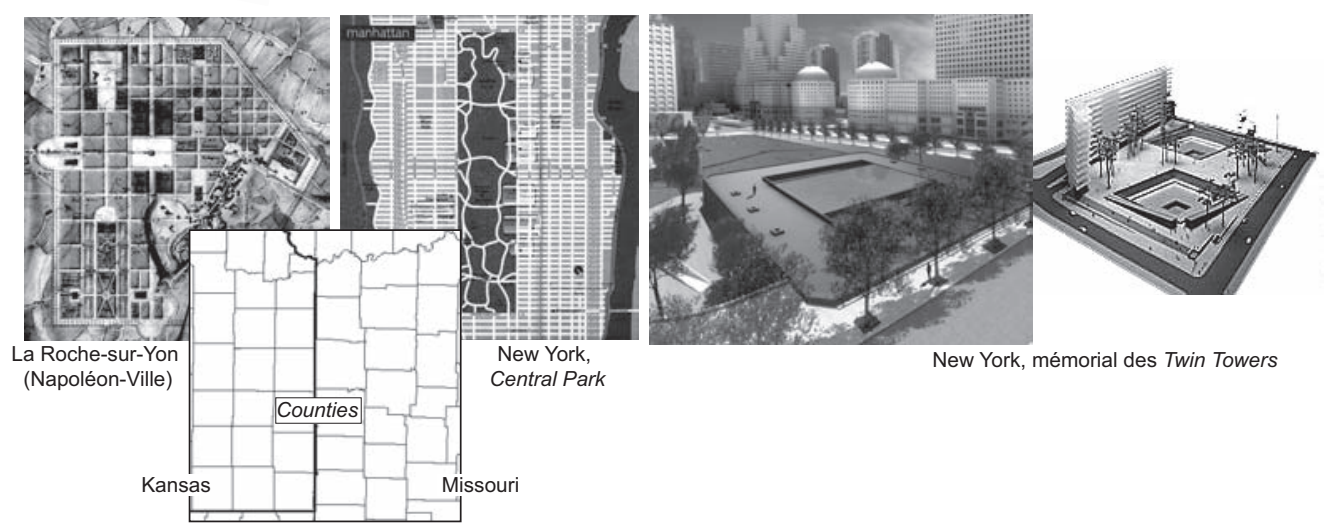

Fig. 16 - Représentation de l'espace à travers un raccourci de la maison quadrangulaire et son organisation villageoise, puis citadine : 7̌ niveau du tell néolithique de Polyanitsa, Bulgarie (d'après Todorova, 1978) ; maison néolithique de Corbally, Irlande (d'après Purcell, 2002) ; représentation d'un port et de bateaux antiques à la Cueva de la Laja Alta, Espagne, à 30 km de la mer (d'après Corzo Sanchez, 1983) ; gravure des Lozes à Aussois, Savoie (d'après Ballet, Raffaelli, 1991) et du Mont Bego, Alpes-Maritimes (d'après Lumley, Lautman dir., 1991) ; un plan de la ville mésopotamienne de Mari (d'après Margueron, 1982) ; plan de la cité grecque d'Olynthe (d'après Cahill, 2002) ; une des pierres gravées d'un jeu de damier trouvée dans le camp romain d'Abu Sha'ar, Égypte (d'après Mulvin, Sidebotham, 2004) ; plan de la ville d'Hué, Vietnâm (http://gilkergu.club.fr/hue/hue_cite_interdite/hueplan/plhue.htm); La Roche-sur-Yon, Vendée (plan Lamendé, 1804, $n^{o}$ d'inv. AN : F/14/10263/no 4, d'après une reproduction dans la revue Pays de Loire, $\left.n^{\circ} 303\right)$; carte des counties entre les états du Missouri et du Kansas, USA ; Central Park, le «jardin » inclus dans la cité quadrangulaire de New York; projet du lauréat du concours d'architecture pour le mémorial des Twin Towers, New York (http://www.wtcsitememorial.org/fin6.html).

moderne «mondialisation» dans la toile d'Internet, la description du «nouveau système universel » ${ }^{29}$, autrement dit la nouvelle Jérusalem ou paradis terrestre unifié, sous la forme d'une quadrangulaire cité unique ${ }^{30} \ldots$ Ou comment

29. «La terre sera disposée de telle façon qu'elle formera sur toute son étendue une seule cité composée de communes [...] chacune paradisiaque, reliée à toutes les autres communes du globe, jusqu'à ce que toute la Terre ne forme plus peu à peu que cette grande cité unique qu'on pourra appeler la nouvelle Jérusalem ou le paradis terrestre unifié ", Owen Robert, Robert Owen's Millenial Gazette, n II, $1^{\text {er }}$ août 1857, cité par Le Couédic, 1998.

30. La chaîne Euronews diffuse chaque jour un film introductif à son volet Economia dans lequel quelques carrés/parallélogrammes, égaux entre eux, peu nombreux et signifiant autant de pays, s'assemblent depuis l'espace pour former une grille parfaite sur l'ensemble du continent. appliquer un quadrilatère à la forme ronde de notre planète, vieille question de quadrature et de projection. La Vision de saint Paul (ou Apocalypse de Paul) donna d'ailleurs dans l'imagerie médiévale une urbanisation du Paradis poussée à son comble puisque le Paradis-Ville absorbe dans ses murailles le Paradis-Jardin et ses quatre fleuves arrosant les quatre coins du monde (Le Goff, 1985, p. 234).

Pour Platon, la géométrie (formes et raisonnements) est l'interface entre le monde matériel (l'homme et les objets terrestres) et ce qu'il appelle «les Idées d'essence divine». Dans Timée, le motif du carré est molécule du cube de la terre ; dans son Protagoras, il fait dire à Simonide de Céos de la difficulté à devenir cet homme carré, symbole du parfait et du divin... 
En Chine où le village néolithique de Shijiahe est une enceinte de terre formant un carré presque parfait de $1200 \mathrm{~m}$ de côté (Nakamura, 2005), on sait combien les études de M. Granet ont souligné cette constante millénaire dans les représentations chinoises du temps et de l'espace : le temps est rond, l'espace carré. L'empereur lui-même revêt un ample vêtement, rond au-dessus et carré au-dessous, qui représente l'univers dans sa totalité réuni autour de l'échelle de vie que constitue le corps impérial (Maertens, 1978, p. 52).

Les premiers villages mésopotamiens, en particulier le site de Nemrik sur la Djezireh irakienne (Huot, 1994, p. 49), nous disent le passage des abris circulaires de la phase ancienne, proches des huttes de la fin du $\mathrm{X}^{\mathrm{e}}$ millénaire, aux maisons de la phase moyenne, à poteaux puis à piliers, respectant encore un plan circulaire, pour aboutir à la maison, toujours à piliers, mais de plan quadrangulaire, de la fin de la phase récente. Une raison sans doute éloignée $\mathrm{du}$ seul progrès technique et qu'il faudra probablement rechercher du côté des quatre piliers divisant l'espace interne, centre du monde de l'homme sédentaire. Un homme qui, désormais, sillonne la terre avec ses outils et des animaux, en délimitant des surfaces quadrangulaires par des va-et-vient rectilignes. Des champs à angles droits représentés au flanc des montagnes alpines, comme une première topographie, ajoute A. Arcà (2004), dont la forme contamine si curieusement la gravure du corps carré ou rectangulaire des bœufs tirant l'araire du cultivateur sur ces mêmes rochers (fig. 16).

Plus tard, autour de cette proche Méditerranée, le premier élément de définition du temple des lointaines époques est assurément le plan ; il provient des archaïques formes quadrangulaires qui tendent petit à petit vers des proportions proches du carré ; il emprunte à la demeure aristocratique la distribution intérieure à trois pièces ou du moins divisée en trois espaces parallèles. Les rapports entre longueur et largeur, fixés par Vitruve, sont de 6/5 ; ils ne s'observent pas partout, mais semblent grossièrement correspondre à l'espace presque carré et strictement orienté que l'on nomme le templum et qui sert à l'observation des phénomènes du ciel (Jannot, 1998). Dans l'Italie antique, en effet, les augures procédaient à la prise des auspices par l'observation de signes célestes, comme le vol d'oiseaux à l'intérieur de ce templum, un carré isolé figurant le monde, et tracé dans le ciel à l'aide d'un doigt ou du lituus-crosse. Par extension, le camp militaire, monde déplacé, sera également planifié selon la méthode des augures (Lévy, 2003).
En comparaison, le jeu des 4 coins, motif répandu dans le folklore oral espagnol ${ }^{31}$, où il apparaît lié à une protection apotropaïque, semble d'une légèreté incomparable mais délivre bien ce qu'il veut signifier quant à la quadrature de l'habitation-refuge et de l'habitation-monde.

Tout aussi répétée est l'idée ancienne que l'on se faisait des îles ou des pays abstraits soutenus par quatre pieds ${ }^{32}$, rendant la forme bien stable, tandis que leur surface même sera divisée selon cette géométrie, à l'image de Belle-Île-enMer (Morbihan) ou de l'île d'Irlande ${ }^{33}$ dont G. Dumézil a rappelé que les quatre provinces qui, dans la géographie philosophique du pays, entourent le cinquième (cóiced) central, se définissent par l'excellence de leurs peuples respectifs dans le Savoir, le Combat, l'Abondance agricole et la Musique (Dumézil, 1985, p. 53).

Et quand il faudra finalement se souvenir des Twin Towers de la nouvelle Troie, édifiée elle aussi face à l'océan, tours réduites en poussière par l'irruption d'un monstre aérien et non plus sorti des eaux, un Léviathan inattendu alors qu'il avait été imaginé, vu, simulé, raconté par des mots et des images muettes puis parlantes depuis la fondation de la cité orgueilleuse, alors les hommes construiront, en creux, les deux grands motifs carrés symbolisant leur monde gémellé (fig. 16), mais qui fut soufflé dans ses fondations par un dieu aussi vengeur qu'Athéna, autrefois bataillant en Troade aux côtés des Achéens dresseurs de cavales.

Un monde dont le plus petit dénominateur commun, à l'heure où j'écris ces lignes et avec son aide, est un carré radical et minuscule (fig. 15), le pixel ${ }^{34}$ de toute notre

31. « Cuatros esquinitas/tiene mi casa/cuatro angelitos/que me la guardan/ San Juan, San Pedro, San Lucas, y San Mateo/Y la Viren Maria/que esta en el medio ». Merci encore à F. Delpech (CNRS) pour nous avoir communiqué cette comptine.

32. «Il y a une île lointaine/Alentour les chevaux de la mer brillent/ Belle course contre les vagues écumantes/Quatre pieds la supportent ", La Navigation de Bran, fils de Fébal, trad. de Georges Dottin.

33. Dans la description la plus complète et la plus authentique qu'est la Tain Bó Cualnge ou "Razzia des Vaches de Cooley ", l'Irlande est divisée en quatre royaumes, mais ceux-ci portent le nom de « cinquièmes » (cóiceda). Le cinquième royaume est connu à l'époque historique sous le nom de Mide, "le (royaume) du milieu» (Dillon, Chadwick, 1967, p. 43). Ces cinq provinces, toujours actuelles, sont : Connachta (Connaught), Ulaid (Ulster), Lagin (Leinster), Mumu (Munster) et Mide (le «milieu», Meath).

34. Une image «informatique» est constituée d'un ensemble de points appelés pixels (pixel est une abréviation de PICture ELement). Le pixel représente ainsi le plus petit élément constitutif d'une image numérique et l'ensemble de ces pixels est contenu dans un tableau à deux dimensions ; ils constituent une image en damier puisque l'écran effectue un balayage de gauche à droite et de haut en bas sur la surface de l'écran, un ordre choisi par l'homme, un ordre cartésien d'orientation dans l'espace à deux dimensions. On appréciera d'ailleurs à sa juste valeur emblématique la façon dont le film publicitaire 

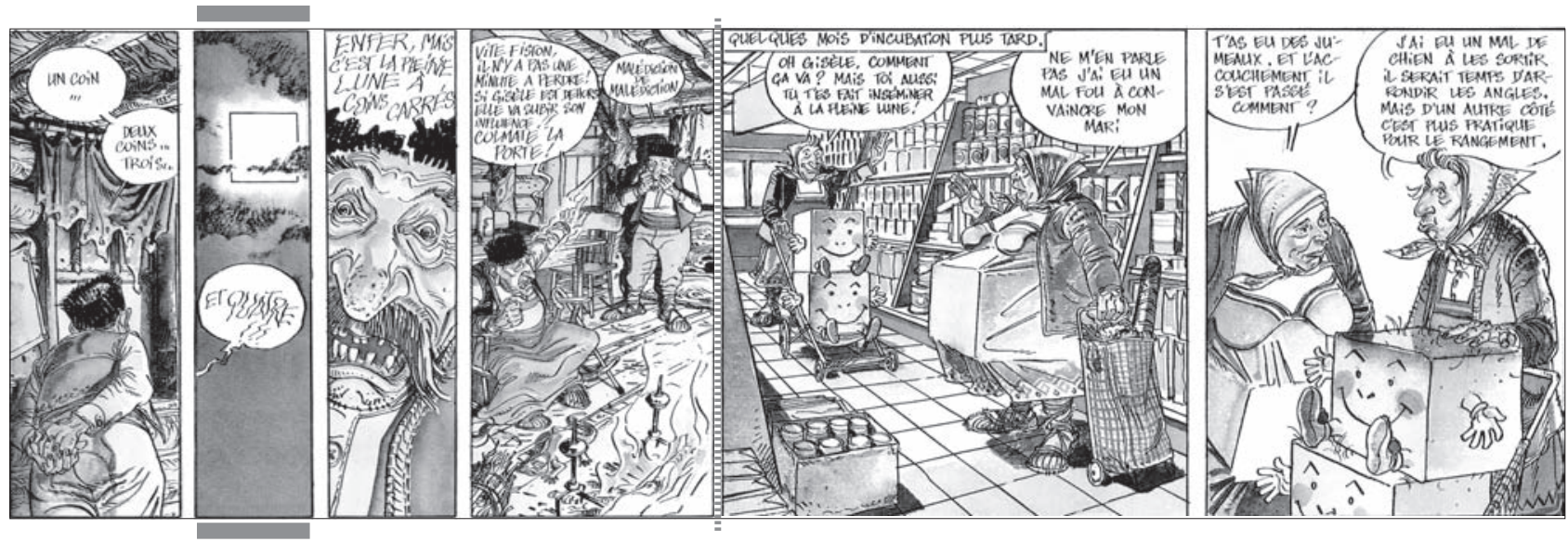

Fig. 17 - Linfluence de la lune carrée sur la libido des héros des Aventures de la Mort et de Lao-Tseu : la rage de vivre (Boucq, 1996; (C) Casterman, avec l'autorisation des auteurs et des éditions Casterman).

mémoire miniaturisée et, par suite, la représentation d'une planète enfin rectifiée et globalisée sous la toile en damier du joueur Palamède.

Poussé jusqu'à l'absurde, une situation imagée de notre quotidien laissera un carré prendre alors lieu et place du croissant-disque lunaire (Boucq, 1996) (fig. 17), un astre par ailleurs, comme on l'a vu, si prolifique de significations; un carré qui, par l'absurdité même du contexte, s'il paraît aussitôt insignifiant à la pensée rationnelle, n'en délivre pas moins la désignation poétique d'un monde en surface et en volume.

Cette forme gravée au Mané Lud et à Kermaillard, et ailleurs encore aux commencements du Néolithique, n'est donc pas celle d'un animal ni d'une divinité à figure (in)humaine.

L'objet susceptible de pouvoir subir autant d'impositions selon la taille (gigantesque), le milieu (marin) et la capacité à la miniaturisation n'est pas non plus un outil, ni une arme, mais l'espace délimité à la marge océane, l'abstraite surface, une terre, un monde, un jardin des délices, une arche, notre pays ou cette île fortunée vers laquelle cingle Bran le Corbeau ; peu importe.

( $1^{\text {er }}$ semestre 2005) de Bouygues Telecom, afin d'illustrer la technologie actuelle et pour assurer l'absolue nécessité d'une communication téléphonique, dématérialise le son issu d'une platine à disque vinyle en le divisant en une multitude de petits cubes, dématérialise par le même procédé des écrans de jeu vidéo et leurs consoles en damier noir et blanc, mais également et tout aussi bien les personnes dont visages et mains s'évaporent dans une même profusion nébuleuse de cubes, soit autant de pixels et de représentations des volumes sonores et visuels que va recomposer le génial petit téléphone portable usant, lui, en réalité, des ondes électromagnétiques et non de l'espace humain...

\section{SUR LA TERRE COMME AU CIEL}

Ainsi, comparer la cité coloniale grecque, ou le camp militaire romain, au village néolithique d'Europe centrale n'est pas sombrer dans l'incomparable. Tous trois subissent la loi des orients.

Ainsi, les cités chinoises des Shang (XVI ${ }^{\mathrm{e}} \mathrm{XIV}^{\mathrm{e}} \mathrm{s}$. av. J.-C.) sont caractérisées par le carré d'enceinte, l'orientation selon les points cardinaux, les plates-formes rituelles et les quartiers spécialisés. La religion codifie la conception et la réalisation.

Ainsi, en Mésopotamie, des règles rigides, des dimensionnements codifiés ayant pour bases des jeux de modules, fournissent cette similitude des plans carrés renvoyant à un système normalisé, à une codification des plans pour dire l'espace.

Ainsi, dans l'univers arabe, Ali Ibn Al-Husayn Al-Mas'udi nous rapporte que la terre-monde est divisée en quatre parties subissant les contraintes du temps qu'il fait, à l'instar de la vie de l'homme, comme l'édifice du corps humain reposant sur les humeurs cardinales, où l'est est sec et le nord bien froid... Et au centre du monde selon la religion islamique, cette construction de maçonnerie cubique dont les angles correspondent aux points cardinaux est la première création, la Kaaba, la Maison carrée sacrée par excellence ${ }^{35}$, édifiée par Adam, reconstruite par Abraham, autour de laquelle déambulent des millions de pélerins.

Comparer crosse et lituus n'est pas chuter dans l'incomparable puisque l'une et l'autre sont d'anciennes armes

35. Le Coran, sourate 5. La table servie (Al-Maidah) : 97: «Allah a institué la Kaaba, la Maison sacrée, comme un lieu de rassemblement pour les gens. " 
effectives, devenues objets mythologiques, insignes de statut, puisque l'une et l'autre sont par l'homme placées au contact d'une figure quadrangulaire :

- par l'augure étrusque cherchant une image de l'avenir en encadrant la portion fixe de ciel - extraction stabilisée si légère - de son évanescent lituus traçant le virtuel, attendant le passage ailé de l'oiseau en mouvement que jadis on frappait d'un boomerang, le vol d'une colombe ou de la corneille à la voix cassée ;

- par le graveur néolithique de l'Alentejo portugais dressant, sur une stèle statique et phallique, la crosse sur le côté du monde ramené à sa miniature minérale et pesante.

Comparer Locmariaquer, Saint-Michel-du-Touch et Varna, ce n'est pas subir seulement le sortilège analogique mais c'est aussi penser le Temps retrouvé :

- à travers la gravure rectangulaire, publique, placée en Morbihan au dos d'un phallus superlatif de crosses contenues et multipliées ;

- à travers la plaque quadrangulaire en pierre polie, suspendue au regard des hommes dans la vallée de la Garonne, mais à un seul individu destinée ;

- et à travers cette même miniaturisation portant la crosse en or aux côtés de la même forme géométrique, un quadrilatère, pour le profit d'un roi porteur d'une semblable histoire mythique, à l'autre extrémité de l'Europe en rivage d'une mer Noire.

Des formes, des objets, des bijoux qui restent seuls, parmi un ensemble qui nous sera à jamais éloigné parce que disparu, pour nous rappeler non seulement une représentation du monde d'autrefois, mais cette base essentielle des activités symboliques. Une force évocatrice, une telle pérennité, une telle extension communicatrice qui ont permis qu'à ce titre des objets et des figures deviennent ces emblèmes désirés et par conséquent un insigne du pouvoir que des humains habiles pourront magnifier au bénéfice des puissants.

Un temps retrouvé qui fut celui du temps deviné sur une surface imaginée.

\section{L'INTERROGATION DU SENS COMMUN}

Une consultation publique enrichit enfin la discussion. C'est un prolongement de l'expérience que nous avions déjà tentée à propos de la fameuse Déesse-Mère ${ }^{36}$ afin de conforter notre renversement de perspective au profit

36. Trois représentations de « Déesse-Mère " accompagnées de quinze couples de concepts antinomiques furent présentées en 1998 à 389 étudiants en psychologie. Une gradation en sept degrés permettait d'indiquer son opinion à propos de chaque doublet conceptuel. de l'image phallique (Cassen, 2000c); et avec le même succès dans l'étude du Mané Lud pour confronter le signe cornu à notre nouvelle acception en faveur d'un oiseau en plein vol (Cassen, 2005). Cette fois encore, on se propose d'approcher la perception d'un tracé en se référant au « sens commun » et à la spontanéité d'un public non spécialiste, à qui on laisse une totale liberté d'évocation, sans peur du ridicule apparent. La consultation, écrite et individuelle, porta en 2004 sur la promotion du Master 2 Pro nantais ( Les métiers de l'archéologie » dont la moyenne d'âge était de 25 ans) dont aucun des élèves ne connaissait les gravures bretonnes; l'interrogation se limita à présenter à chaque étudiant, sur une feuille A4, les deux motifs isolés de la stèle orientale du Vieux Moulin (carré penché + croissant) en sollicitant, en cinq ou six minutes, des réponses devant se résumer à quelques mots-clés, selon une suite de préférences croissantes. Les résultats furent par la suite discutés en cours (les étudiants sont désignés par leurs initiales, avec leur accord). Les interprétations sont données dans l'ordre de leur évocation ; les termes sont ceux-là mêmes qui furent employés, sans modification. Les fiches originales sont insérés au cahier de laboratoire, consultable à la demande (tabl. I).

On ne s'appesantira pas sur les indications «carré », ou " plan ", « ouverture ", « habitation, maison ", « terre ", qui tous renvoient à la perception d'un espace construit plus ou moins abstrait, plus ou moins réel, et dont les termes furent discutés au chapitre précédent. La tendance perceptive est conforme à notre renouvellement radical de l'image et interroge, de fait, le bien-fondé des perceptions univoques des spécialistes de l'art préhistorique.

Le second motif, placé sur la feuille, à droite du précédent, ainsi qu'ils sont ordonnés en réalité sur la stèle, est pensé soit isolément, abstraction faite du carré voisin, soit en rapport avec cette figure géométrique, alors que le premier motif semblait pouvoir fonctionner seul. Ce sont les commentaires formulés ensuite par les étudiants qui révélèrent cette différence de traitement et d'acceptation des figures, peut-être aussi contrainte par le sens usuel de lecture, de la gauche vers la droite.

Nous avons précédemment interrogé l'hypothèse « lune ", et nous n'y reviendrons pas, après avoir seulement noté la réponse de l'étudiant A. P. qui oppose deux astres, le soleil et la lune, réponse en soi fort cohérente malgré l'inadéquation géométrique de la forme solaire habituelle. Le terme « récipient, écuelle » n'est pas à rejeter a priori (et nous avons précédemment évoqué, en note, l'exégèse russe qui verrait la coupe de l'eucharistie dans le croissant accroché au pied de 
Tabl. I - Interprétations spontanées autour de deux figures gravées sur une stèle néolithique du Vieux Moulin (Plouharnel, Morbihan), isolées de leur contexte et de leur support. Enquête auprès de la promotion 2004 du Master 2 Pro "Les métiers de l'archéologie », université de Nantes.

\begin{tabular}{|c|c|c|}
\hline * & $\begin{array}{l}\text { 1're figure : } \\
\text { « carré penché » }\end{array}$ & $\begin{array}{l}2^{\mathrm{e}} \text { figure : } \\
\text { « croissant }\end{array}$ \\
\hline C. F. & carré & $\begin{array}{l}\text { lune } \\
\text { banane }\end{array}$ \\
\hline S. $\mathbf{R}$. & $\begin{array}{l}\text { ouverture } \\
\text { (fenêtre, porte, puits) }\end{array}$ & $\begin{array}{l}\text { lune } \\
\text { banane }\end{array}$ \\
\hline A. P. & $\begin{array}{l}\text { soleil } \\
\text { tête }\end{array}$ & $\begin{array}{l}\text { lune } \\
\text { bouche }\end{array}$ \\
\hline M. G. & $\begin{array}{l}\text { un plan } \\
\text { étoile }\end{array}$ & $\begin{array}{l}\text { pirogue } \\
\text { lune }\end{array}$ \\
\hline G. A. & carré & plat, coupe \\
\hline M. G. & base d'une habitation & $\begin{array}{l}\text { barque, bateau } \\
\text { une feuille }\end{array}$ \\
\hline C. $\mathbf{R}$. & plan de maison & $\begin{array}{l}\text { lune } \\
\text { écuelle }\end{array}$ \\
\hline \multirow[t]{2}{*}{ E. B. } & \multicolumn{2}{|c|}{ deux natures : rectilignes, curvilignes } \\
\hline & $\begin{array}{l}\text { en plan = espace délimité (maison ?) } \\
\text { profil =? }\end{array}$ & $\begin{array}{l}\text { en plan = quart de lune } \\
\text { en profil = bateau }\end{array}$ \\
\hline Y. D. & $\begin{array}{l}\text { terre } \\
\text { habitat (maison) }\end{array}$ & $\begin{array}{l}\text { bateau } \\
\text { coupe, récipient }\end{array}$ \\
\hline S. G. & rien & $\begin{array}{l}\text { croissant de lune } \\
\text { sourire } \\
\text { barque }\end{array}$ \\
\hline C. B. & bâtiment & quartier de lune \\
\hline A. C. & carré penché & $\begin{array}{l}\text { croissant de lune } \\
\text { piroque } \\
\text { bouche, sourire }\end{array}$ \\
\hline L. F. & carré & banane \\
\hline H. S. & sans réponse & sans réponse \\
\hline
\end{tabular}

* Initiales des participants au test.

la croix ${ }^{37}$ ), mais il ne s'inscrit pas dans la cohérence interne aux signes du Mané Lud, en particulier en regard des croissants surmontés de traits verticaux, longuement discutés dans un chapitre antérieur (voir supra, p. 225). Restent les termes «bouche, sourire, tête » qui doivent être analysés, puisqu'ils sont originaux. L'idée nous paraît en soi recevable. La coexistence, la contiguïté graphique sur l'écran de ces deux formes, quadrilatère et croissant, poussent nos jeunes observateurs à dévoiler une telle conclusion mentale, et l'on se doit de la déconstruire pour mieux plaider la cause inverse.

Il faut reconnaître en premier lieu qu'une tête peut être représentée carrée, non limitée à la ligne courbe (fig. 18). Mais il faut immédiatement ajouter qu'une tête est signifiée par des éléments graphiques aussitôt perceptibles, comme la bouche et les yeux ajoutés à la plaque murale taguée de

37. M. Cholokhov est également sensible à l'image du récipient (Le Don paisible, 1928-1940, p. 95) : «La coupe d'or de la jeune lune, pleine de bleu, était posée de travers dans le ciel [...]».
Ljubljana. À l'image du «cornu» réduit au bucrane qui doit, nous l'avons dit, nécessairement être reconnaissable à sa tête et à ses encornures, le visage n'a de sens que par ses attributs visuels caractéristiques, qu'un sourire, en effet, suffira à signifier (voir la publicité pour Badoit, etc.). Un sourire placé à distance d'une tête au carré perd son efficacité si ce dernier motif est réduit à sa seule ligne géométrique, vide, insensée. Un sourire, enfin, qui, figuré hors de toute liaison rapportée au visage, ne vaudra sa reconnaissance qu'au prix d'une référence à la physionomie humaine, en ajoutant pour cela les rides engendrées par cette contraction musculaire aux commissures des lèvres, suggérant ainsi le visage invisible qui le porte (fig. 18).

Ce n'est pas, en réalité, une découverte, et le Chat de Cheshire vous reviendra en mémoire... Alice a bien raison de ne pas vouloir d'un sourire sans sa tête porteuse ${ }^{38}$.

\section{IMAGES DU POUVOIR}

Voilà donc les signes de la Miniature et de l'Immensité...

Le grand sort du petit, écrivait Bachelard (1957, p. 145), non pas par la loi logique d'une dialectique des contraires, mais grâce à la libération de toutes les obligations des dimensions, libération qui est la caractéristique même de l'activité d'imagination.

Si nous suivons avec sympathie ce philosophe du sensible, mais aussi les sculpteurs de la pierre morbihannaise et les métallurgistes de la miniature bulgare, la contradiction géométrique est rédimée: la représentation est dominée par l'imagination. La représentation n'est plus qu'un corps d'expressions pour communiquer aux autres nos propres images.

«Le Monde est mon imagination... Je possède d'autant mieux le monde que je suis plus habile à le miniaturiser. Ce qui devient petit nous rend grands. »

En examinant les images au ralenti, en séjournant le temps nécessaire dans chaque image, on éprouve alors comme une coalescence de valeurs sans limites, des

38. « «Well! I've often seen a cat without a grin», thought Alice; «but a grin without a cat! It's the most curious thing I ever saw in all my life!» » (L. Carroll, 1865, Alice's Adventures in Wonderland). Les ambivalences/ équivalences que nous notions plus haut entre la lune, la faucille et la corne, se retrouvent augmentées d'un sourire ambigu dans le roman russe Le Don paisible, un sourire non pas isolé mais signifié par une peau diaphane colorée : «Un croissant de lune mince, tout mince, se profilait derrière un petit nuage rosissant, gai, comme le sourire d'une jeune fille. La fumée sortait toute droite de la cheminée et montait, forme sans bras, vers l'inaccessible lame d'or aiguisée de la lune déclinante. »(Cholokhov, 1928-1940, p. 228). 

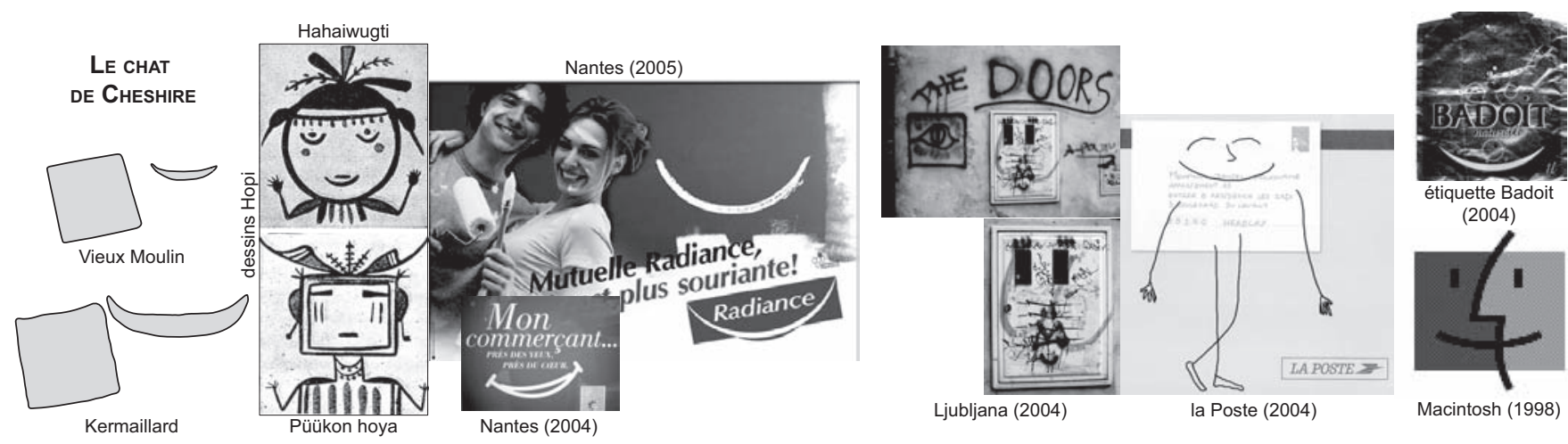

Fig. 18 - Traitement de l'hypothèse du "sourire » autour des motifs en croissant et quadrangulaires : exemples des stèles du Vieux Moulin à Plouharnel, Morbihan et Kermaillard à Arzon, Morbihan; représentation d'un visage, dessins sur poteries Hopi, USA : Hahaiwugti, femme de la Terre (en haut) et Püükon hoya, dieu de la petite Guerre (en bas) (d'après Kidder, Guernsey, 1919); publicité illustrant l'accueil souriant des commerçants (Nantes, 2005); graffitis dans une rue de Ljubljana, Slovénie, en 2004 (cliché: S. Cassen, CNRS) ; publicité pour la Poste, 2004 ; publicité pour l'eau pétillante Badoit qui rend de bonne humeur «Souriez avec Badoit »; logo du système MacOs 9.

valeurs qui s'engouffrent dans la miniature (id., p. 142). La miniature fait rêver. Entrez dans la miniature du Mané Lud et aussitôt les images se mettront à foisonner, à grandir, à s'évader. Une embarcation s'éloigne d'une terre et l'image poétique nouvelle devient ainsi, bien simplement, une origine absolue, une origine de conscience.

Mais point d'innocence en la matière : une fois compris son processus interne de "gullivérisation", l'image des commencements du Néolithique n'est pas la conscience unique d'une créativité individuelle, quand bien même le graveur au carré penché du Vieux Moulin (Plouharnel) rejoindrait la rêverie de K. Malevitch ${ }^{39}$ peignant en 1918 son Carré blanc sur fond blanc, oblique lui aussi et s'opposant au Carré noir de 1913 comme le pur mouvement à la station des choses possédées (fig. 13). Dans ces deux actes graphiques si semblables, seule la complexité extraordinaire du mouvement qu'il leur faut saisir est semblablement abstraite, et à chacun de nous rendue visible, et la solution d'une telle énigme enfin trouvée par un tracé "penché " redécouvert à plusieurs millénaires de distance, puisque tout l'Art est ensemble et que rien ne s'ajoute.

39. Malevitch adopte une trilogie pour les carrés selon leur couleur et les cite souvent groupés, dans le même ordre : noir, rouge, blanc (1920, p. 123) : «Les trois carrés suprématistes sont l'établissement de visions et de constructions du monde bien précises. [...] Dans la vie courante, ces carrés ont reçu encore une signification : le carré noir comme signe de l'économie, le carré rouge comme signal de la révolution, et le carré blanc comme pur mouvement (c'est nous qui soulignons) [...]. C'est la sensation de ce monde (sans objet absolu) qui brûle tous les vestiges de formes dans les deux pôles du suprématisme que sont le Carré noir et le Carré blanc.».
Car, bien différemment, le pouvoir de l'image à la Malevitch fait place, pour nous archéologues, aux origines de l'image du pouvoir en société. Tout d'abord, souvenonsnous avec M. Merleau-Ponty (1964), que le mot « image » est mal famé parce qu'on a cru qu'un dessin était un décalque, une copie, une seconde chose, et l'image mentale un dessin de ce genre dans notre bric-à-brac privé. Mais si, en effet, elle n'est rien de pareil, le dessin, la gravure et le tableau n'appartiennent pas plus qu'elle à «l'en soi ». Ils sont le dedans du dehors et le dehors du dedans - le quadrilatère dans le tableau rectangulaire, encadré (voir Magritte) -, que rend possible la duplicité du sentir, «et sans lesquels on ne comprendra jamais la quasi-présence et la visibilité imminente qui font tout le problème de l'imaginaire » (id., p. 23).

L'être de l'image n'est pas un moindre être, une deuxième chose en état de moindre réalité, renchérit à son tour L. Marin (1993, p. 10), et du même coup, en écran aux choses mêmes, un être d'illusion, un reflet appauvri, un voile trompeur, et d'autant plus trompeur que la relation de l'image à l'être se trouverait réglée par l'imitation qui ferait d'elle la représentation de la chose, doublant la chose et se substituant à elle.

Non, avec l'aide de ce chercheur nous devons nettement faire basculer la théorie de la représentation en Préhistoire du côté de l'examen de son efficacité sociale, lier la problématique du pouvoir à celle de l'image, un pouvoir qui sourd par toutes les entrées du jeu archéologique en Morbihan. Il faut pour cela, ensuite, bien concevoir l'accent que l'on doit mettre sur la force, le pouvoir de l'image substituée 
à une chose présente ailleurs. Cette compétition éternelle entre l'évocation de l'absence et l'exhibition de la présence.

Regardez les stèles funéraires de Pen Hape (Îleaux-Moines), du Manio 2 (Carnac), et les grandes stèles publiques - alignement du Grand Menhir (Locmariaquer), enceinte d'Er Lannic (Arzon) - voilà des lieux où penser la mort auprès des grands tombeaux d'Er Grah ou du Mané er Hroëck ; voici, entre cadavre-mort et message énoncé, I'énonciation puissante d'une absence - et c'est en cela que réside sa force pragmatique et historique, nous dit L. Marin (id., p. 11), son efficace fondatrice : l'absence du prestigieux corps fondateur.

Une absence du héros des commencements qui requerra sans cesse au long des siècles qu'il soit recouvert, inhumé, et comme monumentalisé, par et dans ses représentations.

Tel serait le premier effet de la représentation en général.

Mais comment l'image opère-t-elle cette transformation? D'un côté, elle met la force en état de signifiance « comme on met un bateau à l'eau » et, d'un autre, elle signifie «la force dans un discours, celui de la loi » (id., p. 14). Elle opère la substitution à la manifestation extérieure où une force n'apparaît que pour annihiler une autre force dans une lutte à mort, des signes de la force ou plutôt des signaux et des indices qui n'ont besoin que d'être vus, constatés, montrés, puis racontés et récités pour que la force dont ils sont les effets soit crue.

Des armes fulgurantes (hache, boomerang, arc/flèche), de gigantesques animaux (cachalot) et des bêtes angéliques (pigeon) ou réprouvées (corneille) sur les stèles morbihannaises... Voici l'image, effet-représentation dans et par ses signes, qui représente par traces et marques, la force : «Délégations de force, les signes ont moins valeur cognitive comme représentants de concepts que valeur pathique et esthétique comme signaux et indices » (id., p. 14) ; des représentants de force dans lesquels, à notre tour, nous les tard-venus, les "glosateurs", nous ne connaîtrons la force de l'image qu'en reconnaissant ses effets au titre des pouvoirs de l'image.

Dans la représentation qui est pouvoir, dans le pouvoir qui est représentation, le réel - le « réel » en tant qu'accomplissement toujours différé de ce désir (id., p. 16) - n'est autre que l'image fantastique dans lequel le sujet se contemplerait absolu.

Voilà le prestige des commencements, des fondations racontées et représentées, des ancêtres surnaturels à l'origine de l'ordre présent, des oppositions actuelles, de la légitimité du pouvoir détenu.

\section{D'UNE LISTE, L'AUTRE}

Au cours des 1500 premières années de l'histoire écrite de l'humanité, les textes sont le plus souvent, dans leur forme, très différents de la parole ordinaire, et même de toute parole. La plus caractéristique de ces formes n'apparaît que rarement dans le discours oral : c'est la liste (Goody, 1977, p. 148), série de noms ou de choses inscrits les uns à la suite des autres.

La liste du type le plus courant est une façon d'enregistrer des événements extérieurs, des rôles sociaux, des situations nouvelles ou à l'inverse bien établies, des personnes aussi, bien sûr ; les listes de rois en seraient l'exemple ancien très caractéristique. Il s'agit en quelque sorte d'inventorier une série de personnes, d'objets ou d'événements. Liste d'articles à écrire, liste pour aller faire ses achats, celle qui sert de guide, de plan à une action future, chaque article étant barré au fur et à mesure, mentalement ou réellement, et c'est alors l'itinéraire qui prend forme, tel celui qui permet de tracer la route à suivre pour se rendre en pèlerinage à La Mecque (id., p. 149) ou pour atteindre le lieu des Atlantes.

La liste implique discontinuité et non continuité. Elle suppose un certain agencement matériel, une certaine disposition spatiale, pouvant être lue en différents sens, latéralement et verticalement, de haut en bas comme de gauche à droite ; elle a un commencement et une fin bien marqués, une limite, un bord, tout comme une pièce d'étoffe, une arête de stèle ou la marge d'une diapositive consacrée au colloque du jour.

En effet, le terme "liste» en vieux français signifie aussi « le bord », « la bordure », « la lisière ", « la bande ", « le liseré », « le listel », « l'ourlet d'un vêtement », et très près de ce sens, on trouve celui de «limite» : ainsi, « entrer en lice » (to enter the lists en anglais, par emprunt au français ${ }^{40}$ ) signifie pénétrer dans l'aire délimitée pour le combat... L'idée d'une limite, ou plutôt de ce qui la rend plus visible, de ce qui la marque nettement, est alors un important attribut du genre de liste qui intéresse l'anthropologue, mais également l'archéologue, et dans ces termes, et ce sera la tâche du Mané Lud en mouvement que de faire interagir cette limitation aux signes gravés sur la stèle, en bordure du discours énonciateur dans notre propre discipline.

40. Dictionnaire TLFI (Atilf-CNRS-Nancy-II) : http://atilf.atilf.fr/tlf. $\mathrm{htm}$; noter également que la liste est aussi en vieux-français la bande de poils blancs sur le chanfrein des chevaux dont la robe est d'une autre couleur. 
Bref, et quand bien même l'importance prise par ces listes au Proche-Orient est en partie l'effet des besoins d'une économie et d'une organisation étatique, sous tous ces aspects la liste diffère de ce que produit la communication orale. Elle n'est pas la représentation directe de la parole, et elle travaille à la marge. Et s'il peut sembler vain de vouloir chercher dans l'écriture en tant que telle, ou dans cette suite de signes gravés, l'origine de toute hiérarchie ou de toute domination, on pourra néanmoins s'interroger avec nos collègues, ici sur le rôle de l'écriture, là sur des agencements de signes gravés «alignés" sur l'écran minéral, dans la transformation du savoir politique, dans la mise en place de nouveaux modes de pensée correspondant à de nouveaux modes de domination que reflètent à nos yeux l'érection en un temps si court d'ouvrages parmi les plus gigantesques du $V^{e}$ millénaire européen. Car les premiers systèmes d'écriture, et même ces procédés graphiques plus anciens qui jalonnent l'histoire gravée d'un commencement, ont influencé à la fois l'organisation de la vie sociale et celle des systèmes de connaissance (Goody, 1977, p. 142).

Voici la prouesse technique poussée par la raison symbolique, des centaines de tonnes de pierres transférées en bord d'océan, en limite de l'inconnaissable, pour servir de miroir mémorable au mythe, peut-être celui d'une origine, de la fondation d'un roi. Et quand bien même l'on ne peut pas réduire un message au moyen matériel de sa transmission, tout changement dans le système des communications - et la «culture » archéologique est aussi cet enchaînement d'actes de communication - a nécessairement en retour d'importants effets sur les contenus transmis ${ }^{41}$.

Ceci est le Néolithique nouveau en sa révolution occidentale.

En résumé, deux listes d'images sont en présence et en compétition au Mané Lud :

- la Grande Déesse : un cachalot soufflant ;

- l'herminette : un homme bras en croix ;

- la hache du bûcheron : une arme guerrière ;

- la houlette du berger : un bâton de jet ;

- le cornu : un oiseau volant ;

- le peigne-graffiti : un bateau monté ;

- l'idole en écusson : un monde en miniature.

Voici ma liste d'images à vivre sincèrement, des images qu'un esprit positif et expérimenté peut sans doute juger comme issues de pensées folles, mais dont on est bien obligé de reconnaître la cohérence archéologique, la cohérence

41. Voyez le code informatique qui transmet le texte, les nombres et l'image, l'extraordinaire bouleversement de ces médiations que nous ne mesurons pas encore puisque nous le vivons au jour le jour. des «vieilles histoires", cette archaiologia selon Plutarque, et non celle des propos rebattus (archaiologeîn). Elles ont précisément la cohérence bachelardienne de l'imagination matérielle qui ne se laisse pas détourner par les images diverses de la forme et de la couleur, mais qui rêve à la substance, aux forces profondes de la substance, aux vertus du monde concret.

Cette cohérence n'est pas non plus celle d'un dogme ; on tente ici de ne pas se prendre au piège de la fascination pour la cohérence interne, le premier postulat de l'interprétation, ce faux concept de l'interprétation historienne (Détienne, 1981, p. 58), qui se borne à dire l'absence de disparité entre les parties d'une doctrine ou d'un ouvrage - des gravures de bêtes et d'instruments agricoles faites par des fermiers, pour des fermiers -, sans autre répondant que son opposé, l'incohérence, ce «presque synonyme de folie ».

\section{IMAGE DE FIN}

En suivant l'idéal d'intellectualisation, nous, archéologues, pensons encore que nous aiderions à comprendre la légende si nous retrouvions les plans de la construction de Dédale, le mythe morbihannais de saint Corneille si nous découvrions sa tombe très chrétienne, ou l'aspect originel de Carnac si le paysage néolithique exact était enfin reconstitué. Mais, disent les philosophes (Bachelard, 1948, p. 210), si utiles que soient les recherches des faits, pas de bonne archéologie historique sans une archéologie "psychologique » des images. Toutes les œuvres claires ont une marge d'ombre.

Il est vrai que les choses sont ici bien transcendantes, c'est-à-dire que je ne les possède pas, que je n'en fais pas le tour, dans la mesure où j'ignore ce qu'elles sont mais où j'en affirme aveuglément l'existence nue... Qui plus est, bien des hommes sensibles ont déjà noté l'extrême différence existant entre l'impression vraie que nous avons eue d'une chose (le goût d'une madeleine, l'écoute d'une sonate, la vue du souffle d'un cachalot) et l'impression factice que nous nous en donnons quand volontairement nous essayons de nous la représenter. À vrai dire, tout ce que nous pouvons faire, c'est nous « rappeler » le passé, lui redonner une existence idéale, car la reconstruction idéale en ce domaine de la représentation, et non l'observation empirique, constitue la première étape de la connaissance historique (Cassirer, 1975, p. 246).

Ce que nous avons trouvé au Mané Lud n'est donc pas une suite éperdue de graffitis, ni une liste d'objets physiques, ni même le reflet de l'état économique d'une 
société agropastorale, mais un univers symbolique qu'il nous faut apprendre à redire avec tranquillité, sans mépris, sans paresse. Tout fait historique, aussi simple puisse-t-il paraître, ne peut être déterminé et compris que par une telle analyse préalable des symboles. Et ce, en enregistrant dans la patiente urgence des documents fugaces, des images qui prennent progressivement forme. Des images, on le verra, qui pourraient bien être à la fois l'instrument de la force, le moyen de la puissance et sa fondation en pouvoir.

Il apparaît, pour finir, un éloignement imaginaire du passé dont nous sommes dupes dans cette rationalisation archéologique. Le passé non seulement n'est pas si fugace, mais il reste sur place. Les mots ne changent pas tant de signification pendant des siècles que pour nous les noms dans l'espace de quelques années. Nous n'avons pas assez de place, dans notre pensée actuelle, pour y garder les morts à côté des vivants. Nous sommes alors obligés de construire sur ce qui a précédé et que nous ne retrouvons qu'au hasard d'une fouille, racontait Marcel Proust (1920, p. 405), du genre de celle que les mots de Corneille ou de Morvil viennent de provoquer en Bretagne méridionale, sans pelles ni râteaux... 


\section{BIBLIOGRAPHIE}

\section{AbÉlanet J.}

1990 : Les Roches gravées nord-catalan, Prada, (coll. Centre d'études préhistoriques catalans, 5).

ADAMS L.

1984 : Les Peintures rupestres du Levant espagnol, Paris, Picard.

AL-HusaYn AL-Mas'Udi A. (947)

1997 : Les Prairies d'or, Paris, Paul Geuthner.

ALPS S.

1947: "La désignation du Lituus en hittite ", Journal of Cuneiform Studies, 1, 2, p. 164-175.

\section{ANATI E.}

1996: La Civiltà della Valcamonica, Milano, Il Saggiatore.

ANati E., RUStamov D. N., MURadova F., FARADJEVA M.

2001: Gobustan, Azerbaijan, Capo di Ponte, ed. del Centro, Wara Archives.

\section{ARCÀ A.}

2004 : « The topographic engravings of Alpine rock-art: fields, settlements and agricultural landscapes ", in CHIPPINDALE C., NASH G. (DIR.), The Figured Landscapes of Rock-Art. Looking at Pictures in Place, Cambridge, Cambridge University Press, p. 318-349.

ASHE G.

1990 : The Mythology of the British Isles, Londres, Methuen London.

\section{BACHELARD G.}

1933: "Le monde comme caprice et miniature ", Recherches philosophiques, III, p. 306-320.

1943 (rééd. 1992) : L’Air et les songes, Paris, Librairie José Corti (coll. Biblio essais).

1947 : La Terre et les rêveries de volonté : essai sur limagination de la matière, Paris, librairie José Corti.

1948: La Terre et les rêveries du repos : essai sur les images de lintimité, Paris, Librairie José Corti.

1957 (rééd. 1994) : La Poétique de l'espace, Paris, PUF.

1960 (rééd. 1993) : Poétique de la rêverie, Paris, PUF (coll. Quadrige).

\section{BALlet F., RAFFaElli P.}

1991: «Gravures figuratives et abstraites des âges des métaux dans les Alpes de
Savoie ", in Lumley H. DE, Lautman F. (DIR.), Le Mont Bego: une montagne sacrée de l'âge du Bronze, Paris, Institut de Paléontologie humaine, p. 162-191.

\section{BARTHES R.}

1957 (rééd. 1970) : Mythologies, Paris, Le Seuil

\section{BAUDAIS D.}

1985: «Le mobilier en bois des sites littoraux de Chalain et Claivaux ", in Chalain-Clairvaux fouilles anciennes, Lons-le-Saunier, Musée archéologique (coll. Musée archéologique, 1), p. 177 199.

\section{BAZZANELla M.}

1997: «Les vases à ouverture carrée en Europe occidentale", in La Culture de Cerny: nouvelle économie, nouvelle société au Néolithique, Actes du colloque international, Nemours 1994, Nemours, Musée de Préhistoire (coll. Mémoires du musée de Préhistoire d'île-de-France, 6), p. $557-575$.

\section{BEBRE V.}

2003: «Drinking horns in Latvia (3 ${ }^{\text {rd }}$ $13^{\text {th }}$ century) ", Humanities and Social Sciences, Latvia, 2 (39), p. 18-35.

\section{BEFFA M.-L., DELABY L.}

1999: Festins d'âmes et robes d'esprits: les objets chamaniques sibériens du musée de l'Homme, Paris, Publications scientifiques du Muséum d'histoire naturelle (coll. Ethnologie, 181), $242 \mathrm{p}$

\section{BEHRENS H.}

1973: Die Jungsteinzeit im Mittelelbe-SaaleGebiet, Berlin (coll. Veräffentlichungen Landesmuseum für Vorgeschichte in Halle, 27).

\section{BELLANCOURT G.}

1977: «Étude des figurations glyptiques observées en Loire-Atlantique -I- Les rochers gravés du Méniscoul, Piriac-surMer (Loire-Atlantique) ", Bulletin de la Société nantaise de Préhistoire, Études 2, p. 1-39.

\section{BEHRENS H.}

1973: Die Jungsteinzeit im Mittelelbe-SaaleGebiet, Berlin (coll. Veräffentlichungen Landesmuseum für Vorgeschichte in Halle, 27). (coll. Points Essais)

\section{BERTRANDY F., SZNYGER M.}

1987: Les Stèles puniques de Constantine, Paris, éd. de la Réunion des Musées nationaux, Musée du Louvre (notes et documents).

\section{BEST E.}

1934: The Maori as he Was : a Brief Account of Maori Life as it twas in Pre-European Days, Wellington, New Zealand, Dominion Museum (http://www.nzetc.org/tm/ scholarly/tei-BesMaor.html).

\section{BITTEL K.}

1976 : Les Hittites, Paris, Gallimard (coll. l'Univers des Formes).

BOAS F.

1883 : The Central Eskimo. Hunting E Fishing; Seal, Walrus \& Whale Hunting. Ethnological Results of the Point Barrow Expedition by John Murdoch, naturalist and observer, international Polar Expedition to Point Barrow, Alaska, 1881-1883, Smithsonian Institute, Washington, Bureau of Ethnology.

\section{BOTTERO J.}

1987 : Mésopotamie, l'écriture, la raison et les dieux, Paris, Gallimard (coll. Folio Histoire).

\section{BOUCQ}

1996 : Les Aventures de la mort et de Lao-Tseu : la rage de vivre, Tournai, Casterman.

\section{Boujot C., CASSEN S.}

1992: «Le développement des premières architectures funéraires monumentales en France occidentale ", in Paysans et bâtisseurs : l'émergence du Néolithique atlantique et les origines du mégalithisme, Actes du XVII colloque interrégional sur le Néolithique, Vannes, 2931 oct. 1990, Rennes (coll. Suppl. à la Revue archéologique de l'Ouest, 5), p. 195-211.

Boujot C., Cassen S., Defaix J.

2000: «La pierre décorée du caveau et les gravures régionales nouvellement découvertes", in Éléments d'architecture (exploration d'un tertre funéraire à Lannec er Gadouer, Erdeven, Morbihan. Constructions et reconstructions dans le Néolithique morbihannais. Propositions pour une lecture symbolique), Chauvigny, éd. chauvinoises (coll. Mémoire, 19), p. 279-298.

\section{Boujot C., Cassen S., Vaquero}

LASTRES J.

1995 : « Ideas de tierra », in Actas VI, Congresso de arqueologia Péninsular, Porto 1993, Porto, 
Sociedade portuguesa de antropologia e etnologia, 2, p. 169-191.

1998: «Some abstraction for a practical subject: the neolithization of Western France as seen through funeral architecture data", Cambridge Archaeological Journal, 8 (2), p. 193-206.

\section{BOYER P.}

2003: Et l'homme créa les dieux: comment expliquer la religion, Paris, Gallimard (coll. Folio Essais).

\section{BRADLEY R.}

1989: "Dead and entrancess: a contextual analysis of megalithic art », Current Anthropology, 30, p. 68-75.

\section{BREUIL H.}

1934-1935: Les Peintures rupestres schématiques de la péninsule Ibérique, III et IV, Paris, Fondation Singer-Polignac, Imprimerie de Lagny.

1985: Quatre cents siècles d'art pariétal: les cavernes ornées de l'âge du renne, Paris, M. Fourny.

\section{BREUIL H., BOYLE M.-E.}

1959: Quelques dolmens ornés du Morbihan: essai de déchiffrement de leurs décorations, Paris, PUF (coll. Préhistoire, XIII).

\section{BRIARD J.}

1987 : Mythes et symboles de l'Europe préceltique: les religions de l'âge du Bronze (2500-800 av. J.-C.), Paris, éd. Errance (coll. les Hespérides).

1995: Les Mégalithes de l'Europe atlantique: architecture et art funéraire (5000-2000 av. J.-C.), Paris, éd. Errance.

\section{BRøNDSTED J.}

1957: Danmarks Oldtid, Copenhagen, Gyldendal.

\section{CAHILl N.}

2002: Housing and City Organization at Olynthus, New Haven, Yale University Press.

\section{Calado M.}

1997: "Cromlechs alentejanos e arte megalitica", in $I I^{e}$ Colloquio intern. de Arte megalitico, A Coruña, 1997, A Coruña (coll. Brigantium, 10), p. 289-297.

2002: «Standing stones and natural outcrops: the role of ritual monuments in the Neolithic transition of the Central Alentejo », in SCARRE C. (DIR.), Monuments and Landscapes in Atlantic Europe, London, New York, Routledge, p. 17-35.
2004: «Entre o Ceu e a Terra. Menires e arte rupestre no Alentejo central ", in $1^{\circ}$ International Colloquium on Rock Art and Megalithism in Atlantic Europe, Sinais de Pedra, Evora, 24-26 janv. 2003, Fundaçao Eugenio da Almeida, Publication sur cédérom.

\section{Calvet J.-L.}

1996 : Histoire de l'écriture, Paris, Plon.

\section{Cantalejo P., Maura R., Becerra M.}

2006 : Arte rupestre prehistórico en la Serranía de Ronda, Ronda (Malaga), La Serranía.

\section{CARENOU C}

1935 : La Religion dolménique, suivie du déchiffrement de la pierre du dolmen ruiné de Parc Guren, Paris, Librairie Paul Geuthner, 92 p., 25 gravures.

\section{CARPENTIER V., GHESQUiÈre E.,}

\section{MARCIGNY C.}

2002: Graffiti marins des églises du Val de Saire: Réville, Quettehou, Morsalines, Musée maritime de l'île de Tatihou.

\section{CARRÉ H.}

1996: "Passy (Yonne) et sa céramique: sites habités et sépultures", in Actes $d u$ XVIII colloqueinterrégionalsurle Néolithique, Dijon 1991 (coll. Suppl. à la Revue archéologique de l'Est, 14), p. 307-338.

\section{Cassen S.}

1993: «Material culture and chronology of the Middle Neolithic of Western France ", Journal of Archaeology, 12, 2, p. 197-208.

1999: "Questions of epistemology and a working hypothesis about engravings of the $5^{\text {th }}$ millenium in Western France", in "Theory in French Archaeology", Antiquity, 73 (279), p. 198-205.

2000a : «Du réemploi des dalles gravées à la sexualisation des signes: petite histoire de la recherche ", in Éléments d'architecture (exploration d'un tertre funéraire à Lannec er Gadouer, Erdeven, Morbihan. Constructions et reconstructions dans le Néolithique morbihannais. Propositions pour une lecture symbolique), Chauvigny, éd. chauvinoises (coll. Mémoire, 19), p. 593-610.

2000b: «Funerary stelas reused in the passage graves of Western France: history of research and sexualisation of the carvings", in RITCHIES A. (DIR.), "Orkney (Écosse)», Neolithic Orkney in its European Context, Kirwall 1998, Cambridge, McDonald Institute Monographs, p. 233-246.

2000c : « La forme d'une déesse », in Éléments d'architecture (exploration d'un tertre funéraire à Lannec er Gadouer, Erdeven, Morbihan. Constructions et reconstructions dans le Néolithique morbihannais. Propositions pour une lecture symbolique), Chauvigny, éd. chauvinoises (coll. Mémoire, 19), p. $657-672$.

2000d: "Architecture du tombeau, équipement mortuaire, décor céramique et art gravé du $\mathrm{V}^{\mathrm{e}}$ millénaire en Morbihan: à la recherche d'une cosmogonie des premières sociétés agricoles de l'Europe occidentale ", in Actas do $3^{\circ}$ congresso de arqueologia peninsular-IV-Pré-historia recente da Peninsula ibérica, Porto, ADECAP, p. $447-479$.

2000e : "La crosse, lacrosse», in Éléments d'architecture (exploration d'un tertre funéraire à Lannec er Gadouer, Erdeven, Morbihan. Constructions et reconstructions dans le Néolithique morbihannais. Propositions pour une lecture symbolique), Chauvigny, éd. chauvinoises (coll. Mémoire, 19), p. 673-678.

2000f : « La fabrication du sel : une hypothèse fonctionnelle pour la forme céramique du caveau de Lannec er Gadouer ", in Éléments d'architecture (exploration d'un tertre funéraire à Lannec er Gadouer, Erdeven, Morbihan. Constructions et reconstructions dans le Néolithique morbihannais. Propositions pour une lecture symbolique), Chauvigny éd. chauvinoises (coll. Mémoire, 19), p. 249-265.

2000g: "Terminaison", in Éléments d'architecture (exploration d'un tertre funéraire à Lannec er Gadouer, Erdeven, Morbihan. Constructions et reconstructions dans le Néolithique morbihannais. Propositions pour une lecture symbolique), Chauvigny, éd. chauvinoises (coll. Mémoire, 19), p. $737-752$.

2003 : «Importer, imiter, inspirer ? Objetssignes centre-européens dans le Néolithique armoricain ", L'Anthropologie, 107, p. 255-270.

2004: «La navigation de pierre: une représentation du voyage sur les stèles armoricaines du $\mathrm{V}^{\mathrm{e}}$ millénaire ", in XXI Valcamonica Symposium: nuove scoperte, nuove interpretazioni, nuovi metodi di ricerca, Arte preistorica e tribale, Darfo Boario, Centro camuno di studi preistorici, p. 148-154.

2005 : «Pigeon-vole ! Re-connaissance d'une gravure armoricaine du Ve millénaire", Bulletin de la Société préhistorique française, 102, 2, p. 299-334.

2007: «Un pour tous, tous contre un... Symboles, mythe et histoire à travers une stèle morbihannaise du $\mathrm{V}^{\mathrm{e}}$ millénaire ", in Testart A., BARrayJ.-L., BRUN P. (DIR.), Pratiques funéraires et sociétés: nouvelles approches en archéologie et en 
anthropologie sociale, Actes du colloque de Sens, 12-14 juin 2003, Dijon, Université de Dijon (coll. Art, archéologie et patrimoine), p. 37-67.

Cassen S., LABriffe P.-A. DE, MENANTEAU L.

2004: «Sels de mer, sels de terre: indices et preuves de fabrication du sel sur les rivages de l'Europe occidentale, du $\mathrm{V}^{\mathrm{e}}$ au III millénaire ", Cuadernos de Arqueologia, 12 , p. $9-49$.

Cassen S., Lefebvre B., Merheb M.

2005: "Mané Rutual en Locmariaquer (Morbihan) : enregistrement de gravures et simulations architecturales dans une tombe à couloir néolithique", Revue archéologique de l'Ouest, 22, p. 93-124.

Cassen S., Lefebvre B., VaQuero J., COLLIN C.

2005: "Le Mané Lud en sauvetage (Locmariaquer, Morbihan): enregistrement et restitution de signes gravés dans une tombe à couloir néolithique », L'Anthropologie, 109, p. 325-384.

\section{CaSsen S., Merheb M.}

2004: "Archiving Neolithic heritage ", in The Standing Appointment for Professionals in 3D Scanning Solutions, International Conference 3rdDiMENSIon, Paris, p. 13-26 [publication sur cédérom].

CASSEN S., VAQUero J.

2000 : «La forme d'une chose », in Éléments d'architecture (exploration d'un tertre funéraire à Lannec er Gadouer, Erdeven, Morbihan. Constructions et reconstructions dans le Néolithique morbihannais. Propositions pour une lecture symbolique), Chauvigny, éd. chauvinoises (coll. Mémoire, 19), p. 611-656.

2003 : "Le désir médusé ", in Guilaine J. (DIR.), Expressions symboliques, manifestations artistiques du Néolithique et de la Protohistoire, Séminaires du Collège de France, Paris, éd. Errance, p. 91-118.

\section{CASSIRER E.}

1924 (rééd. 1972) : La Philosophie des formes symboliques -2- La Pensée mythique, Paris, éd. de Minuit.

1975 : Essai sur l'homme, Paris, éd. de Minuit (coll. Le Sens commun)

\section{Gerrato Leyton F., Novillo \\ GONZALEZ V.}

2000: «Los petroglifos de la rivera del Guadiana a su paso por las inmediaciones de Cheles (noticia arqueologica)",
Revista de estudios extremeños, Badajoz, Centro de estudios extremeños, p. 91-98.

\section{CHARACHIDZÉ G.}

1994 : «Ecriture de l'histoire géorgienne, ou la mythologie travestie », in DÉTIENNE M. (DIR.), Transcrire les mythologies, Paris, Albin Michel (coll. Idées), p. 203-228.

\section{Chataignier C.}

1995: La Transcaucasie au Néolithique et au Chalcolithique, Oxford, Archaeopress (coll. British Archaeological Reports, International Series, 624).

\section{Cherry J. C.}

1981 : «Pattern and process in the earliest colonization of the mediterranean islands ", Proceedings of the Prehistoric Society, 47, p. 41-68.

\section{Chevallier J., Gheerbrant A. (DIR.)}

1969 : Dictionnaire des symboles: mythes, rêves, coutumes, gestes, formes, figures, couleurs, nombres, Paris, Robert Laffont.

\section{Chocheyras J.}

1997: Saint Jacques à Compostelle, Rennes, Ouest-France.

\section{Cholokhov M.}

1928-1940: Le Don paisible, T1, T6, Paris, R. Julliard, trad. A. Vitez， 1959 (t. 1), 1962 (t. 2).

\section{Closmadeuc G. DE}

1873: Sculptures lapidaires et signes gravés des dolmens dans le Morbihan, Vannes, Imprimerie de Lamarzelle.

COLES J.

2004: "Bridge to the outer world: rock carvings at Bro Utmark, Bohuslän, Sweden ", Proceedings of the Prehistoric Society, 70, p. 173-206.

\section{Collado Giraldo $\mathrm{H}$.}

2004: «Un nuevo ciclo de arte prehistorico en Extremadura : el arte rupestre de las sociedades de economa cazadora recolectora durante el Holoceno inicial como precedente del arte rupestre esquematico en Extremadura », in $l^{\circ}$ International Colloquium on Rock Art and Megalithism in Atlantic Europe, Sinais de Pedra, Evora, 24-26 janv. 2003, Fundaçao Eugenio da Almeida [publication sur cédérom].

\section{CORZO SANCHEZ R.}

1983 : «Cádiz y la arqueología fenicia », Anales de la real academia provincial de Bellas Artes de Cádiz, 1, p. 5-29.
Crewe L., Peltenbrug E., Spanou $\mathbf{S}$.

2002 : «Contexts for cruciforms : figurines of prehistoric Cyprus », Antiquity, 76, 291, p. 21-22.

\section{DANTE}

1321 : Euvres italiennes, ouvres latines et La Divine comédie, trad. A. Pézard, 1988, Paris, Gallimard.

DAVY DE CUSSÉ L.

1865-1866 (2 ${ }^{\mathrm{e}}$ livraison): Recueil des signes sculptés sur les monuments mégalithiques $d u$ Morbihan relevés et réduits au pantographe, Vannes, Imprimerie Galles.

\section{DÉCHELETTE J.}

1908-1928 (rééd. 1992) : Manuel d'archéologie préhistorique et celtique -1-Archéologie préhistorique, Paris, Picard.

\section{DEFAIX J.}

2001: «Mégalithes et espace: pour une approche anthropologique », 7, in DUHAMEL P. (DIR.), Actes du $25^{e}$ colloque interrégional sur le Néolithique, Dijon, Service régional de l'archéologie de Franche-Comté [résumés des communications p. II]

\section{DELAISSE L. M. J.}

1959: Miniatures médiévales de la librairie de Bourgogne au cabinet des manuscrits de la Bibliothèque royale de Belgique, Bruxelles, éd. de la Connaissance.

\section{DÉTIENNE M.}

1981: L'Invention de la mythologie, Paris, Gallimard (coll. Tel).

1998: Apollon le couteau à la main, Paris, Gallimard.

Dillon M., Chadwick N. K

1967 (rééd. 1979) : Les Royaumes celtiques (The Celtic Realms), Paris, Marabout (coll. Université).

\section{Diserens C., Chevrier J.-F.}

2003 : Marcel Broodthaers, Paris, éd. Images En Manœuvres.

DJAFARZADE N. M.

1973 : Gobustan (en russe), Baku, s. éd.

DORFER, L., MOSER M., SPINDLER K., BAHr F., EGARTER-Vigl E., DOHR G. 1998 : « 5200-year-old acupuncture in central Europe? », Science, 282, p. 242--243.

\section{DOURY C.}

1999 : Peuples de Sibérie, du fleuve Amour aux terres boréales, Paris, Le Seuil. 


\section{DRAGAN R.}

1999 : La Représentation de l'espace de la société traditionnelle: les mondes renversés. Paris, L'Harmattan (coll. Connaissance des hommes)

\section{DUMÉZIL G.}

1971 (rééd. 1995) : Mythe et épopée -III- Types épiques indo-européens : un héros, un sorcier, un roi, Paris, Gallimard (coll. Quarto).

1985 : L'Oubli de l'homme et l'honneur des dieux, et autres essais : vingt-cinq esquisses de mythologie (51-75), Paris, Gallimard.

DURAND G.

1969 (rééd. 1992): Les Structures anthropologiques de limaginaire, Paris, Dunod.

\section{EBBESEN K.}

1975: Die jüngere Trichterbecherkultur aud den dänischen Inseln, Copenhagen, Arkaeologiske Studier, Akademisk Forlag.

\section{ELIADE M.}

1951 (rééd. 1983) : Le Chamanisme et les techniques archaïques de l'extase, Paris, Payot.

\section{ELSDON B.}

1925 : «Games and Pastimes of the Maori », Dominion Museum Bulletin, 8, p. 128-147.

\section{Ernout A., Meillet A.}

1967 (4 éd.) : Dictionnaire étymologique de la langue latine, $2^{\mathrm{e}}$ tirage augmenté de corrections nouvelles, Paris, Klincksieck.

\section{Feruglio V., BAFFier D.}

2005 : «Les dessins noirs des salles Hillaire et du Crâne, grotte Chauvet-Pont-d'Arc : chronologie relative ", Bulletin de la Société préhistorique française, 102, 1, p. 149-158.

\section{Fet E., Fet P.}

1941 : Sydvestnorske helleristninger. Rogaland og Lista, Stavanger, Stavanger Museum.

\section{FRANGOIS P.}

2001 : Les Productions céramiques du Chasséen de Villeneuve-Tolosane: évolution stylistique et comparaisons avec les autres faciès chasséens d'Europe occidentale, Toulouse, École des hautes études en sciences sociales, 3 vol.

\section{FREUND G.}

1840: Grand dictionnaire de la langue latine, Paris, Librairie Firmin.

\section{FUGLESTVEDT I.}

1999 : «Adorants, voltigeurs and other mortals : an essay on rock art and the human body ", in GOLDHAHNJ. (DIR.),
Rock Art as Social Representation, EAA, Göteborg 1998, Oxford, Archaeopress (coll. British Archaeological Reports, International Series, 794), p. 25-40.

\section{GALANT P.}

2005: "Sur les pas de J. Abélanet à la grotte du Serrat Nalt et à la Caune de Périllos: nouveaux indices paléospéléologiques en Languedoc-Roussillon ", in MARTZlufF M. (DIR.), Roches ornées, roches dressées, Hommage à Jean Abélanet, Perpignan, Presses Universitaires de Perpignan, Association archéologique des Pyrénées-Orientales, p. 185-197.

\section{GALLES L.}

1873 : «Comment les dolmens pourraient bien avoir été construits par les Gaulois ", Bulletin de la Société polymatique Morbihan, p. 50-55.

\section{Galles R., MAUricet A.}

1864 : Étude sur le Mané-Lud en Locmariaquer, Vannes, Imprimerie Galles.

\section{GARDIN J.-C.}

2000: «Théories générales et méthodes formelles dansl'interprétation de l'art préhistorique », in SHER A. (DIR.), Conference on Prehistoric Art, 1998, Kemerovo, SAPAR Bulletin, p. 77-95.

\section{Giot P.-R., L'HELgOUAC'H J., MONNIER J.} 1998 (1 ${ }^{\text {re }}$ éd. 1979) : Préhistoire de la Bretagne, Rennes, Ouest-France Université, 444 p.

GJESSING G.

1932: Arktistic Halleristninger $i$ Nord-Norge, Oslo, H. Aschehoug.

\section{GoODY J.}

1977 (2e éd. 1979) : La Raison graphique: la domestication de la pensée sauvage (The Domestication of the Salvage Mind), Paris, éd. de Minuit (coll. le Sens commun).

\section{GOSCINY R., UDERzO A.}

1965 : Astérix et Cléopâtre, Paris, Dargaud.

\section{GOULETQUER P.}

1991a: «Les problèmes posés par le "Mésolithique" de Basse-Bretagne: les moyens de les résoudre ", in Mésolithique et Néolithisation, Actes du $113^{e}$ congrès des Sociétés savantes, Strasbourg, 1988, Paris, éd. du CTHS, p. 177-196.

1991b : "Rêves de pierre ", in EVEILLARD J.Y. (DIR.), «La Pierre en Basse-Bretagne : usages et représentations", Cahiers de Bretagne occidentale, 18, p. 128-144.

\section{GOURAUD G.}

2003: Dictionnaire archéologique du pays du vignoble nantais, Nantes (coll. Société nantaise de Préhistoire, 23).

\section{GRANET M.}

1999: La Pensée chinoise, Paris, Albin Michel (coll. Bibliothèque Évolution Humanité).

\section{GRANT C.}

1983 : L’Art rupestre des Indiens de l'Amérique du nord, Capo di Ponte, Jaca Book.

\section{Guerrero Ayuso V. M.}

2004 : "La Marina de la Cerdeña nurágica », Pyrenae, 35, 1, p. 117-155.

\section{Guimbutas M.}

1989 : The Language of the Goddess, London, Thames \& Hudson.

\section{HAARMANN H.}

2005 : «The challenge of the abstract mind : symbols, signs and notational systems in European Prehistory", Documenta Praehistorica, Neolithic Studies, XXXII, 12, p. 221-232.

Hale H. M., Tindale N. B.

1925 : «Observations on Aborigines of the Flinders ranges and records of rock carvings and paintings ", Records of the South Australian Museum, 3 (1), p. 45-60.

\section{HALLENDY N.}

1994: «Inuksuit: semalithic figures constructed by Inuit in the Canadian Arctic ", in MORRISON D., PILONJ.-L. (DIR.), Threads of Arctic Prehistory: Papers in Honour of William E. Taylor J.R., Ottawa, musée canadien des Civilisations (coll. Mercure, Commission archéologique du Canada, 149), p. 385-408.

2001: Inuksuit: the Silent Messengers of the Arctic, Vancouver, Douglas \& McIntyre.

\section{HAMAYON R.}

1990 : La Chasse à l'âme: esquisse d'une théorie du chamanisme sibérien, Nanterre, Société d'Ethnologie.

\section{HENRY M.}

1988 : Voir linvisible : sur Kandinsky, Paris, Bourin.

\section{HOCART A. M.}

1954 (rééd. 2005) : Au Commencement était le rite: de l'origine des sociétés humaines (Social origins), Paris, éd. la Découverte, Mauss.

\section{HÖNEISEN M.}

1985: «Vue d'ensemble sur le Néolithique dans le nord-est de la Suisse ", in Première 
céramique, premier métal: du Néolithique à l'âge du Bronze dans le domaine circum-alpin, Lons-le-Saunier, Musée archéologique, p. 37-68.

\section{HUOT J.-L.}

1994 : Les Premiers villageois de Mésopotamie : du village à la ville, Paris, Armand Collin.

2004: Une archéologie des peuples du ProcheOrient, vol. I, Paris, éd. Errance (coll. Archéologie).

\section{IVANOF I.}

1989 : Le Premier or de l'humanité en Bulgarie, $V^{e}$ millénaire, Catalogue de l'exposition, "Nécropole de Varna », Paris, éd. de la Réunion des Musées nationaux.

1991 : « Das Gräberfeld von Varna : Katalog », in LICHARDUS (DIR.), Die Kupferzeit als historische Epoche, Symposium Saarbrücken und Otzenhausen 6, 1988, vol. 1, Bonn, R. Habelt GMBH, p. 183-208.

JAFFE H. L. C.

1970 : Piet Mondrian, Paris, éd. Cercle d'art (coll. Grands peintres et sculpteurs).

\section{JANNOT J.-R.}

1998: Devins, dieux et démons: regards sur la religion de l'Étrurie antique, Paris, Picard (coll. Antiqua).

JONGE R. M. DE, WAKEFIELD J. S.

2002: How the Sun God Reached America c. 2500 BC: a Guide to Megalithic Sites, Kirkland (USA), MCS Inc.

\section{JOUSSAUME R.}

2003: Les Charpentiers de la pierre: monuments mégalithiques dans le monde, Paris, éd. Maison des roches.

\section{JUSTIN SAINT}

1995 : Apologie pour les chrétiens, éd. et trad., Fribourg (Suisse), Éditions universitaires (coll. Paradosis, 39), VII p. et 151 p.

\section{KADIKOV B.}

2005 : Kollekcia Pietroglifi Altaia, Biysk, Museia V. Bianki.

\section{KALICZ N.}

1970 : Dieux d'argile : l'âge de pierre et de cuivre en Hongrie, Budapest, éd. Corvina (Hereditas).

\section{KARAGEORGHIS V.}

1976 : La Civilisation préhistorique de Chypre, Athènes, Ekdotike Athenon.

KIDDER V., GUERNSEY S. J.

1919 : Archeological Explorations in Northeastern
Arizona, Washington, Government Printing Office, Smithsonian Institution (coll. Bureau of American Ethnology, Bulletin 65)

\section{KINGSLEY S.}

1994: Barbares en Méditerranée, de la Rome tardive au début de l'Islam, Londres, Periplus Publis Ltd.

\section{KNAPPE K.-A.}

1984 : Dürer. Gravures. Euvre complète, Paris, Arts et métiers graphiques.

\section{KÖNIG M.}

1987 : «Les monnaies celtiques, témoignages de temps plus anciens ", in Mélanges J.-B. Colbert de Beaulieu, Paris, éd. le Léopard d'Or, p. 501-507.

\section{KORENEBSKY C. N.}

2001: «Zoomorfie i antropomorfie obrazi iskousstve nlemen MaïkopsckoNovosvobodneïskoï obtsnosti ", in STATEÏ S. (DIR.), Mirobozrenie drevnevo nacelenia Evrasii, Mosckva, Rossiskaya Akademia nauk Inctitut archeologii, p. $45-59$.

\section{KOTCHMAR N. N}

1995 : Korreliatsia naskalnikh isobrajenii iakouti $i$ tchoukotki, Kemerovo, Nackalnoie Iskosstva Asii, Vitousk 1, p. 43-45.

\section{KUBAREV V. D.}

1988 : Drebinie rospissi karakola, Novossibirsk, Nauka cibirskoie otdepenie.

2002: "Traces of shamanic motives in the petroglyphs and burial paintings of the Gorno-Altaï », in RoZWADOWSKI A., Kosko M. M. (DIR.), Spirits and Stones : Shamanism and Rock Art in Central Asia and Siberia, Poznan, Institute Wschodni, p. 99-119.

\section{KULUNDİ Z .}

1951 : Knjiga o knjizi, t. I, Historija pisama, Zagreb, éd. Kolska knjiga.

\section{LACROIX L.}

1938 (rééd. 1968 et 1997) : Les Derniers baleiniers français, Rennes, Ouest-France.

\section{LAPORTE L., LE ROUX C.-T.}

2004 : Bâtisseurs du Néolithique: mégalithismes de la France de l'Ouest, Paris, éd. Maison des Roches.

LAPORTE L., PICQ C. (DIR.)

2002: «Les occupations néolithiques du vallon des Ouchettes (Plassay, CharenteMaritime) », Gallia Préhistoire, 44, p. 1-120.
LE BOLlOCH M., DUbouloz J.,

PlateaU M.

1986 : «Les sépultures rubanées et l'enceinte de la fin du Ve millénaire ", Revue archéologique de Picardie, 1/2, p. 3-12.

\section{LE COUÉDIC D.}

1998: «Le paysage tué par ceux-là mêmes qui l'adoraient », Kreiz, 11, p. 7-20.

\section{LE GOFF J.}

1985 : L'Imaginaire médiéval, Paris, Gallimard (coll. Biblio. des Histoires).

\section{LEISNER G., LEISNER V.}

1956: Die Megalithgräber der Iberischen Halbinsel: der Westen, vol. 1, Berlin, Walter de Gruyter \& Co (coll. RömischGermanische Forschungen).

\section{LEROI-GOURHAN A.}

1964: Le Geste et la parole -I- Technique et langage, Paris, Albin Michel.

\section{Leroi-Gourhan A., Allain J.}

1979: Lascaux inconnu (Dordogne), Paris, CNRS Éditions (coll. Suppl. à Gallia Préhistoire, XII).

\section{LE ROUX C.-T.}

1992 : «Cornes de pierre... », in Paysans et bâtisseurs : l'émergence du Néolithique atlantique et les origines du Mégalithisme, Actes du XVII colloque interrégional sur le Néolithique, Vannes, 29-31 oct. 1990, Rennes (coll. Suppl. à la Revue archéologique de l'Ouest, 5), p. 237-244.

1997 : «Et voguent les menhirs? », Association Manche Atlantique pour la recherche archéologique dans les îles (AMARAI), 10, p. 5-18.

2001 : Carnac, Locmariaqueret Gavrinis, Rennes, Ouest-France (coll. Patrimoine).

\section{LE ROUZIC Z.}

1912: Carnac: fouilles faites dans la région. Dolmen à galerie et grand dallage $d u$ Mané Lud, Vannes, Imprimerie Galles, p. $225-232$.

1913 : Carnac: menhirs-statues avec signes figuratifs et amulettes ou idoles des dolmens $d u$ Morbihan, Nantes, Imprimerie Duca.

\section{LETTERLE F.}

1992 : «Quelques réflexions à propos de la chronologie du Néolithique moyen d'Armorique ", in Paysans et bâtisseurs : l'émergence du Néolithique atlantique et les origines du Mégalithisme, Actes du XVII colloque interrégional sur le Néolithique, Vannes, 29-31 oct. 1990, Rennes (coll. Suppl. à la Revue archéologique de l'Ouest, 5), p. 177-193. 


\section{LÉVI-STRAUSS C.}

1962 (rééd. 1990) : La Pensée sauvage, Paris, Plon (coll. Agora).

LÉVY A.

2003: Les Machines à faire-croire-1- Formes et fonctionnements de la spatialité religieuse, Paris, éd. Anthropos (coll. la Bibliothèque des formes).

\section{L'HELGOUAC'H J.}

1965 : Les Sépultures mégalithiques en Armorique, Rennes, Université de Rennes.

1991: «Déesses et figurations cornues du Néolithique », in LumleyH. DE, Lautman F. (DIR.), Le Mont Bego: une montagne sacrée de l'âge du Bronze, Paris, Institut de paléontologie humaine, p. 537- 557.

1994 : Locmariaquer, Paris, Gisserot.

1998: "Navigation et navires durant la période néolithique en Bretagne: sur l'interprétation des gravures mégalithiques ", in Actes du $120^{\circ}$ congrès national des Sociétés savantes, Aix-en-Provence, 1995, Paris, éd. du CTHS, p. 151-161.

L'HELGOUAC'H J., LECORNEC J.

1976: «Le site mégalithique Min Goh Ru près de Larcuste à Colpo (Morbihan)", Bulletin de la Société préhistorique française, 73, p. 370-397.

\section{LE LIVRE DES MORTS}

1976: La Mythologie égyptienne de l'au-delà, Paris, Le Cerf.

\section{LUCENA MARTIN A.-M.}

1980 : «La escasa representación de la cruz latina en el primer arte cristano ", Revista de arqueologia, 218, p. 38-46.

LUMLEY H. DE, LAUTMAN F. (DIR.)

1991 : Le Mont Bego : une montagne sacrée de l'âge du Bronze, Paris, Institut de paléontologie humaine.

\section{LUQUET G.-H.}

1910 : «Sur la signification des pétroglyphes des mégalithes bretons ", Revue de l'École d'anthropologie de Paris, 10, p. 224-253.

\section{MAERTENS J.-T}

1978: Ritologiques 4. Dans la peau de autres, Paris, Aubier-Montaigne.

\section{MAHÉ C.}

1825 : Essai sur les Antiquités du département du Morbihan, Vannes, Imprimerie Galles.

MAIANI M., QUER S.

1996 : Voyage vers l'île mystérieuse : de la Polynésie à l'île de Pâques, Milan, Venise, ed. A. Pizzi, Erizzo Editrice.

\section{MAISONNEUVE B.}

1983: Les Signaux gravés dans les tombes à couloir en Armorique, mémoire de Maîtrise, Université Paris-I.

\section{MALEVITCH K. S.}

1920 (éd. 1974) : Écrits I : de Cézanne au suprématisme, Lausanne, éd. l'Âge d'homme.

\section{MANTU C. M.}

1998: Cultura Cucuteni: evolutie, cronologie, legaturi, Piatra-Neamt, Muzee de Istorie (coll. Bibliotheca Memoriae Antiquitatis, V).

\section{MARCHAND G., TRESSET A.}

2004: «Derniers chasseurs-cueilleurs et premiers agriculteurs sur la façade atlantique de l'Europe ", in GUILAINE J. (DIR.), Aux marges des grands foyers du Néolithique: périphéries débitrices ou créatrices?, Paris, éd. Errance, p. 257-280.

\section{MARGUERON J.}

1982: Recherches sur les palais mésopotamiens de l'âge du Bronze, I et II, Paris, Paul Geuthner.

\section{MARIN L.}

1993 : Des pouvoirs de limage, Paris, Le Seuil (coll. l'Ordre philosophique).

\section{MARKALE J.}

1987: Carnac et l'énigme de l'Atlantide, Paris, Pygmalion, G. Watelet.

\section{MARTINS A.}

2006: "Gravuras rupestres do Noroeste peninsular : a Chã da Rapada ", Revista portuguesa de arqueologia, 9, 1, p. 47-70.

\section{MARTI Oliver B.}

1990 : «Impressed Cardial decoration and rock shelter art in eastern Spain ", in CAHEN D., OTTE M. (DIR.), Rubané et Cardial, Liège (coll. ERAUL, 39), p. 405415 .

\section{MARTYINOV A.}

2001: Tomskaya Pisanitsa, Kemerovo, OKY Izdatelstva « Letopiss ».

MASSON V. M., MERPERT N. Y.,

MUNCHAEIV R. M., TCHERNICH E. K.

1982 : Eneolit SSSR-II-Eneolit Kavkaza, Moskva, Izdatelstva Nauka.

MAYSMOR B.

2001: The Maori Te Manu Tukutuku,
Wellington, Nouvelle-Zélande, Steele Roberts.

\section{MCLEAN W.}

1970: Contribution à l'étude de l'iconographie populaire de l'érotisme, Paris, Maisonneuve et Larose.

\section{MEDVEDEV V. E.}

2001 : «Sources of some sculptural and rock images in Prehistoric art of the southern Far East and finds of the Osipovka culture on the Amur river ", Eurasia, 4 (8), p. 77-94.

\section{MELlaART J.}

1967 : Catal Hüyük : a Neolithic Town in Anatolia, London, Thames \& Hudson.

\section{MELLER H.}

2002: «Die Himmelsscheibe von Nebra: ein frübronzezeitlicher Fund von aussergewöhnlicher Bedeutung ", Archäologie in Sachsen-Anhalt, 1, p. 7-20.

MENS E.

2002: L'Affleurement partagé: gestion du matériau mégalithique et chronologie de ses représentations gravées dans le Néolithique moyen armoricain, thèse de Doctorat nouveau régime, Université de Nantes, 3 vol.

2003: «Gravures rupestres nouvellement découvertes à Batz-sur-Mer ", Cahiers du pays de Guérande, 43, p. 56-60.

\section{MERLEAU-PONTY M.}

1945 (rééd. 1976) : Phénoménologie de la perception, Paris, Gallimard.

1964 (rééd. 1985) : L'Eil et l'esprit, Paris, Gallimard (coll. Folio Essais).

\section{MÉroc L., Simmonet G.}

1979 : «Les sépultures chasséennes de SaintMichel-du-Touch (Haute-Garonne) ", Bulletin de la Société préhistorique française, 76, 10-12, p. 379-403.

\section{MILIN G.}

1989: «La traversée prodigieuse dans le folklore et l'hagiographie celtiques : essai de typologie", in "Saints et moines : du mythe à l'histoire", Bulletin de la Société archéologique du Finistère, 118, p. 125-140.

1991: «La traversée prodigieuse dans le folklore et l'hagiographie celtiques: de la merveille au miracle », Annales de Bretagne, 98, p. 1-25.

\section{MODDERMAN P. J. R.}

1985 : «Die Bandkeramik im Graetheidegebiet, Niederländisch- 
Limburg ", Bericht römisch-germanischen Kommission, 66, p. 25-121.

\section{MoravetTi A., TOzzi C.}

1995 : Guide archeologiche. Preistoria e Protostoria in Italia : Sardegna, UISPP, Forli, ABACO ed.

\section{MORDANT D.}

1991 : «Le site des Réaudins à Balloy (Seineet-Marne) : premiers résultats », in Actes du $X V^{e}$ colloque interrégional sur le Néolithique, Châlons-sur-Marne, 1988, Châlons-surMarne, Association régionale pour la protection et l'étude du patrimoine préhistorique, p. 33-43.

MORTILlet A. DE

1894: «Les figures sculptées sur les monuments mégalithiques de France ", Revue de l'École d'anthropologie de Paris, 9, p. 273-307.

\section{MORTILleT G. DE}

1866 : Le Signe de la croix avant le christianisme, Paris, Librairie E. Reinwald

MOSER M., DORFER L., SPINDLER K., BAHR F., EGARTER-VIGL E., DOHR G., KENNER T.

1999: «Are Ötzi's tattoos acupuncture? Skin markings on the Tyrolean Iceman may have been treatment for his ills ", Discovering Archaeology, 1 (1), p. 16-17.

\section{MÜLLER-KARPE $\mathbf{H}$.}

1968: Handbuch der Vorgeschichte: Jungsteinzeit, 2, München, Beck'sche Verlagsbuchhandlung.

MUlVin L., Sidebotham S. E.

2004 : « Roman game boards from Abu Sha'ar (Red Sea cost, Egypt) ", Antiquity, 78, 301, p. 602-617.

\section{NAKAMURA $\mathbf{S}$.}

2005 : « Le riz, le jade et la ville : évolution des sociétés néolithiques», Annales, Histoire, Sciences sociales, 60 (5), p. 975-1 068.

\section{OCETROV E.}

1985 : Anciens Rus vivants (en russe), Moscou, éd. Procbechienie.

OChibKInA S. V. (DIR.) avec la collab. de TITOV V. S., SIDOROV V. V.

1996: Arkeologia. Neolit Severnoï Eurazii, Moskva, Nauka.

\section{OKLADNIKOVA E. A.}

1996: "Shaman I Vselennaia: istoki shamanisticheskikh predstavlenij (po mate- rialam naskal ; nogo iskusstva Sibiri)", Kur'er Petrovskoj Kunstkamery, 4-5, SanktPetersburg, Muzej antropologii i etnografii im. Petra Velikogo (Kunstkamera), p. $90-114$.

\section{PÉQuart M., PÉquart ST-J.}

1954: Hoëdic: deuxième station-nécropole du Mésolithique côtier armoricain, Anvers, De Sikkel.

PéQuart M., PéQuart ST-J., Le Rouzic Z. 1927 : Corpus des signes gravés des monuments mégalithiques du Morbihan, Paris, Picard.

\section{Pétrequin P., Pétrequin A.-M.}

1993: Écologie d'un outil: la hache de pierre en Irian Jaya (Indonésie), Paris, CNRS Éditions (coll. Monographies du CRA, 12)

Petru S.

À paraître : « Symbolism of the non-figurative signs on the portable art from the Late Palaeolithic site Zemono ", in Symbols and Symbolism, $11^{\text {th }}$ Neolithic Seminar, Ljubljana (Slovénie) 4-7 nov. 2004 (coll. Documenta praehistorica, XXXII).

PICARD C.

1976 : « Les représentations du sacrifice molk sur les stèles de Carthage ", Karthago, XVII, 1973-1974, p. 67-138.

PIEL T.

2000 : «Le dieu, le roi et le prêtre : le bâton augural dans les représentations étruscolatines du pouvoir ", in Éléments d'architecture (exploration d'un tertre funéraire à Lannec er Gadouer, Erdeven, Morbihan. Constructions et reconstructions dans le Néolithique morbihannais. Propositions pour une lecture symbolique), Chauvigny, éd. chauvinoises (coll. Mémoire, 19), p. 689-711.

2003 : « Réflexions sur la nature du pouvoir en Italie centrale : les cités étrusques et la cité romaine ( $\mathrm{VII}^{\mathrm{e}}-\mathrm{III}{ }^{\mathrm{e}} \mathrm{s}$. av. J.-C.) ", in Idéologies et valeurs dans le monde romain, Hommage à C. Lepelley, Paris, Picard, p. 23-35.

\section{Platon}

1967 : Protagoras, Paris, Flammarion.

1992 : Timée Critias, Paris, Flammarion.

\section{Plutaroue}

1999: Euvres, Paris, éd. Arlea.

\section{PRIUli A.}

1979: Preistoria in Vallecamonica, Capo di Ponte, Museo didattico d'Arte e Vita Preistorica.

\section{Proust M.}

1920 (rééd. 1988) : Le Côté de Guermantes, Paris, Gallimard (coll. Folio classique).

\section{PULLIGNY VICOMTE DE}

1879 : L'Art préhistorique dans l'ouest et notamment en Haute-Normandie, Évreux, Imprimerie Ch. Hérissey.

\section{Purcell A.}

2002: « Excavation of three neolithic houses at Corbally, Kilcullen, Co. Kildare », The Journal of Irish Archaeology, XI, p. 31-75.

RACZKY P. (DIR.)

1987: The Late Neolithic in the Tisza Basin, Budapest, Szolnok, Kassuth Press.

\section{RADKOWSKI G.-H. DE}

2002: Anthropologie de l'habiter: vers le nomadisme, Paris, PUF.

\section{RAJNOVICH G.}

1994 : Reading Rock Art : Interpreting the Indian Rock Paintings of the Canadian Shield, Toronto, Natural Heritage/Natural History Inc.

\section{REINACH S.}

1893 : « Le mirage oriental », L'Anthropologie, 4, p. $539-578$ et p. $698-732$.

\section{ROJO GUERRA M., KUNST M.}

1999: «La lampara y la pena de la abuela: una propuesta secuencial para el Neolitico interior en el ambito funerario », II Congrès del Neolitic a la Peninsula ibèrica, Valancia, Universidad de Valencia, p. 503-512.

\section{SANDARS N.}

1985 (2e éd.) : Prehistoric Art in Europe, London, Penguin.

\section{SANG MOG LEE}

2003: Les Gravures rupestres du site de dans la partie méridionale de la péninsule coréenne, Paris, thèse de Doctorat du Muséum national d'histoire naturelle.

\section{SANSONi U., MARRETTA A.}

2002 : « Recent discoveries in Zurla and Dos Cuì ", Adoranten, 9, p. 5-14.

\section{SANTos-estévez M., SeOANe-Veiga Y.}

2005 : «Escavacion no contorno dun petroglifo en a ferrarura (Ouenese-Galiza) », Arkeos, 15 (2004), p. 37-53.

\section{SCHOTT S.}

1953 : «Symbol und Zauber also Grundform altñgyptischen Denkens", Studium Generale, 65, p. 278-288. 
SCHUMANN-ANTELME R., RosSINI S.

2003 : Dictionnaire illustré des dieux de l'Égypte, Paris, éd. du Rocher.

\section{SEgLIE D., RicGHIARdi P., CINQUETTI M.}

1991: «L'arte rupestre nelle Alpi del Piemonte: temi, problemi e rapporti col Monte Bego, santurio dell'eta del Bronzo ", in LUMLEY H. DE, LAUTMAN F. (DIR.), Le Mont Bego : une montagne sacrée de l'âge du Bronze, Paris, Institut de paléontologie humaine, p. 195-245.

\section{SeTtegast M.}

1986 (rééd. 1990) : Plato Prehistorian: 10,000 to 5,000 B.C. : Myth, Religion, Archaeology, New York, Lindisfarne Press, Copyrighted Material.

SHEE TWOHIG E.

1981: The Megalithic Art of Western Europe, Oxford, Clarendon Press, $560 \mathrm{p}$.

2002 : « Context and chronology of the carved stone at Clofinlough, County Offaly ", Journal of Royal Society of Antiquaries of Ireland, 132, p. 99-113.

Sirbu V., Pandrea S.

2003: "Signs on vessel buttoms from the developed Neolithic in the CarpathianBalkan region ", in NIKOLOVA L. (DIR.), Early Symbolic Systems for Communication in Southeast Europe, Oxford, Archaeopress (coll. British Archaeological Reports, International Series, 1139), p. 193-202.

\section{SMIRNOV A. M.}

2001: «Izobrajenia hramovih structur i ciujetov v evpopeïkom megalititcheskoï iskusstve V-IV-III ticiatcheletiyah do NE : opit identifikatsii ", in STATEÏ S. (DIR.), Mirobozrenie drevnevo nacelenia Eurasii, Moskva, Rossiskaya Akademia nauk Institut archeologii, p. 60-89.

2004: «Izobrazhenija posokhov na antropomorfnykh izvayanyakh epokhi eneolita $\mathrm{v}$ severnom pritchernomorye i Sredisemhomorye. Analogii, interpretatsii ", in Pamiatniki arkeologii $i$ drevnego isckustva evrasii, Moskva, Rossiskaya akademia nauk institut archeologii, p. 65-103.

\section{SPROCKHOFF E.}

1938: Die Nordische Megalithkultur, Berlin, Leipzig, Walter \& Gruyter.
TANDA G.

1979: "Arte e religione in Sardegna ", in Symposium III, Prehistoric Art and Religion, the Intellectual Expressions of Prehistoric Man: Art and Religion, Valcamonica, 1979, Capo Ponte, Centro camuno di Studi Preistorici, p. 261-279.

\section{THEVENOT J.-P.}

1969 : «Éléments chasséens de la céramique de Chassey ", Revue archéologique de l'Est et du Centre-Est, XX, 1-2, p. 7-95.

\section{TOBIN V. A.}

1989: Theological Principles of Egyptian Religion, New York, Peter Lang, American University Studies, series 7 (coll. Theology and Religion, 59).

\section{TODOROVA H.}

1978 : The Eneolithic Period in Bulgaria in the Fifth Millennium B.C., Oxford, Archaeopress (coll. British Archaeological Reports, Supplementary Series, 49).

\section{TORBRÜGGE $\mathbf{W}$.}

1979: Die Hallstattzeit in der Oberpfalz. Materialhefte zur bayerischen Vorgeschichte, Reihe A, Band 39 (Kallmünz, Opf.).

\section{TRESSET A.}

2002 : «L'apparition de l'élevage à la marge nord-ouest de l'Europe: un processus complexe et diversifié », Annales de la Fondation Fyssen, 17, p. 43-54.

2005: «La place changeante des bovins dans les bestiaires du Mésolithique final et du Néolithique d'Armorique et des régions limitrophes ", in MARCHAND G., TRESSET A. (DIR.), Unité et diversité des processus de néolithisation sur la façade atlantique de l'Europe (6 ${ }^{e}$ 4 millénaires avant J.-C.), Table ronde de Nantes, 26-27 avril 2002, Paris, Société préhistorique française (coll. Mémoire, 36), p. 273-286.

\section{TZAFERIS V.}

1985: "Crucifixion: the archaeological evidence ", Biblical Archaeology Review, 9, p. 44-53.

VAN GENNEP A.

1909 : Les Rites de passage, Paris, Picard.

VAQUER J.

1975 : La Céramique chasséenne du Languedoc,
Carcassonne, Laboratoire de Préhistoire et de Palethnologie, 368 p.

\section{VAQUERO LASTRES J.-L.}

1999: La Configuration de l'espace dans les sociétés ayant bâti des tertres funéraires dans le nord-ouest Ibérique, Oxford, Archaeopress (coll. British Archaeological Reports, International Series, 821).

\section{VARELA GOMES M.}

1997a : "Cromelque de Portela de Mogos ", in Paisagens arqueologicas aoeste de Evora, Evora, Câmara municipal de Evora, p. 35-39.

1997b : «Estátuas-menires antropomórficas do Alto-Alentejo : descobertas recentes e problematica ", Brigantium, 10, p. 255-279.

À paraître: "Time and signs: southern Portugal megalithic art diachrony ", in $15^{\text {th }}$ UISPP Congress, Lisboa (Portugal), 2006 (coll. British Archaeological Reports).

\section{VAZEILLES D.}

1995: «Pictographes et écriture des Indiens et des Inuit: des époques préhistoriques jusqu'au XXe siècle", in Rencontres avec les Indiens d'Amérique du Nord, Études rassemblées par D. Vazeilles, Montpellier, Imprimerie de recherche, Université Montpellier-III (coll. LASPEC), p. 83-104.

\section{VERNEAU R.}

1949 : Histoire naturelle illustrée: l'homme, races et coutumes, Paris, librairie Larousse.

\section{VOLTAIRE}

1764 (rééd. 1964) : Dictionnaire philosophique, Paris, Garnier-Flammarion.

\section{VORAGINE J. DE}

1255 (rééd. 1967) : La Légende dorée, Paris, Garnier-Flammarion.

\section{ZÁPÓTOCKY M.}

1991: «Frühe Streitaxtkulturen im mitteleuropaïschen Aneolithikum », in LICHARDUS M. (DIR.), Die Kupferzeit als historische Epoche, Symposium Saarbrücken und Otzenhausen 6, 1988, vol. 1, Bonn, R. Habelt GMBH, p. 465-475.

\section{ZILHÃO J.}

1998 : « A Passagem do Mesolitico ao Neolitico na costa do Alentejo ", Revista portuguese de arqueologia, 1 (1), p. 27-44. 